\title{
A Chemical Composition Survey of the Iron-complex Globular Cluster NGC 6273 (M19)
}

\author{
Christian I. Johnson ${ }^{1,8}$, Nelson Caldwell ${ }^{1}$, R. Michael Rich ${ }^{2}$, Mario Mateo ${ }^{3}$, John I. Bailey, III $^{4}$, \\ William I. Clarkson ${ }^{5}$, Edward W. Olszewski ${ }^{6}$, and Matthew G. Walker ${ }^{7}$ \\ ${ }^{1}$ Harvard-Smithsonian Center for Astrophysics, 60 Garden Street, MS-15, Cambridge, MA 02138, USA; cjohnson@cfa.harvard.edu, ncaldwell@cfa.harvard.edu \\ ${ }^{2}$ Department of Physics and Astronomy, UCLA, 430 Portola Plaza, Box 951547, Los Angeles, CA 90095-1547, USA; rmr@astro.ucla.edu \\ ${ }^{3}$ Department of Astronomy, University of Michigan, Ann Arbor, MI 48109, USA; mmateo@umich.edu \\ ${ }^{4}$ Leiden Observatory, Leiden University, P.O. Box 9513, 2300RA Leiden, The Netherlands; baileyji@strw.leidenuniv.nl \\ ${ }^{5}$ Department of Natural Sciences, University of Michigan-Dearborn, 4901 Evergreen Road, Dearborn, MI 48128, USA; wiclarks@umich.edu \\ ${ }^{6}$ Steward Observatory, The University of Arizona, 933 N. Cherry Avenue, Tucson, AZ 85721, USA; eolszewski@as.arizona.edu \\ 7 McWilliams Center for Cosmology, Department of Physics, Carnegie Mellon University, \\ 5000 Forbes Avenue, Pittsburgh, PA 15213, USA; mgwalker@andrew.cmu.edu \\ Received 2016 August 23; revised 2016 October 17; accepted 2016 October 20; published 2017 February 17
}

\begin{abstract}
Recent observations have shown that a growing number of the most massive Galactic globular clusters contain multiple populations of stars with different $[\mathrm{Fe} / \mathrm{H}]$ and neutron-capture element abundances. NGC 6273 has only recently been recognized as a member of this "iron-complex" cluster class, and we provide here a chemical and kinematic analysis of $>300$ red giant branch and asymptotic giant branch member stars using high-resolution spectra obtained with the Magellan-M2FS and VLT-FLAMES instruments. Multiple lines of evidence indicate that NGC 6273 possesses an intrinsic metallicity spread that ranges from about $[\mathrm{Fe} / \mathrm{H}]=-2$ to -1 dex, and may include at least three populations with different $[\mathrm{Fe} / \mathrm{H}]$ values. The three populations identified here contain separate first ( $\mathrm{Na} / \mathrm{Al}$-poor) and second ( $\mathrm{Na} / \mathrm{Al}$-rich) generation stars, but a $\mathrm{Mg}-\mathrm{Al}$ anti-correlation may only be present in stars with $[\mathrm{Fe} / \mathrm{H}] \gtrsim-1.65$. The strong correlation between $[\mathrm{La} / \mathrm{Eu}]$ and $[\mathrm{Fe} / \mathrm{H}]$ suggests that the sprocess must have dominated the heavy element enrichment at higher metallicities. A small group of stars with low $[\alpha / \mathrm{Fe}]$ is identified and may have been accreted from a former surrounding field star population. The cluster's large abundance variations are coupled with a complex, extended, and multimodal blue horizontal branch (HB). The HB morphology and chemical abundances suggest that NGC 6273 may have an origin that is similar to $\omega$ Cen and M54.
\end{abstract}

Key words: globular clusters: general - globular clusters: individual (NGC 6273, M19) - stars: abundances

Supporting material: machine-readable tables

\section{Introduction}

Galactic globular clusters are no longer considered pure simple stellar populations. Although large and often (anti-) correlated star-to-star light element abundance variations have long been known to exist within individual globular clusters (e.g., Cohen 1978; Peterson 1980; Cottrell \& Da Costa 1981; Sneden et al. 1991; Pilachowski et al. 1996b; Kraft et al. 1997; Shetrone \& Keane 2000; Gratton et al. 2001; Ivans et al. 2001), the ubiquitous nature of their peculiar chemical compositions has only recently been recognized. Large sample spectroscopic surveys have revealed that all but perhaps the lowest mass clusters (Walker et al. 2011; Villanova et al. 2013; Salinas \& Strader 2015) exhibit similar, but not identical, (anti-) correlations among elements ranging from carbon to aluminum (e.g., Carretta et al. 2009b, 2009c; Mészáros et al. 2015). In many cases, He enhancements coincide with increased abundances of $\mathrm{N}, \mathrm{Na}$, and $\mathrm{Al}$ and decreased abundances of C, O, and Mg (e.g., Bragaglia et al. 2010a, 2010b; Dupree et al. 2011; Pasquini et al. 2011; Villanova et al. 2012; Marino et al. 2014a; Mucciarelli et al. 2014). Except for CN variations due

\footnotetext{
* Based on observations made with the NASA/ESA Hubble Space Telescope, obtained at the Space Telescope Science Institute, which is operated by the Association of Universities for Research in Astronomy, Inc., under NASA contract NAS 5-26555. These observations are associated with program GO14197. This paper includes data gathered with the $6.5 \mathrm{~m}$ Magellan Telescopes located as Las Campanas Observatory, Chile.

${ }^{8}$ Clay Fellow.
}

to in situ mixing, these interconnected light element abundance patterns may be unique to old ( $\gtrsim 6$ Gyr) globular cluster environments (e.g., Pilachowski et al. 1996a; Sneden et al. 2004; Mucciarelli et al. 2008; Bragaglia et al. 2014).

Large light element abundance variations can have a significant effect on a star's structure and spectrum (e.g., see Piotto et al. 2015; their Figure 1), and recent near-UV observations from the Hubble Space Telescope (HST) have exploited this property to reveal a further connection between chemical compositions and globular cluster formation. A key observational constraint for globular cluster formation scenarios is whether the range of light element abundances follows a continuous distribution or falls into discrete groups. Although some purely spectroscopic evidence supports clusters hosting discrete groups with unique light element chemistry (e.g., Carretta et al. 2009c, 2014; Johnson \& Pilachowski 2010; Carretta 2014, 2015; Cordero et al. 2014; Roederer \& Thompson 2015), HST photometry has been particularly efficient at showing that most or all Galactic globular clusters host multiple distinct populations rather than continuous distributions (e.g., Piotto et al. 2007, 2015; Bragaglia et al. 2010b; Milone et al. 2013, 2015a, 2015b; Marino et al. 2016). The combined data from spectroscopy and photometry provide strong evidence that globular clusters experienced multiple rounds of star formation. However, the detailed processes by which globular clusters form, and the nucleosynthetic origins of the light element abundance variations, remain unresolved issues (e.g., see recent discussions in Valcarce \& Catelan 2011; 
Bastian et al. 2015; Bastian \& Lardo 2015; Renzini et al. 2015; D'Antona et al. 2016)

Despite most globular clusters exhibiting large light element abundance variations, most systems do not display the same complexity for the heavier elements. The $[\mathrm{Fe} / \mathrm{H}]^{9}$ and $[\mathrm{X} / \mathrm{Fe}]$ ratios for most $\alpha$ and Fe-peak elements vary by $\sim 0.1$ dex or less within an individual cluster (e.g., Carretta et al. 2009a), but intrinsic variations at the few percent level may be present for all elements (Yong et al. 2013). Some clusters exhibit primordial abundance variations for elements produced by the rapid neutron-capture process (r-process), but many do not (e.g., Roederer 2011). Most clusters also fail to show chemical signatures of extended star-formation histories, such as elevated slow neutron-capture (s-process) abundances or low $[\alpha / \mathrm{Fe}]$ ratios. More metal-rich clusters tend to exhibit stronger s-process signatures (e.g., higher average $[\mathrm{Ba} / \mathrm{Eu}]$ or $[\mathrm{La} / \mathrm{Eu}]$ ratios) than their more metalpoor counterparts (e.g., Simmerer et al. 2003; Gratton et al. 2004; James et al. 2004; Cohen \& Meléndez 2005; Carretta et al. 2007; D'Orazi et al. 2010; Worley \& Cottrell 2010), but these differences are likely driven by the broader chemical enrichment of the Galaxy.

Interestingly, a growing number of clusters have been discovered that exhibit chemical and morphological characteristics consistent with extended star-formation histories, and may represent a new class of objects. These "iron-complex"10 clusters are characterized as having: (1) broadened or multimodal $[\mathrm{Fe} / \mathrm{H}]$ distribution functions with dispersions exceeding $\sim 0.1$ dex when measured using high-resolution spectra $^{11}$; (2) complex color-magnitude diagrams and split red giant branch (RGB) sequences when observed with $h k$ narrow-band photometry (e.g., Lee 2015; Lim et al. 2015); (3) and correlated abundances of $[\mathrm{Fe} / \mathrm{H}]$ and elements likely produced by the main s-process (e.g., Ba and La). To date, $\sim 10$ iron-complex clusters have been discovered (e.g., see Da Costa 2016a, their Table 1; Marino et al. 2015, their Table 10). ${ }^{12}$ Many of these systems also have about the same metallicity $([\mathrm{Fe} / \mathrm{H}] \sim-1.7)$, have very blue and extended horizontal branch (HB) morphologies, and are among the most massive clusters in the Galaxy $\left(M_{\mathrm{V}} \lesssim-8\right)$. The iron-complex cluster M54 may be the nuclear star cluster of the Sagittarius dwarf galaxy (e.g., Bellazzini et al. 2008), and the most massive ironcomplex cluster omega Centauri ( $\omega$ Cen) is strongly suspected to be a stripped dwarf galaxy nucleus as well (e.g., Bekki \& Freeman 2003). Similarly, the iron-complex clusters NGC 1851 and M2 may also be the stripped cores of former dwarf galaxies (e.g., Olszewski et al. 2009; Kuzma et al. 2016). Therefore, iron-complex clusters may be the relics of more massive systems, the remnants of previous Milky Way

\footnotetext{
$9[\mathrm{~A} / \mathrm{B}] \equiv \log \left(N_{\mathrm{A}} / N_{\mathrm{B}}\right)_{\mathrm{star}}-\log \left(N_{\mathrm{A}} / N_{\mathrm{B}}\right)_{\odot}$ and $\log \epsilon(\mathrm{A}) \equiv \log \left(N_{\mathrm{A}} / N_{\mathrm{H}}\right)+12.0$ for elements $\mathrm{A}$ and $\mathrm{B}$.

${ }^{10}$ Note that iron-complex clusters are the same as the "anomalous" and "s-Feanomalous" clusters discussed in Marino et al. (2015). As mentioned in Johnson et al. (2015b), we prefer to avoid using the word "anomalous" in this context because the word has multiple historical definitions. Additionally, the anomalous label may not be appropriate if additional systems continue to be found.

${ }^{11}$ Note that the metallicity dispersions are contested for some clusters (Mucciarelli et al. 2014; Lardo et al. 2016; but see also Lee 2016).

${ }^{12}$ Terzan 5 is not included in the aforementioned lists but has also been shown to contain multiple generations of stars with distinct chemical compositions (Ferraro et al. 2009; Origlia et al. 2011, 2013; Massari et al. 2014).
}

accretion events, and/or trace a particular time or accretion period in the Galaxy's formation history.

Among the iron-complex cluster class, $\omega$ Cen, M54 and the Sagittarius system, M2, NGC 5286, and NGC 6273 (M19) stand out as particularly interesting. These clusters exhibit broad metallicity distributions with discrete populations occurring near the same $[\mathrm{Fe} / \mathrm{H}]$ values, and also host trace populations of metal-rich stars with peculiar chemical compositions (e.g., Pancino et al. 2002; Carretta et al. 2010a; Johnson \& Pilachowski 2010; Marino et al. 2011a, 2015; McWilliam et al. 2013; Yong et al. 2014; Johnson et al. $2015 b$ ). In order to investigate this phenomenon further, we have obtained high-resolution spectra of $>800 \mathrm{RGB}$ and asymptotic giant branch (AGB) stars located near the massive bulge cluster NGC 6273. Following Johnson et al. (2015b), Han et al. (2015), and Yong et al. (2016), we aim to investigate the cluster's metallicity distribution function and trace the cluster's detailed chemical composition across its various stellar populations.

\section{Observations and Data Reduction}

\subsection{Magellan Spectroscopic Data}

In Johnson et al. (2015b), we identified an intrinsic metallicity spread in NGC 6273, and noted the existence of several stars redder than the formal RGB that could belong to an even more metal-rich component. Since the previous observations were restricted to the color range $0.7 \leqslant J-K_{\mathrm{S}} \leqslant$ 1.0 on the upper RGB, we expanded the target selection criteria for the new observations to include stars in the color range of $0.6 \leqslant J-K_{\mathrm{S}} \leqslant 1.3$. The new observations also span luminosities from the HB to the RGB-tip, and range from 0.53 to 13 '.98 in projected distance from the cluster center (see Figure 1). However, stars closer to the cluster center were given higher priorities in the target ranking process. All coordinates and photometry for the target selection process were taken from the Two Micron All Sky Survey (2MASS; Skrutskie et al. 2006) database.

In order to efficiently obtain a large number of highresolution spectra, we employed the Michigan/Magellan Fiber System (M2FS; Mateo et al. 2012) and MSpec multiobject spectrograph mounted on the Magellan-Clay $6.5 \mathrm{~m}$ telescope. In single order mode, M2FS is capable of placing 2561 !'2 fibers on targets across a nearly $30^{\prime}$ field of view. However, additional orders can be observed simultaneously using a cross-disperser, at the expense of fewer targets. We utilized both options for this project. The first setup operated in single order mode and was optimized to observe the 8542 and $8662 \AA$ near-infrared Calcium II Triplet (CaT) lines. These data provided radial velocities and $\mathrm{CaT}$ metallicities for 466 stars, and permitted an investigation into the full spatial, color, and metallicity extent of NGC 6273. The second setup ("Bulge_GC1" filter) included 6 consecutive orders, spanned $6120-6720 \AA$, allowed for up to 48 fibers to be allocated per configuration, and was used to obtain radial velocities and detailed chemical abundances for 82 stars. As can be seen in Figure 1, both data sets spanned broad color and radial distance ranges, but the $\mathrm{CaT}$ data extended to fainter stars.

Both instrument setups utilized a four amplifier slow readout mode and were binned $2 \times 1$ (spatial $\times$ dispersion). The $\mathrm{CaT}$ and Bulge_GC1 observations were taken with the $180 \mu \mathrm{m}$ 

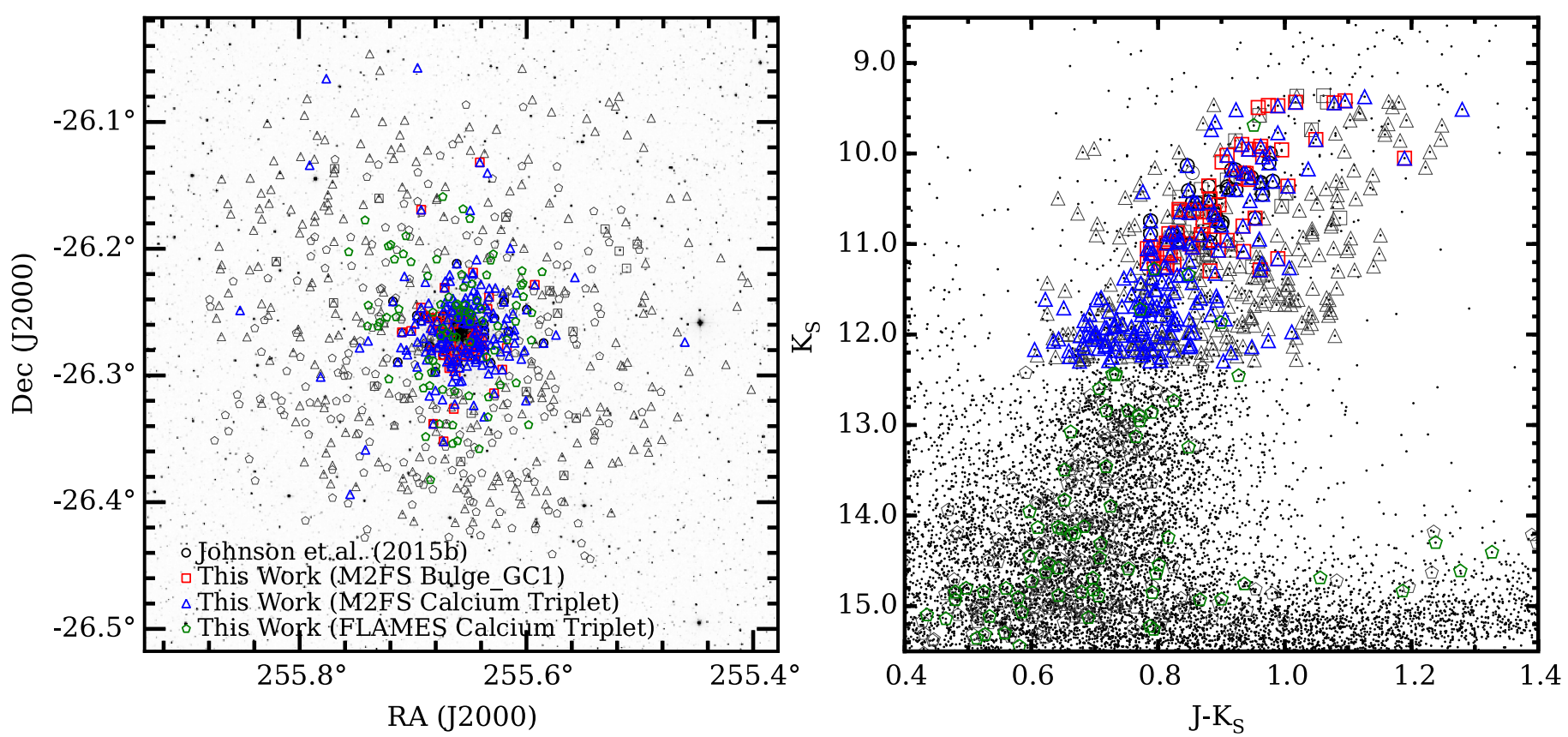

Figure 1. Left: the sky coordinates of all targets observed for this work and Johnson et al. (2015b) are superimposed on a 2MASS (Skrutskie et al. 2006) J-band image centered on NGC 6273. The black, red, blue, and green symbols indicate stars that are radial velocity members, and the gray symbols indicate stars that are likely not cluster members. Right: a 2 MASS $J-K_{\mathrm{S}}$ color-magnitude diagram is shown with the NGC 6273 member and non-member stars indicated using the same symbol and color designations as in the left panel.

Table 1

Observing Log

\begin{tabular}{|c|c|c|c|c|}
\hline Field $^{a}$ & Telescope/Instrument & Setup & UT Date & $\begin{array}{l}\text { Exposure } \\
\text { (s) }\end{array}$ \\
\hline \multicolumn{5}{|c|}{ Spectroscopy } \\
\hline $1 \mathrm{a}$ & VLT-FLAMES & HR21 & 2014 Apr 13 & $1 \times 2445$ \\
\hline $1 b$ & VLT-FLAMES & HR21 & 2014 May 7 & $1 \times 2445$ \\
\hline $2 \mathrm{a}$ & VLT-FLAMES & HR21 & 2014 Jul 13 & $1 \times 2445$ \\
\hline $2 b$ & VLT-FLAMES & HR21 & 2014 Aug 2 & $1 \times 2445$ \\
\hline $3 a$ & VLT-FLAMES & HR21 & 2014 Jul 21 & $1 \times 2445$ \\
\hline $3 b$ & VLT-FLAMES & HR21 & 2014 Aug 13 & $1 \times 2445$ \\
\hline 4 & Magellan-M2FS & $\mathrm{CaT}$ & 2015 Jul 17 & $4 \times 1200$ \\
\hline 5 & Magellan-M2FS & $\mathrm{CaT}$ & 2015 Jul 20 & $3 \times 1800$ \\
\hline 6 & Magellan-M2FS & Bulge_GC1 & $2015 \mathrm{Jul} 21$ & $6 \times 1800$ \\
\hline 7 & Magellan-M2FS & Bulge_GC1 & $2015 \mathrm{Jul} 22$ & $6 \times 1800$ \\
\hline \multicolumn{5}{|c|}{ Photometry } \\
\hline 1 & $H S T-\mathrm{WFC} 3 / \mathrm{UVIS}$ & F336W & 2016 Mar 13 & $4 \times 350$ \\
\hline$\ldots$ & $H S T-\mathrm{WFC} 3 / \mathrm{UVIS}$ & F336W & 2016 Mar 13 & $1 \times 566,659,674,685$ \\
\hline$\ldots$ & $H S T-\mathrm{WFC} 3 / \mathrm{UVIS}$ & $\mathrm{F} 438 \mathrm{~W}$ & 2016 Mar 13 & $2 \times 10$ \\
\hline$\cdots$ & $H S T-$ WFC3/UVIS & F438W & 2016 Mar 13 & $4 \times 350$ \\
\hline$\ldots$ & $H S T-\mathrm{WFC} 3 / \mathrm{UVIS}$ & F555W & 2016 Mar 13 & $4 \times 10$ \\
\hline$\ldots$ & $H S T-\mathrm{WFC} 3 / \mathrm{UVIS}$ & F555W & 2016 Mar 13 & $4 \times 350$ \\
\hline$\cdots$ & $H S T-\mathrm{WFC} 3 / \mathrm{UVIS}$ & F814W & 2016 Mar 13 & $2 \times 10$ \\
\hline$\ldots$ & $H S T-W F C 3 / U V I S$ & F814W & 2016 Mar 13 & $4 \times 350$ \\
\hline
\end{tabular}

Note.

a Fields with different designations indicate different telescope pointings. The "a" and "b" designations for the VLT-FLAMES setups correspond to the "HIERARCH ESO OBS NAME" keyword in the original image headers. The "a" and "b" fields with the same numbers typically observed the same stars, but sometimes with different fibers.

(This table is available in machine-readable form.)

(widest) and $125 \mu \mathrm{m}$ slits, respectively. However, both setups yielded approximately the same resolving power of $R \equiv \lambda / \Delta \lambda$ $\approx 27,000$, based on an examination of the ThAr wavelength calibration spectra. The two CaT fields were observed for a total of 10,200 s, and the two Bulge_GC1 fields were observed for a total of $21,600 \mathrm{~s}$. A summary of the observation dates, instrument configurations, and integration times is provided in Table 1. 
For data reduction, we followed the procedures outlined in Johnson et al. (2015b see their Section 2.3). Briefly, we used standard IRAF ${ }^{13}$ tasks to apply the bias correction, trim the overscan regions, correct for dark current, and combine the individual amplifier images from each CCD into single images. The IRAF dohydra task was used for aperture identification and tracing, flat-field correction, scattered light removal, wavelength calibration, cosmic-ray removal, and spectrum extraction. For the CaT data, we did not apply any corrections for fringing beyond the flat-field correction. A master sky spectrum was created for each exposure by combining the individual sky fiber spectra. The target spectra were then sky corrected using the skysub routine. Finally, the individual extracted spectra for each star were co-added separately, normalized with the continuum routine, and corrected for telluric absorption lines using the telluric task. Typical signalto-noise ratios (S/Ns) ranged from about 20-100 per pixel for the CaT data and 30-100 per pixel for the Bulge_GC1 data.

\subsection{Very Large Telescope (VLT) Spectroscopic Data}

We supplemented the M2FS CaT data set with additional observations of 300 RGB stars taken with the VLT FLAMESGIRAFFE instrument. The data were downloaded from the European Southern Observatory (ESO) Science Archive Facility under request number 210062. ${ }^{14}$ The FLAMES observations spanned a broad range of magnitudes, but were generally fainter than the M2FS data. However, the spatial coverage between the two data sets was similar (see Figure 1). Note that we have only included stars for which we could identify a 2 MASS source within $2^{\prime \prime}$ of the coordinates provided in the image headers.

All of the FLAMES-GIRAFFE observations were obtained using the HR21 setup, which provides $R \approx 18,000$ spectra from 8482 to $9000 \AA$. However, we only analyzed the region spanning $8500-8700 \AA$, which is similar to the M2FS CaT data and includes the same 8542 and $8662 \AA \mathrm{CaT}$ features. The observations were taken via six configurations, each with an integration time of $2445 \mathrm{~s}$. Most stars were observed in two configurations, but not always with the same fiber each time. A small number of stars were observed in three or more configurations, and a few were observed only once. A summary of the observation dates for each configuration is provided in Table 1.

The data were primarily reduced using the GIRAFFE BaseLine Data Reduction Software (girBLDRS ${ }^{15}$ ) package. The girBLDRS suite was used to carry out basic CCD processing tasks (e.g., bias correction and overscan trimming) and also the more advanced multi-fiber tasks we performed with dohydra for the M2FS data (see Section 2.1). Similar to the M2FS CaT data, we did not apply any further corrections for fringing beyond the flat-field correction. The sky subtraction, continuum normalization, and spectrum combining were carried out with the same IRAF routines as used for the M2FS data. However, since the FLAMES data were obtained over the course of several weeks to months, we applied the heliocentric velocity

\footnotetext{
13 IRAF is distributed by the National Optical Astronomy Observatory, which is operated by the Association of Universities for Research in Astronomy, Inc., under cooperative agreement with the National Science Foundation.

${ }^{14}$ Based on observations made with ESO Telescopes at the La Silla Paranal Observatory under program ID 093.D-0628.

${ }^{15}$ The girBLDRS software can be downloaded at http://girbldrs.sourceforge. net/.
}

corrections provided in the image headers before combining the multiple exposures. The final $\mathrm{S} / \mathrm{N}$ values are comparable to those of the M2FS CaT data.

\subsection{HST Imaging Data}

NGC 6273 is known to have a broad RGB and a peculiar HB morphology that is similar to $\omega$ Cen (Piotto et al. 1999; Momany et al. 2004; Brown et al. 2010, 2016; Han et al. 2015). Therefore, in support of our spectroscopic observations, we have obtained new HST Wide Field Camera 3 UVIS channel (WFC3/UVIS) data centered on NGC 6273 that includes the F336W, F438W, F555W, and F814W filters. The observations were split into a series of short and long exposures, taken over the course of four orbits, that ranged in duration from 10 to $685 \mathrm{~s}$. A post-flash of 2.0-4.7 s was included for all exposures, and the $B L A D E=A$ option was set for all of the $10 \mathrm{~s}$ exposures to minimize shutter-induced vibration (see Section 6.11 .4 of the WFC3 handbook ${ }^{16}$ ). A summary of the filter choices, integration times, and observation dates is provided in Table 1.

The basic data reductions were carried out by the Space Telescope Science Institute's WFC3 pipeline, but we only performed analyses on the CTE-corrected $f l c$ images. All photometry was obtained using the DOLPHOT ${ }^{17}$ (Dolphin 2000) package and its associated WFC3 module. The DOLPHOT parameters closely followed the values recommended by Williams et al. (2014) and provided by the DOLPHOT/WFC3 documentation for point sources in crowded fields. No special attempt was made to recover saturated stars; however, only a small number of the brightest stars, predominantly in the $\mathrm{F} 814 \mathrm{~W}$ filter, were lost due to saturation.

As noted by several previous authors (Racine 1973; Harris et al. 1976; Piotto et al. 1999; Davidge 2000; Valenti et al. 2007; Brown et al. 2010; Alonso-García et al. 2012), differential reddening is a significant concern along lines of sight near NGC 6273. Previous work estimated that the cluster has $E(B-V)=0.31-0.47 \mathrm{mag}$ and $\Delta E(B-V) \sim$ $0.2-0.3$ magnitudes. We observe a similar reddening range of $\Delta E(B-V)=0.36$ magnitudes using corrections kindly provided by A. Milone (2016, private communication; see also Milone et al. 2012 for an outline of the dereddening procedure) via the F336W and F814W data sets. Additionally, we find that adopting an absolute color excess of $E(B-V)=0.37 \mathrm{mag}$ places the coolest HB stars at approximately the correct F555W magnitude, assuming a distance of $9 \mathrm{kpc}$ (Piotto et al. 1999). ${ }^{18}$ Further details regarding the photometric analysis, including the dereddening procedure, will be provided in a future publication. However, in Figure 2, we show the smoothed reddening map of the WFC3 field, and include several dereddened color-magnitude diagrams with the radial velocity members identified.

\footnotetext{
16 The WFC3 handbook is available at http://www.stsci.edu/hst/wfc3/ documents/handbooks/currentIHB/.

17 DOLPHOT can be downloaded at http://americano.dolphinsim.com/ dolphot/.

18 Note that we have adopted the extinction coefficients provided by Girardi et al. (2008) and updated at http://stev.oapd.inaf.it/cgi-bin/cmd, for all filters. We have also employed a "standard" extinction curve with $A_{\mathrm{V}}=3.1 E(B-V)$. However, see Udalski (2003), Gosling et al. (2009), and Nataf et al. (2013, 2016) for discussions regarding the validity of adopting a standard extinction curve near the Galactic center.
} 

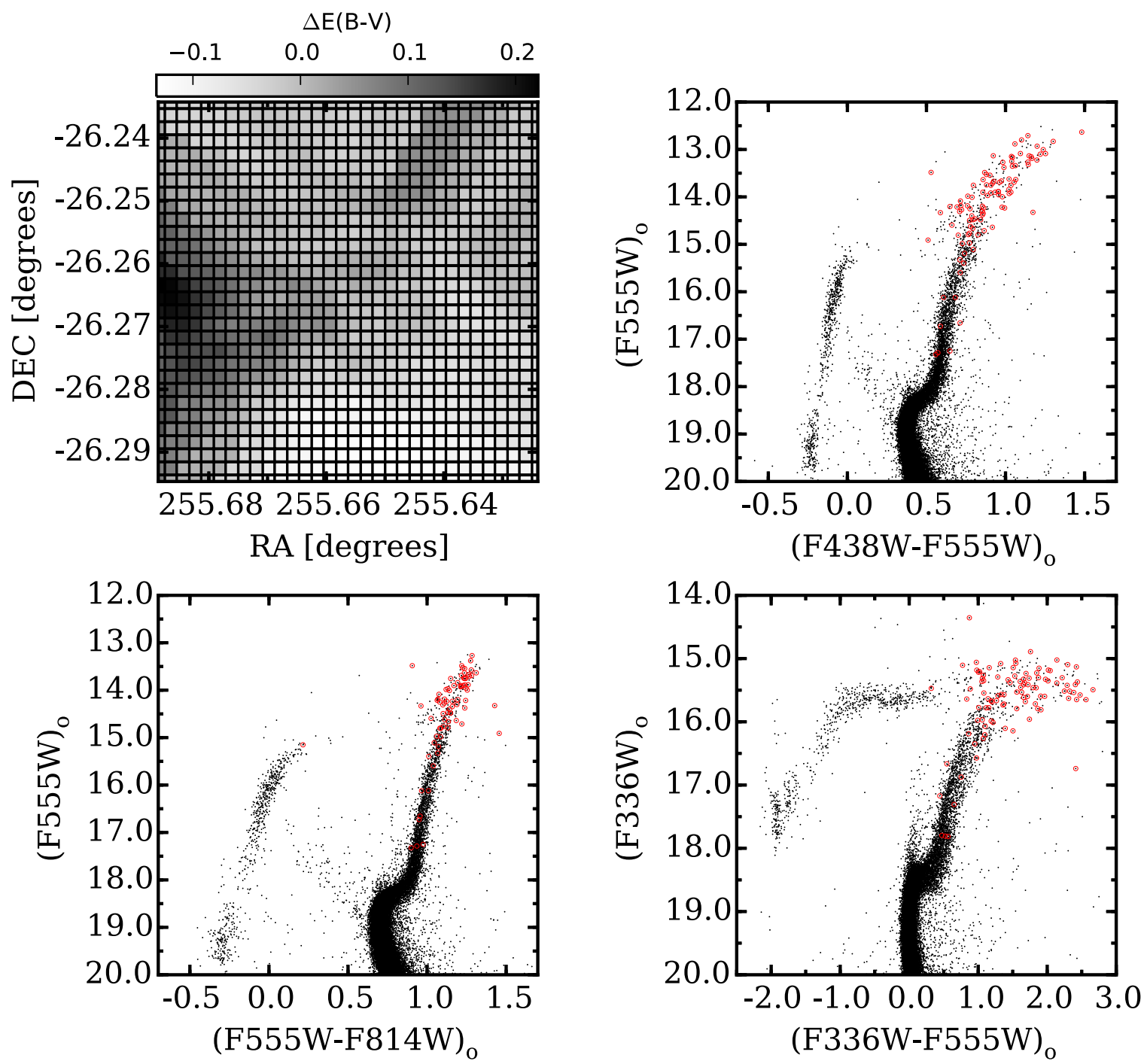

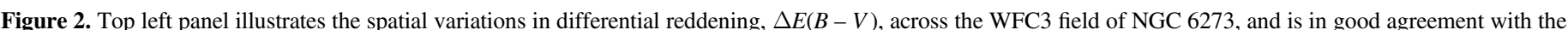

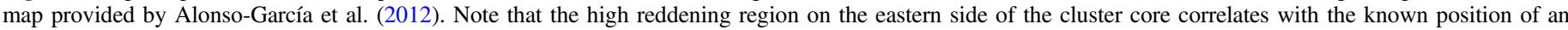

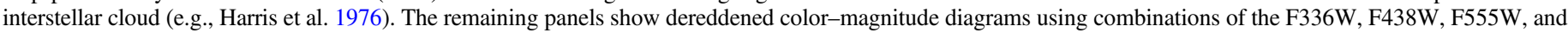

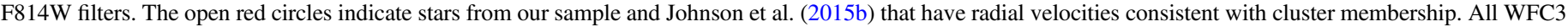
photometry is on the VEGAMAG system.

\section{Radial Velocities and Cluster Membership}

Radial velocities were measured for all M2FS and FLAMES spectra using the XCSAO (Kurtz \& Mink 1998) cross-correlation code. The velocities were measured relative to a synthetic stellar spectrum of an evolved RGB star with $[\mathrm{Fe} / \mathrm{H}]=-1.60$, which is approximately the average metallicity of NGC 6273 (Johnson et al. 2015b). The template spectrum was smoothed and rebinned to match the resolution and sampling of the observed spectra. Heliocentric velocity corrections were calculated with IRAF's rvcorrect utility for the M2FS data, and for the FLAMES data we used the corrections provided in the image headers. The heliocentric corrections were applied to all of the spectra before being measured with XCSAO.

For the Bulge_GC1 spectra, we measured the velocities using the 6140-6270 $\AA$ window because it contains several lines suitable for cross-correlation but avoids very broad lines (e.g., $\mathrm{H} \alpha$ ) and any residual telluric features. For the M2FS and FLAMES CaT data, we used the full spectral window from $8500-8700 \AA$, but avoided the strong CaT lines. A histogram of the heliocentric radial velocity $\left(\mathrm{RV}_{\text {helio. }}\right)$ distributions for each data set, including data from Johnson et al. (2015b), is shown in Figure 3. Using these data, we considered stars with $\mathrm{RV}_{\text {helio. }}$ between +120 and $+170 \mathrm{~km} \mathrm{~s}^{-1}$ to be cluster members. Therefore, the new Bulge_GC1, M2FS CaT, and FLAMES CaT data provided average velocities and dispersions of $+143.15 \mathrm{~km} \mathrm{~s}^{-1}$ $\left(\sigma=9.53 \mathrm{~km} \mathrm{~s}^{-1}\right), \quad+144.74 \mathrm{~km} \mathrm{~s}^{-1} \quad\left(\sigma=8.79 \mathrm{~km} \mathrm{~s}^{-1}\right), \quad$ and $+145.76 \mathrm{~km} \mathrm{~s}^{-1}\left(\sigma=7.12 \mathrm{~km} \mathrm{~s}^{-1}\right)$, respectively, for the cluster members. Similarly, the average $\mathrm{RV}_{\text {helio. }}$ value for the combined data sets is $+144.71 \mathrm{~km} \mathrm{~s}^{-1}\left(\sigma=8.57 \mathrm{~km} \mathrm{~s}^{-1}\right)$, which is in good agreement with recent measurements (Johnson et al. 2015b; Yong et al. 2016). For the non-member stars, we found the average velocity and dispersion to be $-29.36 \mathrm{~km} \mathrm{~s}^{-1}$ and $\sigma=77.02 \mathrm{~km} \mathrm{~s}^{-1}$. These values are in agreement with previous kinematic observations of similar off-axis bulge fields (e.g., Kunder et al. 2012; Ness et al. 2013a; Zoccali et al. 2014).

The average $\mathrm{RV}_{\text {helio. }}$ uncertainties are $0.31 \mathrm{~km} \mathrm{~s}^{-1}(\sigma=$ $\left.0.27 \mathrm{~km} \mathrm{~s}^{-1}\right), 1.09 \mathrm{~km} \mathrm{~s}^{-1}\left(\sigma=0.69 \mathrm{~km} \mathrm{~s}^{-1}\right)$, and $0.88 \mathrm{~km} \mathrm{~s}^{-1}$ $\left(\sigma=0.06 \mathrm{~km} \mathrm{~s}^{-1}\right)$ for the Bulge_GC1, M2FS CaT, and FLAMES $\mathrm{CaT}$ data, respectively. These values represent the measurement 

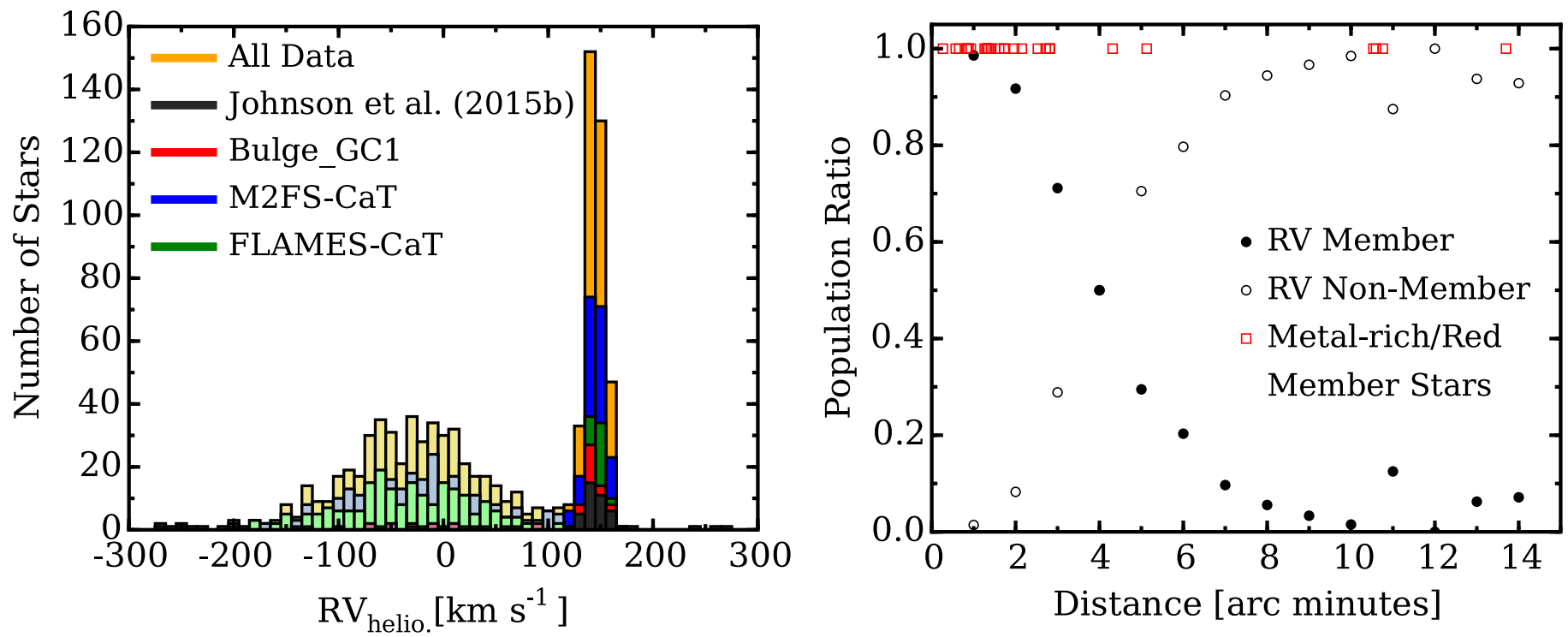

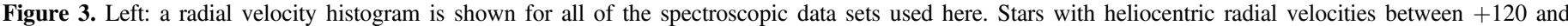

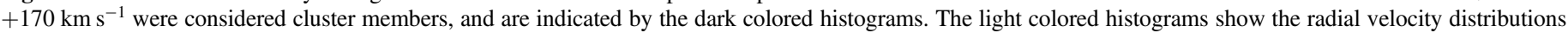

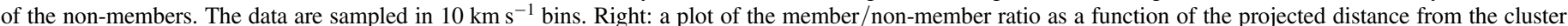

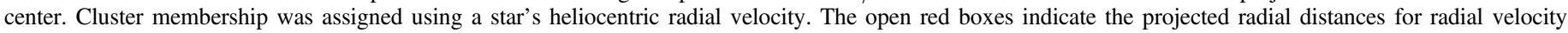
member stars with $[\mathrm{Fe} / \mathrm{H}]>-1.35$ and/or that lie redward of the dominant RGBs seen in Figures 1 and 2.

uncertainties from the XCSAO cross-correlation routine. However, 57 stars were observed in at least two different setups, including the data from Johnson et al. (2015b), and we measured an average dispersion between repeat measurements of $1.31 \mathrm{~km} \mathrm{~s}^{-1}$. If we ignore the four outliers ${ }^{19}$ with dispersions $>5 \mathrm{~km} \mathrm{~s}^{-1}$, the average dispersion decreases to $0.88 \mathrm{~km} \mathrm{~s}^{-1}$. Therefore, we regard $\sim 1 \mathrm{~km} \mathrm{~s}^{-1}$ as a reasonable estimate of the systematic uncertainty due to the use of different instruments, configurations, and wavelength regions.

As can be seen in Figure 3, the systemic cluster velocity is well separated from the broad field star distribution of the Galactic bulge. From the Bulge_GC1, M2FS CaT, and FLAMES CaT data, we found 59/82 (72\%), 191/466 (41\%), and $83 / 300(28 \%)$ stars to have velocities consistent with cluster membership, respectively. The significantly higher membership rate for the Bulge_GC1 data is due to the preferential placement of fibers on stars closer to the cluster core. Both CaT data sets also span a broader color and luminosity range than the Bulge_GC1 observations (see Figure 1).

From the non-member distribution, we estimate that $\sim 0.5 \%$ of field stars will have a velocity between +120 and $+170 \mathrm{~km} \mathrm{~s}^{-1}$ for the lines of sight probed here. Since we have measured velocities for a total of 832 unique stars between the current data sets and Johnson et al. (2015b), we expect $\sim 5$ field stars in the combined data to have velocities consistent with cluster membership. However, the field star contamination rate may be overestimated because the cluster and field stars do not share the same spatial and metallicity distributions.

Figures 1 and 3 show that a majority of stars having velocities consistent with cluster membership reside inside $4^{\prime}$ of the cluster center, but the obvious field stars are more uniformly distributed. Additionally, Johnson et al. (2015b) and Yong et al. (2016) have shown that most NGC 6273 stars have $[\mathrm{Fe} / \mathrm{H}] \lesssim-1.35$, but such stars are relatively rare in the bulge field (e.g., Zoccali et al.

\footnotetext{
19 Note that we have not rejected the outlier stars from the list of member stars nor the chemical abundance analysis.
}

2008; Bensby et al. 2013; Johnson et al. 2013; Ness et al. 2013b). The most likely contaminators are therefore stars that lie $\gtrsim 4^{\prime}$ from the cluster center and have very red colors and/ or $[\mathrm{Fe} / \mathrm{H}]>-1.35$. Figure 3 indicates that six such stars exist in our data set. Of these, stars 2MASS 17030978-2608035 and 17030625-2603576 are the most likely to be field stars because both have $[\mathrm{Fe} / \mathrm{H}]>-0.8$ and radial distances of $>10^{\prime}$. Star 2MASS 17024153-2621081 has $[\mathrm{Fe} / \mathrm{H}]=-1.53$, a radial distance of $5 ! 1$, and is likely a cluster member. The three remaining candidates (2MASS 17015056-2616256; 2MASS 17032450-2614557; 2MASS 17023960-2620224) have distances of $4 ! 3-10 ! 6$ but lack $[\mathrm{Fe} / \mathrm{H}]$ measurements so their membership cannot yet be confirmed. Listings of star identifications, coordinates, photometry, and heliocentric radial velocities for member and non-member stars are provided in Tables 2 and 3 , respectively.

Establishing membership near and beyond the tidal radius (14'.57; Alonso-García et al. 2012) will be important in searches for any extended halo populations associated with NGC 6273, similar to what is observed near clusters such as NGC 1851, M2, NGC 5824, M3, and M13 (Grillmair et al. 1995; Olszewski et al. 2009; Marino et al. 2014b; Navin et al. 2015, 2016; Kuzma et al. 2016). Figure 1 shows a possibly interesting morphology such that stars near the edge of our observations, which are also close to the tidal radius, are more numerous on the eastern side of the cluster than the western side. However, more observations are needed to confirm that this asymmetry is real.

\subsection{Cluster Rotation}

Many globular clusters have been shown to rotate with amplitudes of the order of a few $\mathrm{km} \mathrm{s}^{-1}$ (e.g., Côté et al. 1995; Lane et al. 2009, 2010a; Bellazzini et al. 2012; Bianchini et al. 2013; Kacharov et al. 2014; Kimmig et al. 2015; Lardo et al. 2015). In Figure 4, we investigated net rotation in NGC 6273 by following a standard technique in which the average radial velocity is calculated for stars on either side of an imaginary line 
Table 2

Star Identifiers, Coordinates, Photometry, and Radial Velocities for NGC 6273 Members

\begin{tabular}{|c|c|c|c|c|c|c|}
\hline $\begin{array}{l}\text { Star Name } \\
\text { (2MASS) }\end{array}$ & $\begin{array}{c}\text { R.A. } \\
\text { (degrees) }\end{array}$ & $\begin{array}{c}\text { Decl. } \\
\text { (degrees) }\end{array}$ & $\begin{array}{c}J \\
\text { (mag.) }\end{array}$ & $\begin{array}{c}K_{\mathrm{S}} \\
\text { (mag.) }\end{array}$ & $\begin{array}{l}\mathrm{RV}_{\text {helio. }} \\
\left(\mathrm{km} \mathrm{s}^{-1}\right)\end{array}$ & $\begin{array}{l}\text { RV Error } \\
\left(\mathrm{km} \mathrm{s}^{-1}\right)\end{array}$ \\
\hline \multicolumn{7}{|c|}{ Bulge_GC1 Members } \\
\hline $17022817-2616426$ & 255.617398 & -26.278500 & 11.893 & 11.102 & 156.50 & 0.23 \\
\hline $17022912-2617443^{\mathrm{d}}$ & 255.621349 & -26.295652 & 11.153 & 10.218 & 143.61 & 0.18 \\
\hline 17023087-2618515 & 255.628646 & -26.314312 & 11.891 & 11.103 & 149.94 & 0.27 \\
\hline $17023338-2617104^{\mathrm{d}}$ & 255.639093 & -26.286234 & 12.075 & 11.262 & 143.75 & 0.37 \\
\hline
\end{tabular}

Notes.

a Observed in Johnson et al. (2015b), the Bulge_GC1 setup, and the M2FS Calcium Triplet setup.

b Observed in Johnson et al. (2015b) and the Bulge_GC1 setup.

${ }^{c}$ Observed in Johnson et al. (2015b) and the M2FS Calcium Triplet setup.

d Observed in the Bulge_GC1 and M2FS Calcium Triplet setups.

e Observed in the M2FS Calcium Triplet and FLAMES Calcium Triplet setups.

(This table is available in its entirety in machine-readable form.)

Table 3

Star Identifiers, Coordinates, Photometry, and Radial Velocities for Non-members

\begin{tabular}{|c|c|c|c|c|c|c|}
\hline $\begin{array}{l}\text { Star Name } \\
\text { (2MASS) }\end{array}$ & $\begin{array}{c}\text { R.A. } \\
\text { (degrees) }\end{array}$ & $\begin{array}{c}\text { Decl. } \\
\text { (degrees) }\end{array}$ & $\begin{array}{c}J \\
\text { (mag.) }\end{array}$ & $\begin{array}{c}K_{\mathrm{S}} \\
\text { (mag.) }\end{array}$ & $\begin{array}{l}\mathrm{RV}_{\text {helio. }} \\
\left(\mathrm{km} \mathrm{s}^{-1}\right)\end{array}$ & $\begin{array}{c}\text { RV Error } \\
\left(\mathrm{km} \mathrm{s}^{-1}\right)\end{array}$ \\
\hline \multicolumn{7}{|c|}{ Bulge_GC1 Non-Members } \\
\hline $17020290-2612561$ & 255.512110 & -26.215591 & 11.410 & 10.543 & -69.71 & 0.17 \\
\hline $17020445-2612074$ & 255.518548 & -26.202074 & 10.501 & 9.434 & 5.64 & 0.32 \\
\hline $17020743-2611048$ & 255.530968 & -26.184685 & 10.423 & 9.362 & 94.73 & 0.32 \\
\hline $17021609-2622447$ & 255.567065 & -26.379099 & 11.789 & 10.937 & 65.75 & 0.34 \\
\hline 17021744-2615041 & 255.572691 & -26.251162 & 12.029 & 11.174 & 42.82 & 0.33 \\
\hline
\end{tabular}

Notes.

${ }^{a}$ Observed in Johnson et al. (2015b) and the M2FS Calcium Triplet setup.

${ }^{\mathrm{b}}$ Observed in the Bulge_GC1 and M2FS Calcium Triplet setups.

(This table is available in its entirety in machine-readable form.)

passing through the cluster center. The bisecting line is rotated east through west in $10^{\circ}$ increments, and the velocity differences are plotted as a function of position angle. The resulting data can be fit with a sinusoidal function of the form

$$
\Delta\left\langle V_{r}\right\rangle=A_{\text {rot. }} \sin (\mathrm{PA}+\Phi),
$$

where $A_{\text {rot. }}$ is twice the actual projected rotation amplitude, $\Phi=270^{\circ}-\mathrm{PA}_{\circ}$, and $\mathrm{PA}_{\circ}$ is the angle of maximum rotation. Bellazzini et al. (2012) argue that the projected $A_{\text {rot. }}$ value should be a reasonable estimate for the true maximum rotation amplitude, and we have adopted their interpretation here.

For NGC 6273 , we find a clear rotation signature with $A_{\text {rot. }}=3.83 \pm 0.12 \mathrm{~km} \mathrm{~s}^{-1}$ and $\mathrm{PA}_{\mathrm{o}}=126^{\circ} \pm 2^{\circ}$. We calculated the rotation profile using various angular bin sizes and found that, while $A_{\text {rot. }}$ only varied by a few tenths of a $\mathrm{km} \mathrm{s}^{-1}$, the PAo value could change by $\sim 15^{\circ}$. Therefore, we follow Bellazzini et al. (2012) and have adopted the conservative $1 \sigma$ uncertainties of $\pm 0.5 \mathrm{~km} \mathrm{~s}^{-1}$ for $A_{\text {rot }}$ and $\pm 30^{\circ}$ for $\mathrm{PA}_{\text {o. }}$. Compared to the large globular cluster samples presented in Bellazzini et al. (2012), Kimmig et al. (2015), and Lardo et al. (2015), NGC 6273 exhibits relatively strong rotation. NGC 6273's large $A_{\text {rot. }}$ value is consistent with other clusters having similar metallicity and mass (e.g., $\omega$ Cen; see Figures 11 and 19 in Bellazzini et al. 2012 and Lardo et al. 2015, respectively).

In Figure 5, we also investigated the change in velocity dispersion as a function of the projected radial distance from the cluster center. As expected, we find that the velocity dispersion decreases from at least $10 \mathrm{~km} \mathrm{~s}^{-1}$ inside $1^{\prime}$ to less than $5 \mathrm{~km} \mathrm{~s}^{-1}$ outside $5^{\prime}$. We also estimated the cluster's central velocity dispersion $\left(\sigma_{\mathrm{o}}\right)$ using simple Plummer models (Plummer 1911) of the form

$$
\sigma^{2}(r)=\frac{\sigma_{o}^{2}}{\sqrt{1+\left(\frac{r}{r_{h}}\right)^{2}}},
$$

where $r_{\mathrm{h}}$ is the Plummer scale radius. ${ }^{20}$ We fit two models: (1) one with both $\sigma_{\mathrm{o}}$ and $r_{\mathrm{h}}$ varied as free parameters and (2) one with $\sigma_{\mathrm{o}}$ varied as a free parameter and $r_{\mathrm{h}}$ held fixed. For the

\footnotetext{
${ }^{20}$ As noted in Lane et al. (2010b), the Plummer scale radius is equivalent to the projected half-mass-radius for projected Plummer models.
} 

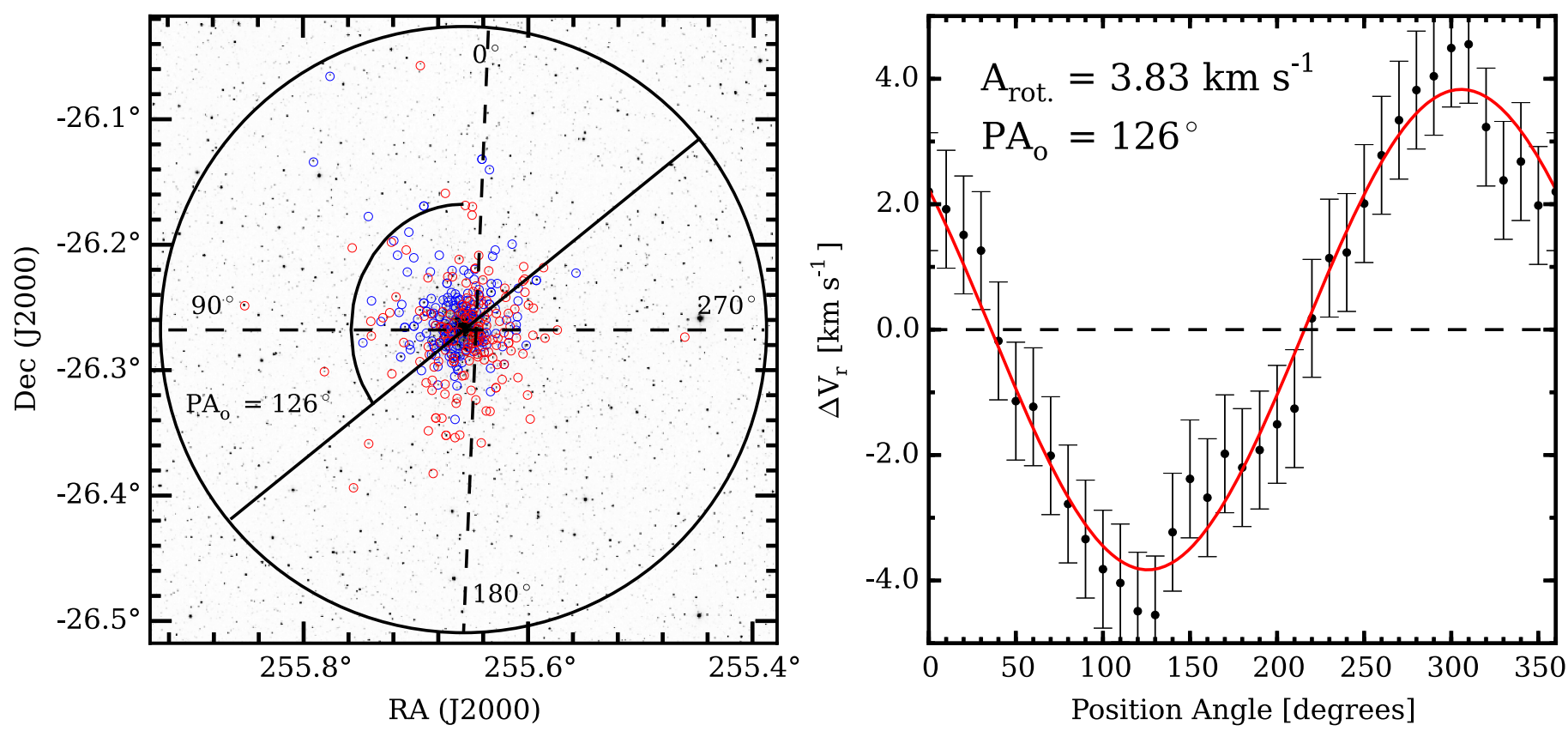

Figure 4. Left: the sky coordinates of member stars with heliocentric radial velocities lower (blue) and higher (red) than the cluster average are superimposed on a 2MASS J-band image. The solid black line bisecting the cluster illustrates the position angle of the rotation axis (PAo), which is measured by rotating the solid black line east through west and finding the maximum difference in heliocentric radial velocity on each side. Right: the average heliocentric radial velocity difference for position angles measured in $10^{\circ}$ increments. The solid red line indicates the best-fit sinusoidal function to the data. See the text for details.
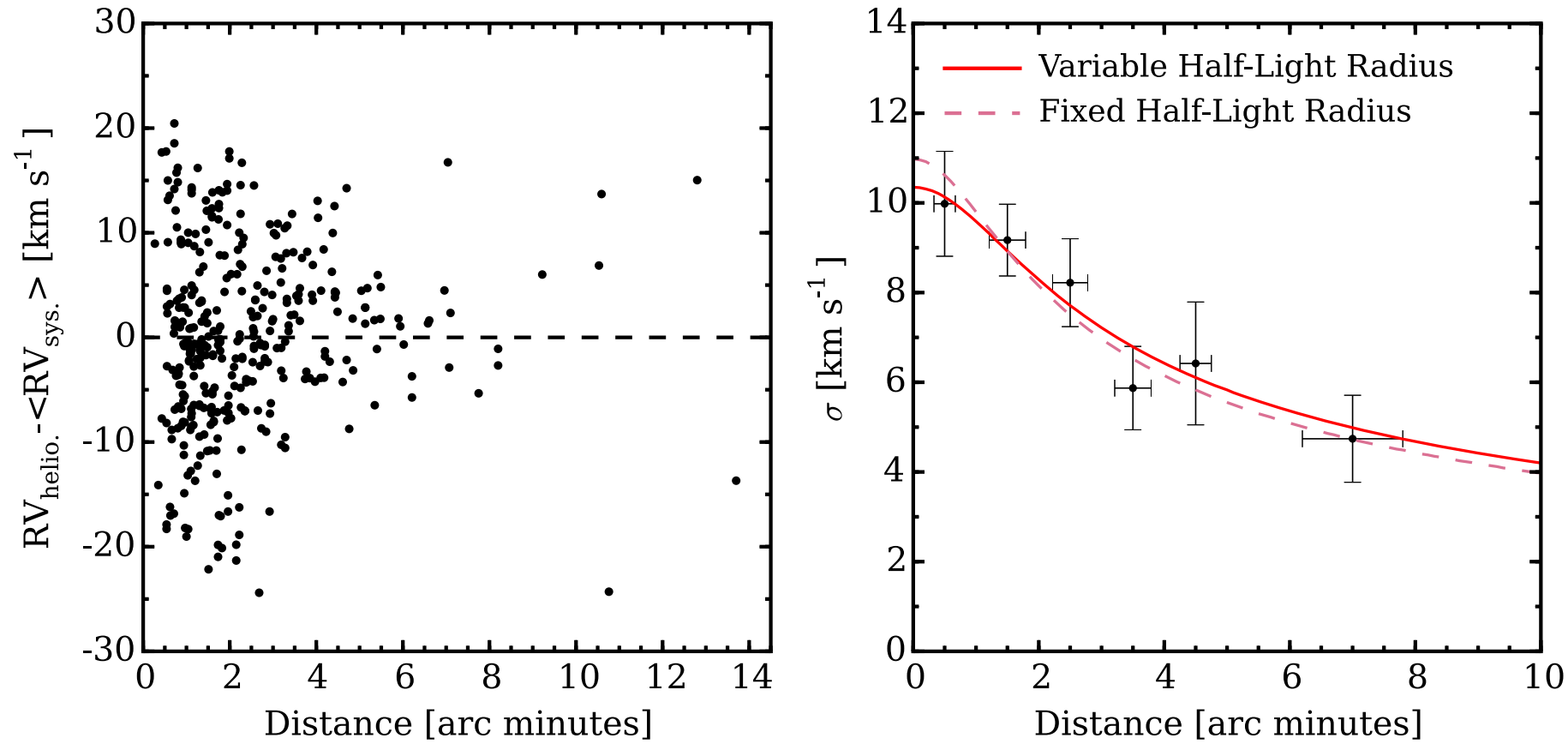

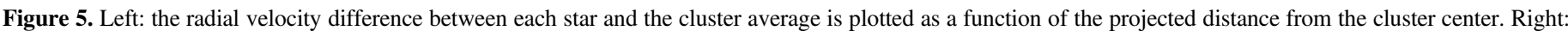

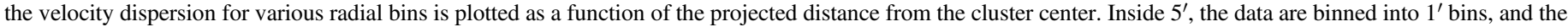

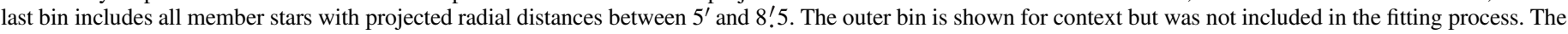

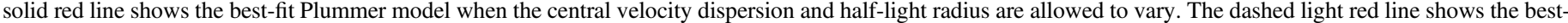
fit Plummer model when the half-light radius is held fixed. See the text for details.

latter case, we assumed the half-light radius was approximately equal to the half-mass radius and adopted a half-light radius of 1!32 (Harris 1996; 2010 revision). The resulting fit provided $\sigma_{\mathrm{o}}=10.98 \pm 0.40 \mathrm{~km} \mathrm{~s}^{-1}$. For the former case, we found $\sigma_{\mathrm{o}}=10.35 \pm 0.69 \mathrm{~km} \mathrm{~s}^{-1}$ and $\mathrm{r}_{\mathrm{h}}=1 ! 67 \pm 0 ! 41$.
However, we regard these values as lower limits of the true central velocity dispersion because the measured velocity dispersion for the bin closest to the cluster core is sensitive to the adopted bin size. For example, when the first bin contains stars with projected radial distances of $0 ! 2-1 ! 0$, as is done in Figure 5 , the dispersion is $\sim 10 \mathrm{~km} \mathrm{~s}^{-1}$, but if we change the 
range to $0 ! 2-0 ! 7$, then the dispersion increases to $\sim 12 \mathrm{~km} \mathrm{~s}^{-1}$. Furthermore, a simple Plummer model assumes spherical symmetry, but NGC 6273 is relatively elliptical in shape (White \& Shawl 1987; Chen \& Chen 2010). Additional velocity measurements inside $\sim 0$ !.2-0!.5 and the application of more sophisticated models are likely to find a true $\sigma_{\mathrm{o}}>12$ $\mathrm{km} \mathrm{s}^{-1}$. We estimate that the cluster's true $A_{\text {rot. }} / \sigma_{\mathrm{o}}$ ratio is $\sim 0.30-0.35$, which is typical for massive elliptical metal-poor globular clusters (e.g., see Bellazzini et al. 2012; Kacharov et al. 2014; Kimmig et al. 2015; Lardo et al. 2015).

\section{Spectroscopic Analysis}

\subsection{Model Atmospheres}

The model atmosphere parameters effective temperature $\left(T_{\text {eff }}\right)$, surface gravity $(\log (g))$, metallicity $([\mathrm{Fe} / \mathrm{H}])$, and microturbulence $\left(\xi_{\text {mic }}\right)$ were determined spectroscopically for all radial velocity member stars observed with the Bulge_GC1 setup. A spectroscopic determination of especially $T_{\text {eff }}$ and $\log (g)$ is preferred over photometric measurements for NGC 6273 because of the cluster's large and variable reddening (see Section 2.3). We followed the general analysis procedures outlined in Johnson et al. (2015b), which includes the use of the 1D local thermodynamic equilibrium (LTE) line analysis code MOOG $^{21}$ (Sneden 1973; 2014 version). In particular, we solved for $T_{\text {eff }}$ by enforcing excitation equilibrium with the Fe I lines and solved for surface gravity by adjusting $\log (g)$ until the $\mathrm{Fe} \mathrm{I}$ and $\mathrm{Fe}$ II lines provided the same abundance. In the few instances where only Fe I could be measured, we assigned stars a $\log (g)$ value that was compatible with other cluster members of similar temperature and metallicity. Microturbulence was measured by adjusting $\xi_{\text {mic. }}$ until the derived $\log \epsilon(\mathrm{Fe} \mathrm{I})$ abundance was independent of line strength. Finally, the metallicity of each model was set as the average of $[\mathrm{Fe} \mathrm{I} / \mathrm{H}]$ and $[\mathrm{Fe} \mathrm{II} / \mathrm{H}]$.

In order to generate the models, we interpolated within the available grid of ATLAS9 model atmospheres ${ }^{22}$ (Castelli \& Kurucz 2004). For most stars, we used the $\alpha$-enhanced models in order to compensate for the difference between $[\mathrm{Fe} / \mathrm{H}]$ and $[\mathrm{M} / \mathrm{H}]$. However, a small number of stars in our sample have $[\alpha / \mathrm{Fe}] \sim 0$, and for those stars we used the scaled-solar models. For every star, we started with a base-line model of $T_{\text {eff }}=4500 \mathrm{~K}, \log (g)=1.20 \mathrm{cgs},[\mathrm{Fe} / \mathrm{H}]=-1.60 \mathrm{dex}$, and $\xi_{\text {mic. }}=1.70 \mathrm{~km} \mathrm{~s}^{-1}$, and iteratively solved for all four parameters simultaneously.

Lind et al. (2012) showed that, for some stars, departures from LTE can have a significant impact on the model atmosphere parameters derived by spectroscopic methods. However, the impact on stars in the temperature, gravity, and metallicity regime probed here is likely to be small. Additionally, the relative effects due to departures from LTE should be mostly negligible within a small parameter space (e.g., Wang et al. 2016), and we have attempted to empirically cancel out large non-LTE and 3D model atmosphere deficiencies by performing a differential analysis relative to Arcturus. Therefore, we have not applied any non-LTE corrections to our data. We note also that Dupree et al. (2016) showed the addition of a chromosphere may alter the

\footnotetext{
21 The MOOG source code is available at http://www.as.utexas.edu/ chris/ moog.html.

${ }^{22}$ The model atmosphere grid can be accessed at http://wwwuser.oats.inaf.it/ castelli/grids.html.
}

derived abundances for some elements. However, since we lack the spectral coverage necessary for constraining a chromospheric model, our model atmosphere parameters and abundances are based only on radiative/convective equilibrium models. The final model atmosphere parameters for all member stars derived from the Bulge_GC1 data are provided in Table 4.

\subsection{Equivalent Width (EW) and Spectrum Synthesis Measurements}

The abundances of Si I, Ca I, Cr I, Fe I, Fe II, and Ni I were obtained by measuring the EW of individual lines selected by Johnson et al. (2015b) to be relatively free of contamination from significant blends and residual telluric features. On average, the Si I, Ca I, Cr I, Fe I, Fe II, and Ni I abundances were based on the measurement of 2, 5, 2, 33, 4, and 4 absorption lines, respectively. However, we only measured the abundances of these elements from the Bulge_GC1 spectra. We utilized the same EW measuring code, line list, and solar reference abundances described in Johnson et al. (2015b see their Section 3.2 and their Table 2), and also used the same abfind driver in MOOG to calculate the final abundance ratios. The $[\mathrm{Si} \mathrm{I} / \mathrm{Fe}],[\mathrm{Ca} \mathrm{I} / \mathrm{Fe}],[\mathrm{Cr} \mathrm{I} / \mathrm{Fe}],[\mathrm{Fe} \mathrm{I} / \mathrm{H}],[\mathrm{Fe} \mathrm{II} / \mathrm{H}]$, and $[\mathrm{Ni} \mathrm{I} / \mathrm{Fe}]$ abundances for every cluster member observed in the Bulge_GC1 setup are provided in Tables 5-6.

The abundances of $\mathrm{NaI}, \mathrm{Mg}$ I, Al I, La II, and Eu II were obtained by using the synth driver in MOOG to fit synthetic spectra to the observations. The synthetic spectra were calculated using the line list developed for Johnson et al. (2015b), which is tuned to reproduce the Arcturus spectrum near the lines of interest and includes the updated $\mathrm{CN}$ line list from Sneden et al. (2014). We preferred to use spectrum synthesis rather than an EW analysis for these elements because their abundances are more sensitive to blending, contamination from other features, and/or broadening effects. For example, the $\mathrm{Na}$ and $\mathrm{Al}$ lines can have significant contamination from nearby atomic features and molecular $\mathrm{CN}$, especially in the more metal-rich stars. Additionally, the $\mathrm{Mg}$ triplet near $6319 \AA$ contains very weak lines, and the nearby continuum can be affected by a shallow but broad $\mathrm{Ca}$ I autoionization feature. The $\mathrm{La}$ and $\mathrm{Eu}$ lines are also relatively weak, but are further affected by hyperfine structure broadening. The Eu lines also contain a mixture of transitions from the ${ }^{151} \mathrm{Eu}$ and ${ }^{153} \mathrm{Eu}$ isotopes, for which we assumed the ${ }^{151} \mathrm{Eu}:{ }^{153} \mathrm{Eu}$ Solar System ratio of $47.8 \%: 52.2 \%$ (Lawler et al. 2001).

The final $[\mathrm{Na} / \mathrm{Fe}],[\mathrm{Mg} / \mathrm{Fe}],[\mathrm{Al} / \mathrm{Fe}],[\mathrm{La} / \mathrm{Fe}]$, and $[\mathrm{Eu} / \mathrm{Fe}]$ abundances derived for cluster members observed with the Bulge_GC1 setup are provided in Tables 5-6. All atomic parameters and solar reference abundances are available in Johnson et al. (2015b; their Table 2).

\subsection{Calcium Triplet Abundances}

In addition to the $[\mathrm{Fe} / \mathrm{H}]$ abundances derived from the EW measurements of individual Fe I and Fe II lines, we measured $[\mathrm{Fe} / \mathrm{H}]$ in a larger sample of stars using the 8542 and $8662 \AA$ $\mathrm{CaT}$ lines. These strong lines have been shown to be sensitive to a star's metallicity and relatively insensitive to a star's age or $[\alpha / \mathrm{Fe}]$ abundance, in a variety of environments (e.g., Armandroff \& Da Costa 1991; Olszewski et al. 1991; Idiart et al. 1997; Rutledge et al. 1997; Cole et al. 2004; Carrera et al. 2007; Battaglia et al. 2008; Da Costa 2016b). Although several CaT metallicity calibrations exist (e.g., Starkenburg 
Table 4

Model Atmosphere Parameters for NGC 6273 Members

\begin{tabular}{|c|c|c|c|c|}
\hline $\begin{array}{l}\text { Star Name } \\
\text { (2MASS) }\end{array}$ & $\begin{array}{l}T_{\text {eff }} \\
(\mathrm{K})\end{array}$ & $\begin{array}{c}\log (g) \\
(\operatorname{cgs})\end{array}$ & $\begin{array}{c}{[\mathrm{Fe} / \mathrm{H}]} \\
(\mathrm{dex})\end{array}$ & $\underset{\left(\mathrm{km} \mathrm{s}^{-1}\right)}{\xi_{\text {mic. }}}$ \\
\hline $17022227-2613433^{\mathrm{c}}$ & 4400 & 1.15 & -1.67 & 1.90 \\
\hline $17022817-2616426$ & 4675 & 1.75 & -1.66 & 1.95 \\
\hline $17022912-2617443^{\mathrm{c}}$ & 4325 & 0.80 & -1.80 & 1.80 \\
\hline $17023087-2618515$ & 4575 & 1.10 & -1.86 & 1.95 \\
\hline $17023192-2614177^{\mathrm{c}}$ & 4325 & 1.35 & -1.49 & 2.10 \\
\hline $17023225-2614521$ & 4500 & 1.15 & -1.71 & 1.85 \\
\hline $17023338-2617104^{\mathrm{c}}$ & 4675 & 1.50 & -1.63 & 1.85 \\
\hline $17023342-2616165$ & 4575 & 1.65 & -1.51 & 1.75 \\
\hline $17023346-2616375$ & 4700 & 1.95 & -1.42 & 1.80 \\
\hline $17023388-2607556$ & 4600 & 1.15 & -1.78 & 2.05 \\
\hline 17023394-2616196 & 4250 & 0.20 & -1.94 & 2.05 \\
\hline $17023435-2616386$ & 4200 & 0.70 & -1.77 & 1.90 \\
\hline $17023459-2615560^{\mathrm{c}}$ & 4250 & 0.85 & -1.85 & 1.90 \\
\hline $17023460-2616038$ & 4625 & 1.25 & -1.72 & 1.90 \\
\hline $17023517-2616130$ & 4400 & 1.30 & -1.39 & 1.65 \\
\hline $17023523-2617058$ & 4350 & 1.20 & -1.52 & 1.70 \\
\hline $17023529-2613089^{c}$ & 4500 & 1.40 & -1.63 & 1.95 \\
\hline $17023551-2616175$ & $\cdots$ & $\cdots$ & $\cdots$ & $\cdots$ \\
\hline $17023583-2616444$ & 4775 & 1.70 & -1.70 & 1.80 \\
\hline $17023589-2615218$ & 4775 & 1.90 & -1.56 & 1.85 \\
\hline $17023595-2615342^{\mathrm{c}}$ & 4350 & 1.55 & -1.22 & 2.30 \\
\hline $17023618-2616576$ & 4800 & 1.90 & -1.55 & 1.70 \\
\hline $17023685-2616454^{\mathrm{c}}$ & 4650 & 1.60 & -1.78 & 1.90 \\
\hline $17023694-2615130$ & 4900 & 2.15 & -1.48 & 1.70 \\
\hline $17023720-2614581^{\mathrm{a}}$ & 4900 & 2.15 & -1.54 & 1.70 \\
\hline $17023723-2617063$ & 4400 & 1.25 & -1.64 & 1.80 \\
\hline $17023728-2617024$ & 4500 & 1.05 & -1.83 & 1.75 \\
\hline $17023744-2615306$ & 4650 & 1.55 & -1.78 & 1.35 \\
\hline $17023783-2615095^{\mathrm{c}}$ & $\ldots$ & $\ldots$ & $\ldots$ & $\ldots$ \\
\hline $17023898-2618010$ & 4650 & 1.65 & -1.48 & 1.50 \\
\hline $17023916-2616500$ & 4300 & 1.00 & -1.71 & 1.70 \\
\hline $17023938-2619361$ & 4550 & 1.15 & -1.71 & 1.90 \\
\hline $17023943-2615343$ & 4575 & 1.10 & -1.70 & 1.95 \\
\hline $17023946-2615017^{\mathrm{a}}$ & 4800 & 2.00 & -1.49 & 1.50 \\
\hline $17023956-2617202^{\mathrm{c}}$ & 4850 & 2.10 & -1.45 & 1.80 \\
\hline $17023984-2617360^{\mathrm{a}}$ & 4500 & 1.50 & -1.41 & 1.80 \\
\hline $17023993-2616370^{\mathrm{c}}$ & $\cdots$ & $\cdots$ & $\cdots$ & $\cdots$ \\
\hline $17024016-2615588$ & $\cdots$ & $\cdots$ & $\cdots$ & $\cdots$ \\
\hline $17024032-2617400$ & 4700 & 1.95 & -1.44 & 1.80 \\
\hline $17024041-2617149$ & 4550 & 1.25 & -1.70 & 1.80 \\
\hline $17024104-2616507^{\mathrm{b}}$ & 4600 & 1.25 & -1.74 & 1.65 \\
\hline $17024128-2616015$ & $\cdots$ & $\cdots$ & $\cdots$ & $\cdots$ \\
\hline $17024132-2613517^{\mathrm{a}}$ & $\cdots$ & $\cdots$ & $\cdots$ & $\cdots$ \\
\hline $17024153-2621081$ & 4250 & 0.85 & -1.53 & 2.10 \\
\hline $17024165-2617033^{b}$ & 4550 & 1.25 & -1.90 & 1.75 \\
\hline $17024173-2616245$ & 4225 & 1.10 & -1.57 & 1.95 \\
\hline $17024226-2615137$ & 4500 & 1.40 & -1.59 & 2.10 \\
\hline $17024242-2615557$ & 4425 & 1.40 & -1.60 & 1.85 \\
\hline $17024289-2615274^{\mathrm{a}}$ & 4650 & 1.10 & -1.70 & 1.85 \\
\hline $17024371-2620183^{\mathrm{a}}$ & 4500 & 1.20 & -1.73 & 1.70 \\
\hline $17024377-2615526^{\mathrm{c}}$ & 4475 & 1.50 & -1.42 & 1.90 \\
\hline $17024412-2616495$ & 4475 & 1.20 & -1.51 & 1.90 \\
\hline $17024416-2615177^{b}$ & 4800 & 1.95 & -1.44 & 2.00 \\
\hline $17024472-2615190$ & $\cdots$ & $\cdots$ & $\cdots$ & $\cdots$ \\
\hline $17024566-2615124^{a}$ & 4775 & 2.40 & -1.09 & 2.00 \\
\hline $17024625-2610100$ & 4400 & 0.75 & -2.00 & 1.90 \\
\hline $17024627-2614484^{\mathrm{c}}$ & $\ldots$ & $\cdots$ & $\ldots$ & $\cdots$ \\
\hline $17024838-2615546$ & 4250 & 0.70 & -1.54 & 2.00 \\
\hline $17025033-2615582^{\mathrm{a}}$ & 4575 & 2.00 & -1.27 & 1.80 \\
\hline
\end{tabular}

Notes.

${ }^{a}$ Observed in Johnson et al. (2015b), the Bulge_GC1 setup, and the M2FS Calcium Triplet setup.

${ }^{\mathrm{b}}$ Observed in Johnson et al. (2015b) and the Bulge_GC1 setup.

${ }^{c}$ Observed in the Bulge_GC1 and M2FS Calcium Triplet setups.

(This table is available in machine-readable form.)

et al. 2010; Saviane et al. 2012; Carrera et al. 2013; Vásquez et al. 2015), we followed the technique outlined in Yong et al. (2016) that utilizes the Mauro et al. (2014) calibration.
As noted by Yong et al. (2016), the Mauro et al. (2014) calibration has two significant advantages for NGC 6273: (1) the luminosity component of the calibration depends on a star's $K_{\mathrm{S}}$ magnitude, rather than $V$ magnitude, which is much less affected by differential reddening; and (2) the significantly flatter slope of the summed EW ( $\Sigma \mathrm{EW})$ versus $\mathrm{K}_{\mathrm{S}}(\mathrm{HB})-\mathrm{K}_{\mathrm{S}}$ relation reduces the effects of photometric, distance, and reddening uncertainties on the derived $[\mathrm{Fe} / \mathrm{H}]$ values. Additionally, we note that $2 \mathrm{MASS}$ provides uniform $K_{\mathrm{S}}$ photometry for our entire sample, but uniform $V$ magnitudes are not yet available for all stars. However, since most CaT-metallicity relations may only be reliable down to the luminosity level of the HB (e.g., Da Costa et al. 2009), we did not determine CaT metallicities for stars fainter than the HB. This cut-off primarily affected the FLAMES CaT sample.

The Mauro et al. (2014) calibration requires a measurement of the summed $8542 \AA$ and $8662 \AA$ CaT EWs, defined as

$$
\Sigma \mathrm{EW}=\mathrm{EW}_{8542}+\mathrm{EW}_{8662},
$$

and the value $K_{\mathrm{S}}(\mathrm{HB})-\mathrm{K}_{\mathrm{S}}$, where $K_{\mathrm{S}}(\mathrm{HB})$ is the magnitude of the HB. Following Yong et al. (2016), we have adopted $K_{\mathrm{S}}(\mathrm{HB})=12.85 \mathrm{mag}$ (Valenti et al. 2007). The EWs for each line were fit using a function that is the sum, rather than the convolution, of a Gaussian and Lorentzian profile. Using Equation (7) and following Mauro et al. (2014), we adopted their relation,

$$
\Sigma \mathrm{EW}=-0.385\left[K_{S}(\mathrm{HB})-K_{S}\right]+W^{\prime},
$$

to solve for the reduced $\mathrm{EW}\left(W^{\prime}\right)$. The $[\mathrm{Fe} / \mathrm{H}]$ values for each star were then determined using Equation (8) and the cubic calibration from Mauro et al. (2014) for the Carretta et al. (2009c) metallicity scale:

$$
\begin{aligned}
{[\mathrm{Fe} / \mathrm{H}]=} & -4.61+1.842\left\langle W^{\prime}\right\rangle-0.4428\left\langle W^{\prime}\right\rangle^{2} \\
& +0.04517\left\langle W^{\prime}\right\rangle^{3} .
\end{aligned}
$$

The individual EWs, $\Sigma \mathrm{EW}$, and $W^{\prime}$ values for all NGC 6273 members are provided in Table 7.

A comparison between the observations of Yong et al. (2016) and our CaT data set revealed 27 stars in common. For this subset, the Yong et al. (2016) $[\mathrm{Fe} / \mathrm{H}]$ values are on average 0.06 dex more metal-rich than ours, but the metallicities from both studies are well-correlated (see Figure 6). Similarly, we found 50 stars in our sample that were observed in both the CaT and Bulge_GC1 setups, and a comparison of the derived $[\mathrm{Fe} / \mathrm{H}]$ values is provided in Figure 6 . The $[\mathrm{Fe} / \mathrm{H}]$ measurements from both data sets are relatively well-correlated, but the $\mathrm{CaT}$ data are 0.12 dex more metal-rich, on average. Therefore, the final CaT-based $[\mathrm{Fe} / \mathrm{H}]$ abundances provided in Table 7, and used throughout the rest of the paper, have been shifted by -0.12 dex in order to place the CaT and Bulge_GC1 data sets on the same scale.

\subsection{Internal Abundance Uncertainties}

For the reasonably high $\mathrm{S} / \mathrm{N}$ Bulge_GC1 region spectra analyzed here, the dominant sources of internal abundance uncertainties are related to the line-to-line abundance scatter from uncertain $\log (g f)$ values, small profile fitting and/or continuum placement errors, and model atmosphere parameter uncertainties. The standard error of the mean provides a reasonable estimate of the abundance errors due to line list and 
Table 5

Chemical Abundances for NGC 6273 Members: $\mathrm{Na}-\mathrm{Cr}$

\begin{tabular}{|c|c|c|c|c|c|c|c|c|c|c|c|c|}
\hline $\begin{array}{l}\text { Star Name } \\
\text { (2MASS) }\end{array}$ & $\begin{array}{l}{[\mathrm{Na} \mathrm{I} / \mathrm{Fe}]} \\
\quad(\mathrm{dex})\end{array}$ & $\begin{array}{c}\Delta[\mathrm{Na} \mathrm{I} / \mathrm{Fe}] \\
\quad(\mathrm{dex})\end{array}$ & $\begin{array}{l}{[\mathrm{Mg} \mathrm{I} / \mathrm{Fe}]} \\
\quad(\mathrm{dex})\end{array}$ & $\begin{array}{c}\Delta[\mathrm{Mg} \mathrm{I} / \mathrm{Fe}] \\
\quad(\mathrm{dex})\end{array}$ & $\begin{array}{c}{[\mathrm{Al} \mathrm{I} / \mathrm{Fe}]} \\
(\mathrm{dex})\end{array}$ & $\begin{array}{l}\Delta[\mathrm{Al} \mathrm{I} / \mathrm{Fe}] \\
\quad(\mathrm{dex})\end{array}$ & $\begin{array}{l}{[\mathrm{Si} \mathrm{I} / \mathrm{Fe}]} \\
\quad(\mathrm{dex})\end{array}$ & $\begin{array}{l}\Delta[\mathrm{Si} \mathrm{I} / \mathrm{Fe}] \\
\quad(\mathrm{dex})\end{array}$ & $\begin{array}{c}{[\mathrm{Ca} \mathrm{I} / \mathrm{Fe}]} \\
(\mathrm{dex})\end{array}$ & $\begin{array}{c}\Delta[\mathrm{Ca} \mathrm{I} / \mathrm{Fe}] \\
\quad(\mathrm{dex})\end{array}$ & $\begin{array}{c}{[\mathrm{Cr} \mathrm{I} / \mathrm{Fe}]} \\
(\mathrm{dex})\end{array}$ & $\begin{array}{c}\Delta[\mathrm{Cr} \text { I } / \mathrm{Fe}] \\
\quad(\mathrm{dex})\end{array}$ \\
\hline $17022227-2613433^{\mathrm{c}}$ & 0.33 & 0.11 & 0.34 & 0.07 & 1.26 & 0.05 & 0.33 & 0.09 & 0.24 & 0.07 & -0.12 & 0.13 \\
\hline $17022817-2616426$ & 0.27 & 0.06 & 0.43 & 0.07 & 0.79 & 0.07 & 0.32 & 0.14 & 0.34 & 0.07 & 0.08 & 0.06 \\
\hline $17022912-2617443^{c}$ & 0.23 & 0.06 & 0.32 & 0.07 & 0.21 & 0.09 & 0.31 & 0.04 & 0.32 & 0.08 & -0.05 & 0.09 \\
\hline $17023087-2618515$ & 0.16 & 0.06 & 0.37 & 0.07 & 0.50 & 0.07 & $\ldots$ & $\ldots$ & 0.21 & 0.09 & $\ldots$ & $\ldots$ \\
\hline $17023192-2614177^{\mathrm{c}}$ & 0.31 & 0.06 & 0.31 & 0.07 & 0.62 & 0.10 & 0.18 & 0.09 & 0.14 & 0.08 & 0.11 & 0.09 \\
\hline $17023225-2614521$ & 0.10 & 0.05 & $\ldots$ & $\ldots$ & 0.11 & 0.07 & 0.23 & 0.13 & 0.15 & 0.07 & -0.11 & 0.09 \\
\hline $17023338-2617104^{c}$ & 0.55 & 0.05 & $\cdots$ & $\cdots$ & 1.01 & 0.07 & 0.27 & 0.10 & 0.29 & 0.08 & 0.27 & 0.09 \\
\hline $17023342-2616165$ & 0.13 & 0.04 & $\cdots$ & $\cdots$ & 0.57 & 0.07 & 0.26 & 0.10 & 0.19 & 0.09 & 0.14 & 0.05 \\
\hline 17023346-2616375 & 0.52 & 0.07 & 0.49 & 0.07 & 1.02 & 0.07 & 0.20 & 0.09 & 0.27 & 0.08 & 0.01 & 0.09 \\
\hline $17023388-2607556$ & 0.17 & 0.06 & 0.40 & 0.07 & 0.38 & 0.07 & 0.29 & 0.06 & 0.32 & 0.07 & 0.16 & 0.09 \\
\hline $17023394-2616196$ & 0.27 & 0.06 & $\ldots$ & $\cdots$ & 0.69 & 0.07 & 0.34 & 0.10 & 0.20 & 0.10 & $\cdots$ & $\cdots$ \\
\hline $17023435-2616386$ & 0.43 & 0.06 & $\cdots$ & $\ldots$ & 1.19 & 0.06 & 0.48 & 0.04 & 0.24 & 0.08 & 0.07 & 0.12 \\
\hline $17023459-2615560^{\mathrm{c}}$ & 0.05 & 0.08 & $\ldots$ & $\ldots$ & 0.81 & 0.07 & 0.41 & 0.01 & 0.32 & 0.07 & -0.02 & 0.06 \\
\hline $17023460-2616038$ & 0.42 & 0.06 & $\ldots$ & $\ldots$ & $\ldots$ & $\ldots$ & 0.39 & 0.09 & 0.24 & 0.07 & 0.20 & 0.06 \\
\hline $17023517-2616130$ & 0.23 & 0.06 & 0.41 & 0.07 & 1.07 & 0.05 & 0.39 & 0.06 & 0.20 & 0.09 & -0.06 & 0.12 \\
\hline $17023523-2617058$ & 0.52 & 0.05 & 0.24 & 0.07 & 0.77 & 0.07 & 0.37 & 0.07 & 0.30 & 0.08 & -0.02 & 0.06 \\
\hline $17023529-2613089^{c}$ & -0.04 & 0.06 & 0.40 & 0.07 & 0.36 & 0.07 & 0.34 & 0.04 & 0.27 & 0.07 & -0.04 & 0.09 \\
\hline $17023551-2616175$ & $\ldots$ & $\ldots$ & $\ldots$ & $\ldots$ & $\ldots$ & $\ldots$ & $\ldots$ & $\ldots$ & $\ldots$ & $\ldots$ & $\ldots$ & $\ldots$ \\
\hline $17023583-2616444$ & 0.31 & 0.09 & $\ldots$ & $\ldots$ & 0.86 & 0.07 & 0.32 & 0.09 & 0.24 & 0.07 & $\cdots$ & $\ldots$ \\
\hline $17023589-2615218$ & 0.42 & 0.11 & 0.43 & 0.07 & 1.04 & 0.05 & 0.23 & 0.04 & 0.19 & 0.08 & $\ldots$ & $\ldots$ \\
\hline $17023595-2615342^{c}$ & 0.62 & 0.05 & 0.24 & 0.07 & 0.89 & 0.07 & 0.24 & 0.10 & 0.41 & 0.09 & 0.24 & 0.05 \\
\hline $17023618-2616576$ & $\cdots$ & $\cdots$ & $\cdots$ & $\cdots$ & $\cdots$ & $\cdots$ & -0.11 & 0.01 & 0.14 & 0.09 & -0.20 & 0.09 \\
\hline $17023685-2616454^{c}$ & 0.50 & 0.06 & $\ldots$ & $\ldots$ & 1.08 & 0.07 & 0.52 & 0.10 & 0.32 & 0.07 & $\ldots$ & $\ldots$ \\
\hline $17023694-2615130$ & -0.03 & 0.06 & $\ldots$ & $\ldots$ & $\ldots$ & $\ldots$ & -0.01 & 0.10 & -0.07 & 0.08 & $\ldots$ & $\cdots$ \\
\hline $17023720-2614581^{\mathrm{a}}$ & 0.36 & 0.04 & 0.21 & 0.07 & 0.93 & 0.07 & 0.46 & 0.09 & 0.23 & 0.09 & $\ldots$ & $\ldots$ \\
\hline $17023723-2617063$ & $\cdots$ & $\cdots$ & 0.31 & 0.07 & 0.68 & 0.07 & 0.30 & 0.07 & 0.15 & 0.07 & -0.10 & 0.14 \\
\hline $17023728-2617024$ & 0.62 & 0.06 & $\cdots$ & $\cdots$ & 0.94 & 0.05 & 0.54 & 0.02 & 0.45 & 0.11 & $\ldots$ & $\ldots$ \\
\hline $17023744-2615306$ & 0.46 & 0.06 & 0.40 & 0.07 & 0.95 & 0.12 & 0.17 & 0.11 & 0.23 & 0.07 & 0.01 & 0.09 \\
\hline $17023783-2615095^{\mathrm{c}}$ & $\cdots$ & $\ldots$ & $\ldots$ & $\ldots$ & $\ldots$ & $\ldots$ & $\ldots$ & $\ldots$ & $\ldots$ & $\ldots$ & $\ldots$ & $\ldots$ \\
\hline $17023898-2618010$ & -0.09 & 0.06 & 0.35 & 0.07 & 0.22 & 0.07 & 0.32 & 0.12 & 0.19 & 0.09 & $\ldots$ & $\ldots$ \\
\hline $17023916-2616500$ & $\cdots$ & $\cdots$ & 0.33 & 0.07 & $\cdots$ & $\cdots$ & 0.24 & 0.10 & 0.26 & 0.08 & 0.00 & 0.10 \\
\hline $17023938-2619361$ & 0.13 & 0.06 & $\cdots$ & $\cdots$ & 0.76 & 0.07 & 0.25 & 0.18 & 0.26 & 0.08 & $\cdots$ & $\cdots$ \\
\hline $17023943-2615343$ & 0.01 & 0.06 & 0.32 & 0.07 & 0.54 & 0.07 & 0.31 & 0.10 & 0.10 & 0.11 & $\cdots$ & $\cdots$ \\
\hline $17023946-2615017^{\mathrm{a}}$ & 0.31 & 0.04 & $\ldots$ & $\ldots$ & 0.75 & 0.07 & 0.15 & 0.04 & 0.08 & 0.08 & 0.08 & 0.09 \\
\hline $17023956-2617202^{\mathrm{c}}$ & 0.26 & 0.06 & $\cdots$ & $\ldots$ & 1.04 & 0.07 & 0.24 & 0.09 & 0.08 & 0.10 & $\ldots$ & $\ldots$ \\
\hline $17023984-2617360^{\mathrm{a}}$ & 0.24 & 0.04 & 0.45 & 0.07 & 0.95 & 0.06 & 0.34 & 0.08 & 0.36 & 0.11 & 0.04 & 0.10 \\
\hline $17023993-2616370^{c}$ & $\ldots$ & $\ldots$ & $\ldots$ & $\ldots$ & $\ldots$ & $\ldots$ & $\ldots$ & $\ldots$ & $\ldots$ & $\ldots$ & $\ldots$ & $\ldots$ \\
\hline $17024016-2615588$ & $\ldots$ & $\ldots$ & $\ldots$ & $\ldots$ & $\ldots$ & $\ldots$ & $\ldots$ & $\ldots$ & $\ldots$ & $\ldots$ & $\ldots$ & $\ldots$ \\
\hline $17024032-2617400$ & 0.59 & 0.06 & 0.31 & 0.07 & 0.89 & 0.05 & 0.11 & 0.18 & 0.16 & 0.09 & $\ldots$ & $\ldots$ \\
\hline $17024041-2617149$ & 0.21 & 0.06 & $\ldots$ & $\ldots$ & $\ldots$ & $\ldots$ & 0.55 & 0.10 & 0.37 & 0.09 & -0.04 & 0.09 \\
\hline $17024104-2616507^{\mathrm{b}}$ & 0.29 & 0.06 & 0.50 & 0.07 & $\cdots$ & $\cdots$ & 0.26 & 0.08 & 0.09 & 0.08 & 0.09 & 0.09 \\
\hline $17024128-2616015$ & $\ldots$ & $\ldots$ & $\ldots$ & $\cdots$ & $\cdots$ & $\cdots$ & $\ldots$ & $\ldots$ & $\cdots$ & $\cdots$ & $\ldots$ & $\cdots$ \\
\hline $17024132-2613517^{\mathrm{a}}$ & $\cdots$ & $\cdots$ & $\cdots$ & $\cdots$ & $\cdots$ & $\cdots$ & $\cdots$ & $\cdots$ & $\cdots$ & $\ldots$ & $\ldots$ & $\ldots$ \\
\hline $17024153-2621081$ & 0.49 & 0.05 & 0.33 & 0.07 & 1.24 & 0.05 & 0.24 & 0.09 & 0.37 & 0.08 & 0.19 & 0.07 \\
\hline $17024165-2617033^{\mathrm{b}}$ & 0.49 & 0.06 & $\ldots$ & $\ldots$ & 0.92 & 0.07 & 0.30 & 0.09 & 0.29 & 0.06 & $\ldots$ & $\ldots$ \\
\hline $17024173-2616245$ & -0.03 & 0.06 & 0.54 & 0.07 & 0.32 & 0.07 & 0.44 & 0.14 & 0.22 & 0.07 & -0.04 & 0.16 \\
\hline $17024226-2615137$ & 0.32 & 0.06 & $\ldots$ & $\ldots$ & $\ldots$ & $\ldots$ & 0.14 & 0.09 & 0.45 & 0.10 & $\ldots$ & $\ldots$ \\
\hline
\end{tabular}


Table 5

(Continued)

\begin{tabular}{|c|c|c|c|c|c|c|c|c|c|c|c|c|}
\hline $\begin{array}{l}\text { Star Name } \\
\text { (2MASS) }\end{array}$ & $\begin{array}{l}{[\mathrm{Na} \mathrm{I} / \mathrm{Fe}]} \\
\quad(\mathrm{dex})\end{array}$ & $\begin{array}{l}\Delta[\mathrm{Na} \mathrm{I} / \mathrm{Fe}] \\
\quad(\mathrm{dex})\end{array}$ & $\begin{array}{l}{[\mathrm{Mg} \mathrm{I} / \mathrm{Fe}]} \\
\quad(\mathrm{dex})\end{array}$ & $\begin{array}{l}\Delta[\mathrm{Mg} \mathrm{I} / \mathrm{Fe}] \\
\quad(\mathrm{dex})\end{array}$ & $\begin{array}{c}{[\mathrm{Al} \mathrm{I} / \mathrm{Fe}]} \\
(\mathrm{dex})\end{array}$ & $\begin{array}{l}\Delta[\mathrm{Al} / \mathrm{I} / \mathrm{Fe}] \\
\quad(\mathrm{dex})\end{array}$ & $\begin{array}{l}{[\mathrm{Si} \text { I/Fe] }} \\
\quad(\mathrm{dex})\end{array}$ & $\begin{array}{l}\Delta[\mathrm{Si} \mathrm{I} / \mathrm{Fe}] \\
\quad(\mathrm{dex})\end{array}$ & $\begin{array}{c}{[\mathrm{Ca} \mathrm{I} / \mathrm{Fe}]} \\
(\mathrm{dex})\end{array}$ & $\begin{array}{c}\Delta[\mathrm{Ca} \mathrm{I} / \mathrm{Fe}] \\
\quad(\mathrm{dex})\end{array}$ & $\begin{array}{l}{[\mathrm{Cr} \mathrm{I} / \mathrm{Fe}]} \\
(\mathrm{dex})\end{array}$ & $\begin{array}{c}\Delta[\mathrm{Cr} \mathrm{I} / \mathrm{Fe}] \\
\quad(\mathrm{dex})\end{array}$ \\
\hline $17024242-2615557$ & 0.08 & 0.08 & 0.32 & 0.07 & 0.76 & 0.07 & 0.17 & 0.06 & 0.15 & 0.07 & 0.06 & 0.09 \\
\hline $17024289-2615274^{a}$ & 0.21 & 0.04 & $\ldots$ & $\ldots$ & 0.71 & 0.07 & 0.34 & 0.06 & 0.14 & 0.08 & $\ldots$ & $\ldots$ \\
\hline $17024371-2620183^{\mathrm{a}}$ & 0.52 & 0.12 & 0.50 & 0.07 & 1.03 & 0.07 & 0.25 & 0.13 & 0.17 & 0.07 & -0.05 & 0.09 \\
\hline $17024377-2615526^{c}$ & 0.50 & 0.06 & 0.24 & 0.07 & 0.83 & 0.05 & 0.33 & 0.15 & 0.30 & 0.08 & 0.05 & 0.09 \\
\hline $17024412-2616495$ & 0.19 & 0.11 & $\ldots$ & $\ldots$ & 0.75 & 0.07 & 0.45 & 0.10 & 0.27 & 0.09 & 0.12 & 0.05 \\
\hline $17024416-2615177^{\mathrm{b}}$ & 0.12 & 0.06 & $\ldots$ & $\ldots$ & 0.44 & 0.06 & -0.19 & 0.10 & -0.04 & 0.09 & $\ldots$ & $\ldots$ \\
\hline $17024472-2615190$ & $\ldots$ & $\ldots$ & $\ldots$ & $\ldots$ & $\ldots$ & $\ldots$ & $\ldots$ & $\ldots$ & $\ldots$ & $\ldots$ & $\ldots$ & $\ldots$ \\
\hline $17024566-2615124^{\mathrm{a}}$ & -0.16 & 0.06 & $\ldots$ & $\ldots$ & 0.47 & 0.07 & -0.06 & 0.10 & -0.03 & 0.11 & 0.06 & 0.09 \\
\hline $17024625-2610100$ & 0.64 & 0.06 & $\ldots$ & $\ldots$ & 0.98 & 0.07 & 0.45 & 0.05 & 0.37 & 0.07 & -0.11 & 0.09 \\
\hline $17024627-2614484^{c}$ & $\ldots$ & $\ldots$ & $\ldots$ & $\ldots$ & $\ldots$ & $\ldots$ & $\ldots$ & $\ldots$ & $\ldots$ & $\ldots$ & $\ldots$ & $\ldots$ \\
\hline $17024838-2615546$ & -0.08 & 0.05 & 0.48 & 0.07 & 0.37 & 0.07 & 0.12 & 0.09 & 0.33 & 0.09 & -0.12 & 0.13 \\
\hline $17025033-2615582^{\mathrm{a}}$ & 0.28 & 0.09 & 0.29 & 0.07 & 0.58 & 0.07 & 0.35 & 0.06 & 0.27 & 0.09 & 0.06 & 0.06 \\
\hline
\end{tabular}

Notes.

${ }^{a}$ Observed in Johnson et al. (2015b), the Bulge_GC1 setup, and the Calcium Triplet setup.

${ }^{\mathrm{b}}$ Observed in Johnson et al. (2015b) and the Bulge_GC1 setup.

${ }^{c}$ Observed in Johnson et al. (2015b) and the Calcium Triplet setup.

(This table is available in machine-readable form.) 
Table 6

Chemical Abundances for NGC 6273 Members: $\mathrm{Fe}-\mathrm{Eu}$

\begin{tabular}{|c|c|c|c|c|c|c|c|c|c|c|}
\hline $\begin{array}{l}\text { Star Name } \\
\text { (2MASS) }\end{array}$ & $\begin{array}{l}{[\mathrm{Fe} \mathrm{I} / \mathrm{H}]} \\
(\mathrm{dex})\end{array}$ & $\begin{array}{l}\Delta[\mathrm{Fe} \mathrm{I} / \mathrm{H}] \\
\quad(\mathrm{dex})\end{array}$ & $\begin{array}{c}{[\mathrm{Fe} \text { II/H }]} \\
(\mathrm{dex})\end{array}$ & $\begin{array}{c}\Delta[\mathrm{Fe} \text { II } / \mathrm{H}] \\
\quad(\operatorname{dex})\end{array}$ & $\begin{array}{l}{[\mathrm{Ni} \mathrm{I} / \mathrm{Fe}]} \\
(\mathrm{dex})\end{array}$ & $\begin{array}{c}\Delta[\mathrm{Ni} \mathrm{I} / \mathrm{Fe}] \\
(\mathrm{dex})\end{array}$ & $\begin{array}{l}{[\mathrm{La} \mathrm{II} / \mathrm{Fe}]} \\
(\mathrm{dex})\end{array}$ & $\begin{array}{c}\Delta[\mathrm{La} \mathrm{II} / \mathrm{Fe}] \\
(\mathrm{dex})\end{array}$ & $\begin{array}{l}{[\mathrm{Eu} \text { II } / \mathrm{Fe}]} \\
\quad(\mathrm{dex})\end{array}$ & $\begin{array}{c}\Delta[\mathrm{Eu} \mathrm{II} / \mathrm{Fe}] \\
(\mathrm{dex})\end{array}$ \\
\hline $17022227-2613433^{c}$ & -1.67 & 0.07 & -1.67 & 0.08 & -0.03 & 0.07 & 0.12 & 0.08 & 0.41 & 0.07 \\
\hline $17022817-2616426$ & -1.66 & 0.07 & -1.66 & 0.08 & 0.07 & 0.07 & 0.28 & 0.08 & 0.14 & 0.10 \\
\hline $17022912-2617443^{\mathrm{c}}$ & -1.80 & 0.07 & -1.80 & 0.08 & -0.02 & 0.08 & 0.57 & 0.06 & 0.34 & 0.10 \\
\hline $17023087-2618515$ & -1.85 & 0.07 & -1.86 & 0.06 & -0.03 & 0.07 & 0.10 & 0.08 & 0.66 & 0.10 \\
\hline $17023192-2614177^{\mathrm{c}}$ & -1.48 & 0.07 & -1.49 & 0.08 & -0.13 & 0.08 & 1.17 & 0.13 & 0.38 & 0.10 \\
\hline $17023225-2614521$ & -1.71 & 0.07 & -1.71 & 0.07 & -0.09 & 0.07 & 0.35 & 0.08 & 0.62 & 0.10 \\
\hline $17023338-2617104^{\mathrm{c}}$ & -1.63 & 0.07 & -1.63 & 0.09 & 0.06 & 0.07 & 0.64 & 0.06 & 0.44 & 0.10 \\
\hline $17023342-2616165$ & -1.51 & 0.07 & -1.50 & 0.08 & -0.135 & 0.06 & 0.36 & 0.08 & 0.21 & 0.10 \\
\hline $17023346-2616375$ & -1.42 & 0.07 & -1.41 & 0.10 & -0.05 & 0.07 & 0.67 & 0.06 & 0.58 & 0.09 \\
\hline $17023388-2607556$ & -1.78 & 0.07 & -1.78 & 0.07 & -0.12 & 0.09 & 0.04 & 0.08 & 0.56 & 0.10 \\
\hline $17023394-2616196$ & -1.94 & 0.07 & -1.93 & 0.07 & $\ldots$ & $\cdots$ & 0.07 & 0.08 & $\cdots$ & $\cdots$ \\
\hline $17023435-2616386$ & -1.77 & 0.07 & -1.77 & 0.07 & 0.01 & 0.06 & -0.11 & 0.08 & 0.25 & 0.07 \\
\hline $17023459-2615560^{\mathrm{c}}$ & -1.85 & 0.07 & -1.85 & 0.08 & -0.02 & 0.07 & 0.34 & 0.08 & 0.13 & 0.10 \\
\hline $17023460-2616038$ & -1.72 & 0.07 & -1.72 & 0.09 & 0.03 & 0.08 & 0.23 & 0.08 & $\ldots$ & $\ldots$ \\
\hline $17023517-2616130$ & -1.39 & 0.07 & -1.39 & 0.09 & -0.17 & 0.10 & 0.77 & 0.08 & 0.35 & 0.10 \\
\hline $17023523-2617058$ & -1.52 & 0.07 & -1.52 & 0.09 & -0.01 & 0.07 & 0.54 & 0.08 & 0.28 & 0.10 \\
\hline $17023529-2613089^{c}$ & -1.63 & 0.07 & -1.63 & 0.08 & -0.04 & 0.06 & 0.50 & 0.08 & 0.29 & 0.10 \\
\hline $17023551-2616175$ & $\cdots$ & $\cdots$ & $\ldots$ & $\cdots$ & $\ldots$ & $\cdots$ & $\ldots$ & $\cdots$ & $\cdots$ & $\cdots$ \\
\hline $17023583-2616444$ & -1.70 & 0.08 & -1.70 & 0.10 & -0.10 & 0.15 & $\cdots$ & $\cdots$ & $\cdots$ & $\cdots$ \\
\hline 17023589-2615218 & -1.55 & 0.07 & -1.57 & 0.10 & 0.00 & 0.07 & 0.70 & 0.08 & 0.42 & 0.10 \\
\hline $17023595-2615342^{\mathrm{c}}$ & -1.22 & 0.07 & -1.22 & 0.09 & 0.08 & 0.09 & 0.93 & 0.08 & 0.27 & 0.10 \\
\hline $17023618-2616576$ & -1.55 & 0.07 & -1.55 & 0.07 & -0.01 & 0.09 & -0.12 & 0.18 & 0.24 & 0.10 \\
\hline $17023685-2616454^{\mathrm{c}}$ & -1.77 & 0.07 & -1.79 & 0.08 & 0.18 & 0.06 & 0.25 & 0.07 & 0.47 & 0.10 \\
\hline $17023694-2615130$ & -1.48 & 0.08 & $\ldots$ & $\cdots$ & -0.17 & 0.07 & 0.51 & 0.08 & 0.71 & 0.10 \\
\hline $17023720-2614581^{\mathrm{a}}$ & -1.53 & 0.08 & -1.54 & 0.08 & -0.10 & 0.07 & 0.37 & 0.08 & 0.37 & 0.10 \\
\hline $17023723-2617063$ & -1.64 & 0.07 & -1.63 & 0.09 & -0.05 & 0.06 & 0.12 & 0.06 & 0.19 & 0.10 \\
\hline $17023728-2617024$ & -1.83 & 0.07 & $\ldots$ & $\cdots$ & -0.04 & 0.07 & -0.24 & 0.08 & 0.32 & 0.07 \\
\hline 17023744-2615306 & -1.78 & 0.08 & -1.78 & 0.10 & 0.11 & 0.07 & 0.10 & 0.08 & 0.62 & 0.10 \\
\hline $17023783-2615095^{\mathrm{c}}$ & $\ldots$ & $\ldots$ & $\ldots$ & $\ldots$ & $\ldots$ & $\cdots$ & $\ldots$ & $\cdots$ & $\ldots$ & $\ldots$ \\
\hline $17023898-2618010$ & -1.47 & 0.07 & -1.49 & 0.10 & $\cdots$ & $\cdots$ & 0.41 & 0.08 & 0.45 & 0.10 \\
\hline $17023916-2616500$ & -1.70 & 0.07 & -1.71 & 0.08 & -0.09 & 0.06 & -0.03 & 0.08 & 0.24 & 0.10 \\
\hline $17023938-2619361$ & -1.71 & 0.07 & -1.71 & 0.08 & -0.04 & 0.14 & -0.38 & 0.08 & 0.39 & 0.10 \\
\hline $17023943-2615343$ & -1.69 & 0.08 & -1.70 & 0.08 & -0.14 & 0.07 & 0.08 & 0.08 & 0.16 & 0.10 \\
\hline $17023946-2615017^{\mathrm{a}}$ & -1.48 & 0.07 & -1.49 & 0.08 & 0.13 & 0.05 & -0.06 & 0.08 & 0.19 & 0.10 \\
\hline $17023956-2617202^{\mathrm{c}}$ & -1.45 & 0.07 & -1.44 & 0.13 & $\ldots$ & $\ldots$ & 0.70 & 0.08 & 0.30 & 0.10 \\
\hline $17023984-2617360^{\mathrm{a}}$ & -1.40 & 0.07 & -1.42 & 0.09 & -0.16 & 0.06 & 0.57 & 0.12 & 0.41 & 0.10 \\
\hline $17023993-2616370^{\mathrm{c}}$ & $\cdots$ & $\cdots$ & $\cdots$ & $\cdots$ & $\ldots$ & $\cdots$ & $\cdots$ & $\cdots$ & $\cdots$ & $\cdots$ \\
\hline $17024016-2615588$ & $\cdots$ & $\cdots$ & $\cdots$ & $\cdots$ & $\ldots$ & $\cdots$ & $\cdots$ & $\cdots$ & $\cdots$ & $\cdots$ \\
\hline $17024032-2617400$ & -1.43 & 0.07 & -1.44 & 0.08 & -0.15 & 0.06 & 0.23 & 0.08 & 0.47 & 0.11 \\
\hline $17024041-2617149$ & -1.70 & 0.07 & -1.70 & 0.12 & 0.03 & 0.10 & 0.72 & 0.07 & 0.18 & 0.10 \\
\hline $17024104-2616507^{\mathrm{b}}$ & -1.74 & 0.07 & -1.73 & 0.09 & -0.13 & 0.06 & 0.05 & 0.08 & 0.44 & 0.10 \\
\hline $17024128-2616015$ & $\ldots$ & $\cdots$ & $\ldots$ & $\cdots$ & $\ldots$ & $\cdots$ & $\cdots$ & $\cdots$ & $\ldots$ & $\ldots$ \\
\hline $17024132-2613517^{\mathrm{a}}$ & $\ldots$ & $\cdots$ & $\ldots$ & $\ldots$ & $\ldots$ & $\cdots$ & $\ldots$ & $\ldots$ & $\ldots$ & $\ldots$ \\
\hline $17024153-2621081$ & -1.53 & 0.07 & -1.52 & 0.09 & -0.08 & 0.08 & 0.76 & 0.08 & 0.26 & 0.10 \\
\hline $17024165-2617033^{b}$ & -1.90 & 0.08 & -1.90 & 0.07 & -0.04 & 0.08 & -0.08 & 0.08 & 0.63 & 0.10 \\
\hline $17024173-2616245$ & -1.58 & 0.07 & -1.56 & 0.08 & -0.10 & 0.09 & 0.69 & 0.08 & 0.46 & 0.08 \\
\hline $17024226-2615137$ & -1.59 & 0.08 & $\cdots$ & $\cdots$ & $\ldots$ & $\cdots$ & $\cdots$ & $\cdots$ & 0.34 & 0.10 \\
\hline $17024242-2615557$ & -1.59 & 0.07 & -1.60 & 0.08 & -0.09 & 0.07 & 0.04 & 0.08 & 0.50 & 0.10 \\
\hline $17024289-2615274^{\mathrm{a}}$ & -1.71 & 0.08 & -1.68 & 0.12 & -0.07 & 0.10 & $\ldots$ & $\ldots$ & 0.32 & 0.10 \\
\hline $17024371-2620183^{a}$ & -1.73 & 0.07 & -1.72 & 0.09 & -0.24 & 0.08 & 0.32 & 0.08 & $\cdots$ & $\cdots$ \\
\hline $17024377-2615526^{\mathrm{c}}$ & -1.42 & 0.07 & -1.42 & 0.14 & 0.03 & 0.08 & 0.78 & 0.07 & 0.23 & 0.08 \\
\hline $17024412-2616495$ & -1.52 & 0.07 & -1.50 & 0.07 & -0.08 & 0.10 & 0.61 & 0.06 & 0.31 & 0.10 \\
\hline $17024416-2615177^{\mathrm{b}}$ & -1.43 & 0.08 & -1.45 & 0.07 & -0.35 & 0.07 & 0.07 & 0.10 & $\cdots$ & $\cdots$ \\
\hline $17024472-2615190$ & $\ldots$ & $\cdots$ & $\ldots$ & $\cdots$ & $\ldots$ & $\cdots$ & $\cdots$ & $\cdots$ & $\ldots$ & $\cdots$ \\
\hline $17024566-2615124^{a}$ & -1.08 & 0.08 & -1.10 & 0.08 & -0.21 & 0.07 & 0.64 & 0.08 & 0.66 & 0.10 \\
\hline $17024625-2610100$ & -2.01 & 0.07 & -1.99 & 0.07 & 0.07 & 0.08 & $\cdots$ & $\cdots$ & 0.35 & 0.10 \\
\hline $17024627-2614484^{\mathrm{c}}$ & $\ldots$ & $\ldots$ & $\ldots$ & $\ldots$ & $\ldots$ & $\ldots$ & $\ldots$ & $\ldots$ & $\ldots$ & $\ldots$ \\
\hline $17024838-2615546$ & -1.53 & 0.07 & -1.54 & 0.09 & -0.13 & 0.07 & 0.41 & 0.08 & 0.37 & 0.10 \\
\hline $17025033-2615582^{a}$ & -1.26 & 0.07 & -1.27 & 0.08 & 0.04 & 0.07 & 0.73 & 0.08 & 0.52 & 0.10 \\
\hline
\end{tabular}

Notes.

${ }^{\text {a }}$ Observed in Johnson et al. (2015b), the Bulge_GC1 setup, and the Calcium Triplet setup.

${ }^{\mathrm{b}}$ Observed in Johnson et al. (2015b) and the Bulge_GC1 setup.

${ }^{c}$ Observed in Johnson et al. (2015b) and the Calcium Triplet setup.

(This table is available in machine-readable form.)

profile fitting uncertainties, and for this data set we find a typical measurement uncertainty of $0.05 \operatorname{dex}(\sigma=0.03 \mathrm{dex})$ in $\log \epsilon(X)$.
In order to estimate the uncertainties in $T_{\text {eff }}$ and $\log (g)$, we provide a comparison of the spectroscopically derived parameters with those expected from Dartmouth isochrones 
Table 7

Calcium Triplet Metallicity Data

\begin{tabular}{|c|c|c|c|c|c|c|}
\hline $\begin{array}{l}\text { Star Name } \\
\text { (2MASS) }\end{array}$ & $\begin{array}{c}\mathrm{EW}_{(\AA 542} \\
(\AA)\end{array}$ & $\begin{array}{c}\mathrm{EW}_{88662} \\
(\AA)\end{array}$ & $\underset{(\AA ̊)}{\sum \underset{(\mathrm{EW}}{\mathrm{E}}}$ & $\begin{array}{l}W^{\prime} \\
(\AA)\end{array}$ & $\begin{array}{c}{[\mathrm{Fe} / \mathrm{H}]} \\
(\mathrm{dex})\end{array}$ & $\begin{array}{c}\Delta[\mathrm{Fe} / \mathrm{H}] \\
(\mathrm{dex})\end{array}$ \\
\hline \multicolumn{7}{|c|}{ M2FS Calcium Triplet Members } \\
\hline $17021380-2613223$ & 1.99 & 1.45 & 3.44 & 3.17 & -2.01 & 0.10 \\
\hline $17021778-2616058$ & 2.11 & 1.47 & 3.58 & 3.21 & -1.99 & 0.12 \\
\hline $17022040-2616289^{\mathrm{b}}$ & 2.54 & 1.77 & 4.31 & 3.50 & -1.89 & 0.13 \\
\hline $17022413-2619124$ & 2.37 & 1.73 & 4.10 & 3.77 & -1.79 & 0.10 \\
\hline $17022442-2616495$ & 3.29 & 2.46 & 5.75 & 4.47 & -1.51 & 0.10 \\
\hline
\end{tabular}

Notes.

a Observed in Johnson et al. (2015b), the Bulge_GC1 setup, and the M2FS Calcium Triplet setup.

${ }^{\mathrm{b}}$ Observed in Johnson et al. (2015b) and the M2FS Calcium Triplet setup.

${ }^{c}$ Observed in the Bulge_GC1 and M2FS Calcium Triplet setups.

${ }^{\mathrm{d}}$ Observed in the M2FS Calcium Triplet and FLAMES Calcium Triplet setups.

(This table is available in its entirety in machine-readable form.)
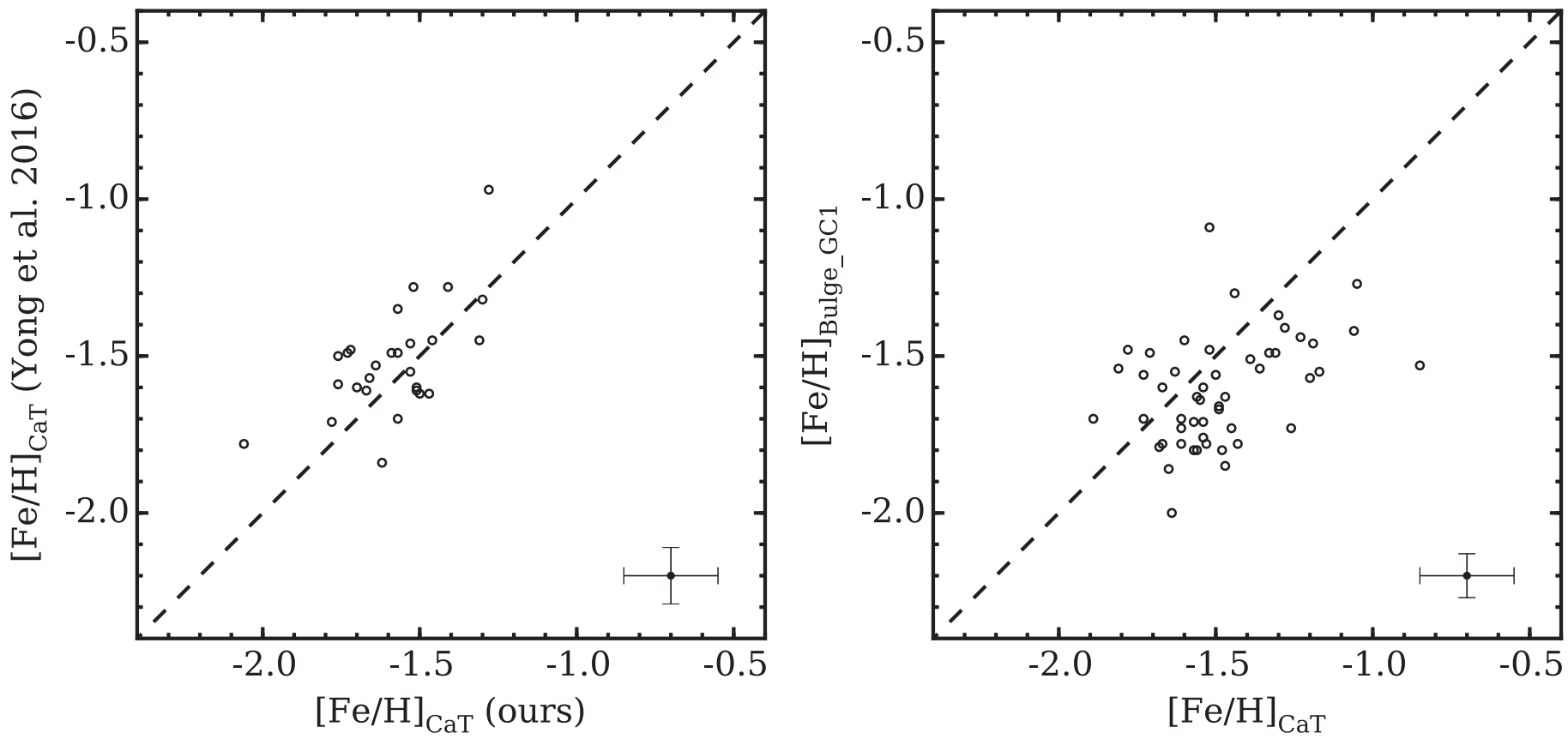

Figure 6. Left: a comparison of the $\mathrm{CaT}[\mathrm{Fe} / \mathrm{H}]$ values derived in this work and Yong et al. (2016), for 27 stars in common. The dashed line indicates perfect agreement. Right: a comparison of the $[\mathrm{Fe} / \mathrm{H}]$ values derived from the $\mathrm{CaT}$ and Bulge_GC1 data sets of this work, for 50 stars in common. Note that in both panels our $\mathrm{CaT}[\mathrm{Fe} / \mathrm{H}]$ values are those derived from Equation (9) and have not yet been corrected to place the $\mathrm{CaT}[\mathrm{Fe} / \mathrm{H}]$ abundances on the Bulge_GC1 [Fe/H] scale. Typical error bars are shown in the bottom right corner of each panel.

(Dotter et al. 2008) with ages of $12 \mathrm{Gyr},[\alpha / \mathrm{Fe}]=+0.4 \mathrm{dex}$, and $[\mathrm{Fe} / \mathrm{H}]=-1.75,-1.50$, and $-1.20 \mathrm{dex}$ in Figure 7 . The isochrones with different $[\mathrm{Fe} / \mathrm{H}]$ are included because of the metallicity spread detected in the cluster (Han et al. 2015; Johnson et al. 2015b; Yong et al. 2016; see also Section 5.1). Figure 7 shows that the derived temperature and surface gravity values are in good agreement with those predicted by the isochrones. Specifically, we find the average differences between the spectroscopic and isochrone temperature $\left(\Delta T_{\text {eff }}\right)$ and surface gravity $(\Delta \log (g))$ values to be $-8 \mathrm{~K}$ and $+0.01 \mathrm{cgs}$, respectively, and do not detect any significant trends as a function of temperature, gravity, or metallicity. The dispersions in $\Delta T_{\text {eff }}$ and $\Delta \log (g)$ are found to be $92 \mathrm{~K}$ and
$0.17 \mathrm{cgs}$, respectively. Therefore, we have adopted $100 \mathrm{~K}$ and $0.15 \mathrm{cgs}$ as the typical model atmosphere uncertainties for $T_{\text {eff }}$ and $\log (g)$. For the model atmosphere metallicity, we have adopted an uncertainty of $0.10 \mathrm{dex}$ based on the combined measurement errors of $[\mathrm{Fe} \mathrm{I} / \mathrm{H}]$ and $[\mathrm{Fe} \mathrm{II} / \mathrm{H}]$. Additionally, we estimate the typical $\xi_{\text {mic. }}$ uncertainty to be $0.10 \mathrm{~km} \mathrm{~s}^{-1}$ based on the scatter and fitting uncertainties present in plots of $\log \epsilon(\mathrm{Fe} \mathrm{I})$ versus $\log (\mathrm{EW} / \lambda)$.

The abundance uncertainty values $(\Delta[\mathrm{X} / \mathrm{Fe}]$ or $\Delta[\mathrm{Fe} / \mathrm{H}])$ were determined by rerunning MOOG and changing each model atmosphere parameter by the estimated uncertainties listed previously. Only one parameter was changed per run while the other values were held fixed. To speed up the 

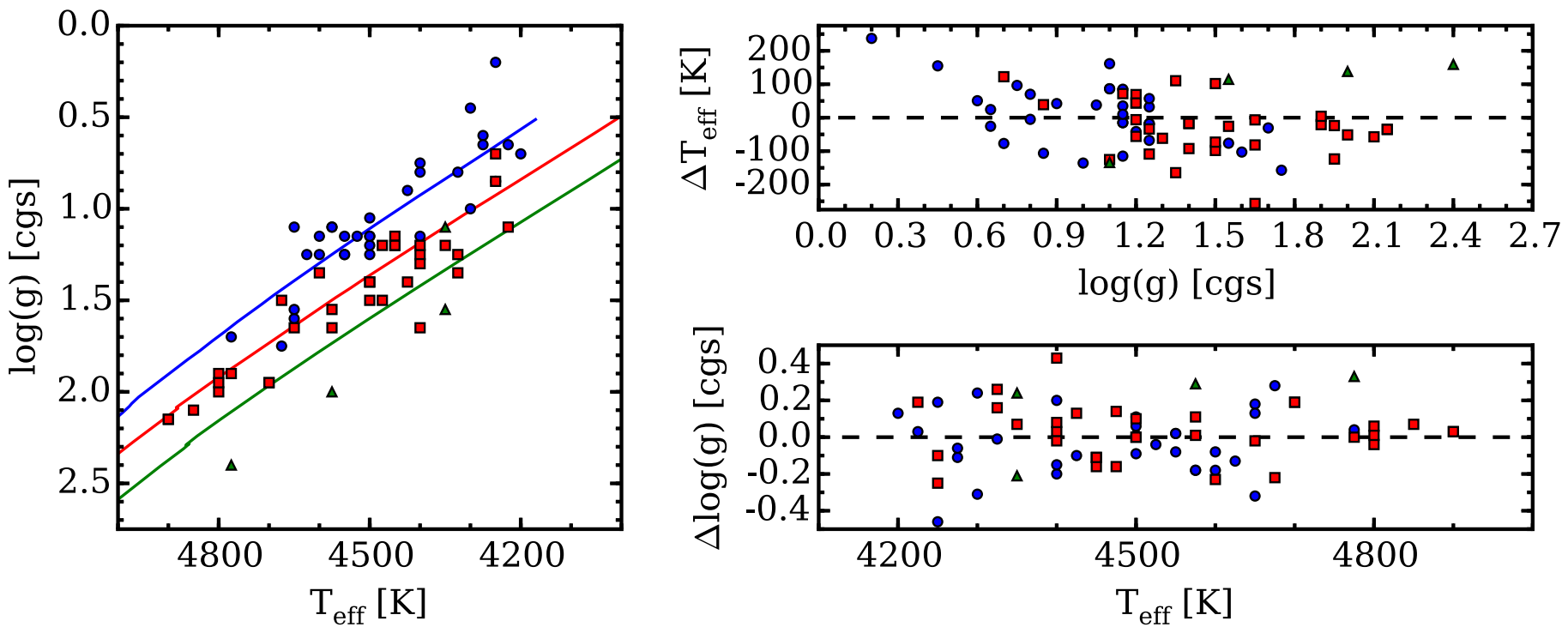

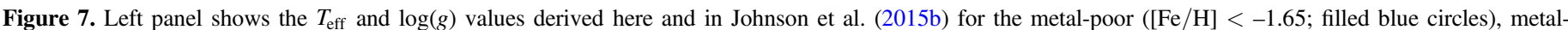

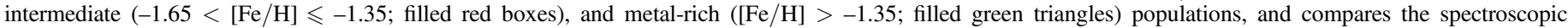

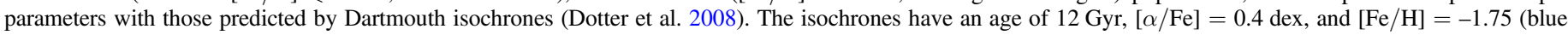

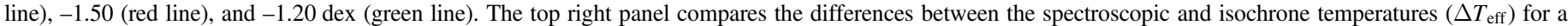

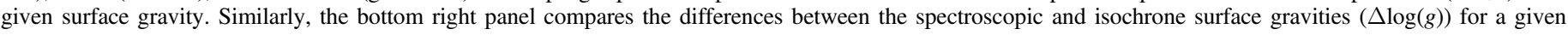
temperature.

analysis, we converted abundances to EWs for the elements measured by spectrum synthesis using the ewfind driver in MOOG. The total internal abundance uncertainties listed in Tables 5-6 were determined by adding the model atmosphere error terms, plus the random measurement uncertainties, in quadrature.

For the CaT data, we estimated the abundance uncertainties by analyzing the correlation between the 8542 and $8662 \AA$ EWs. In other words, we used the strong correlation between $\mathrm{EW}_{8542}$ and $\mathrm{EW}_{8662}$ to predict the $\mathrm{EW}$ of each line based on the other one. The predicted EWs were then propagated through Equations (8)-(9), and the difference between these values and the $[\mathrm{Fe} / \mathrm{H}]$ abundance given in Table 7 was taken as the measurement error. Using this method, we found an average $\Delta[\mathrm{Fe} / \mathrm{H}]=0.15 \mathrm{dex}(\sigma=0.07 \mathrm{dex})$. We note that this value is similar to the fitting uncertainty of Equation (9) (Mauro et al. 2014). A typical $[\mathrm{Fe} / \mathrm{H}]$ uncertainty of 0.15 dex is also similar to the $1 \sigma$ scatter $(0.21 \mathrm{dex})$ between $[\mathrm{Fe} / \mathrm{H}]$ values determined from the CaT and Bulge_GC1 data.

\section{Results and Discussion}

\subsection{Metallicity Distribution}

The color and CaT abundance spreads observed by Piotto et al. (1999) and Rutledge et al. (1997) provided some of the first evidence that NGC 6273 may host stars with different metallicities. More recently, high-resolution spectroscopic measurements from Johnson et al. (2015b) showed that the cluster contains stars with $[\mathrm{Fe} / \mathrm{H}]$ ranging from -1.80 to $-1.30 \mathrm{dex}$, and also found that the cluster hosts at least two distinct populations separated in $[\mathrm{Fe} / \mathrm{H}]$ by $\sim 0.25 \mathrm{dex}$. Similarly, Han et al. (2015) used narrow-band $h k$ photometry to clearly show that the cluster's sub-giant branch and RGB are split into two sequences with different compositions. Yong et al. (2016) also reported $\mathrm{CaT}$ metallicities ranging from $[\mathrm{Fe} / \mathrm{H}]=-1.84$ to $-0.70 \mathrm{dex}$, further indicating the presence of a large metallicity spread in the cluster.
The Johnson et al. (2015b) and Yong et al. (2016) spectroscopic results are based on the analysis of only 18 and 44 RGB stars observed in the Bulge_GC1 and CaT spectral regions, respectively. Therefore, we add here $[\mathrm{Fe} / \mathrm{H}]$ measurements for $51 \mathrm{RGB}$ members in the Bulge_GC1 region and 191 RGB members in the CaT region (see Tables 4, 6, and 7). For the Bulge_GC1 data, we find a full range of $[\mathrm{Fe} / \mathrm{H}]=-2.00$ to -1.09 dex, an average $\langle[\mathrm{Fe} / \mathrm{H}]\rangle=-1.61 \mathrm{dex}$, a dispersion $\left(\sigma_{[\mathrm{Fe} / \mathrm{H}]}\right)$ of $0.18 \mathrm{dex}$, and an interquartile range (IQR) of 0.24 dex. Similarly, the CaT data exhibit a full range of $[\mathrm{Fe} / \mathrm{H}]=-2.22$ to $-0.56 \mathrm{dex}$, an average $\langle[\mathrm{Fe} / \mathrm{H}]\rangle=-1.73 \mathrm{dex}, \sigma_{[\mathrm{Fe} / \mathrm{H}]}=0.24 \mathrm{dex}$, and an IQR of 0.27 dex. A comparison between the Bulge_GC1 and CaT metallicity distributions is shown in Figure 8, and both data sets provide evidence that NGC 6273 harbors an intrinsic metallicity spread.

Further examination of Figure 8 also indicates that NGC 6273 may host distinct populations with different $[\mathrm{Fe} / \mathrm{H}]$, rather than just a broadened distribution. Specifically, Figure 8 suggests that at least three major components may exist: (1) a "metal-poor" population ([Fe/H] $\leqslant-1.65)$; (2) a "metalintermediate" population $(-1.65<[\mathrm{Fe} / \mathrm{H}] \leqslant-1.35)$; and a "metal-rich" tail $([\mathrm{Fe} / \mathrm{H}]>-1.35)$, and that these components constitute $46 \% \pm 8 \%, 48 \% \pm 8 \%$, and $6 \% \pm 4 \%$ of our total Bulge_GC1 data set, respectively. We find the average metallicities of the metal-poor, metal-intermediate, and metal-rich populations to be $\langle[\mathrm{Fe} / \mathrm{H}]\rangle=-1.77 \mathrm{dex}$ $(\sigma=0.08 \mathrm{dex}),\langle[\mathrm{Fe} / \mathrm{H}]\rangle=-1.51 \mathrm{dex}(\sigma=0.07 \mathrm{dex})$, and $\langle[\mathrm{Fe} / \mathrm{H}]\rangle=-1.22 \mathrm{dex} \quad(\sigma=0.09 \mathrm{dex}), \quad$ respectively. The clustering of stars near $[\mathrm{Fe} / \mathrm{H}]=-1.75$ and -1.50 is consistent with the $[\mathrm{Fe} / \mathrm{H}]$ abundances and split RGB sequences derived by Johnson et al. (2015b) and Han et al. (2015), and the presence of a metal-rich tail extending up to at least $[\mathrm{Fe} / \mathrm{H}] \approx-1$ matches the findings of Yong et al. (2016).

Figure 9 indicates that a radial metallicity gradient may exist in the cluster such that the metal-intermediate stars are more centrally concentrated than the metal-poor stars. Although the metal-rich stars observed with the Bulge_GC1 setup all reside 

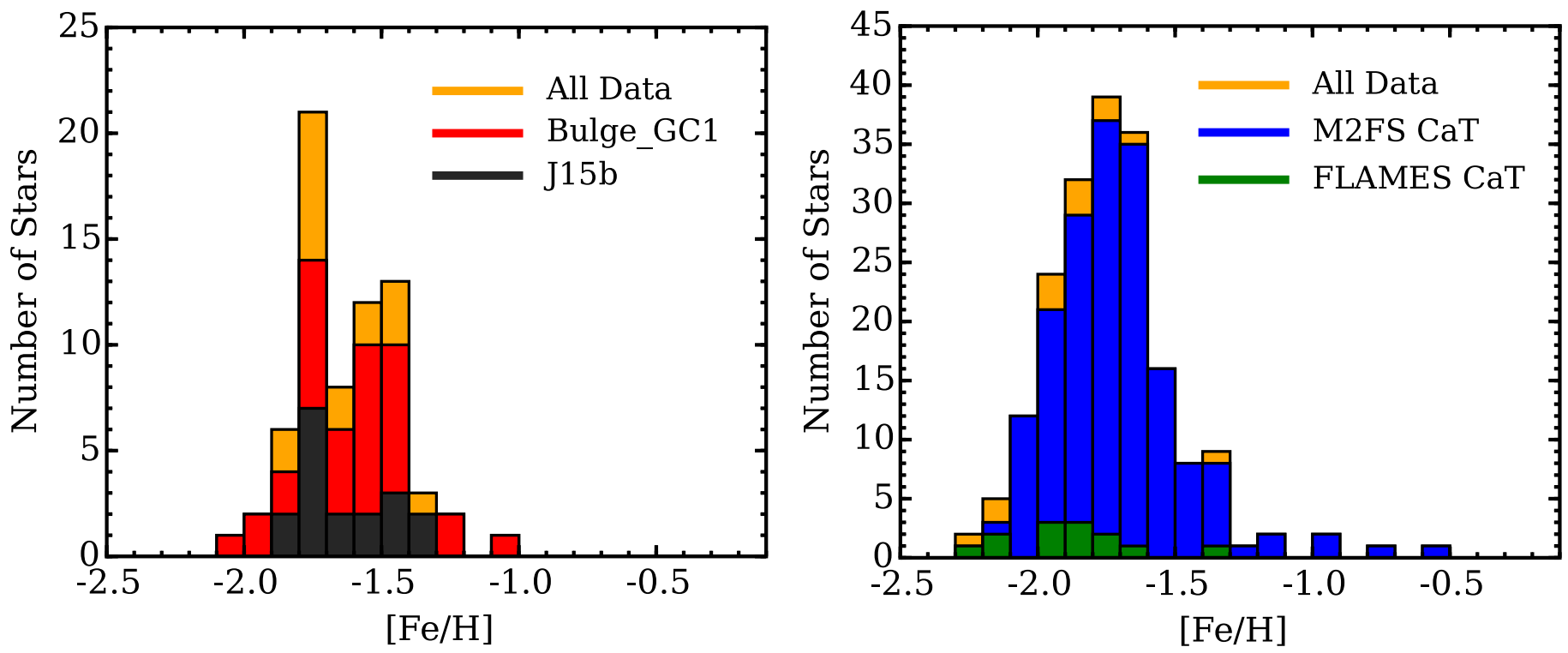

Figure 8. Left and right panels compare the $[\mathrm{Fe} / \mathrm{H}]$ distribution functions derived from data obtained with the Bulge_GC1 and CaT spectrograph setups, respectively For the left panel, the orange histogram represents the sum of the metallicities derived from this work and Johnson et al. (2015b). Similarly, in the right panel, the orange histogram represents the sum of the M2FS and FLAMES CaT metallicities. All of the data are sampled with $0.10 \mathrm{dex}[\mathrm{Fe} / \mathrm{H}]$ bins. Note the broad [Fe/H] range found in both data sets, and also the likely presence of more than one distinct population in the Bulge_GC1 data set. The $[\mathrm{Fe} / \mathrm{H}]$ distributions in both panels only include stars that are radial velocity members.
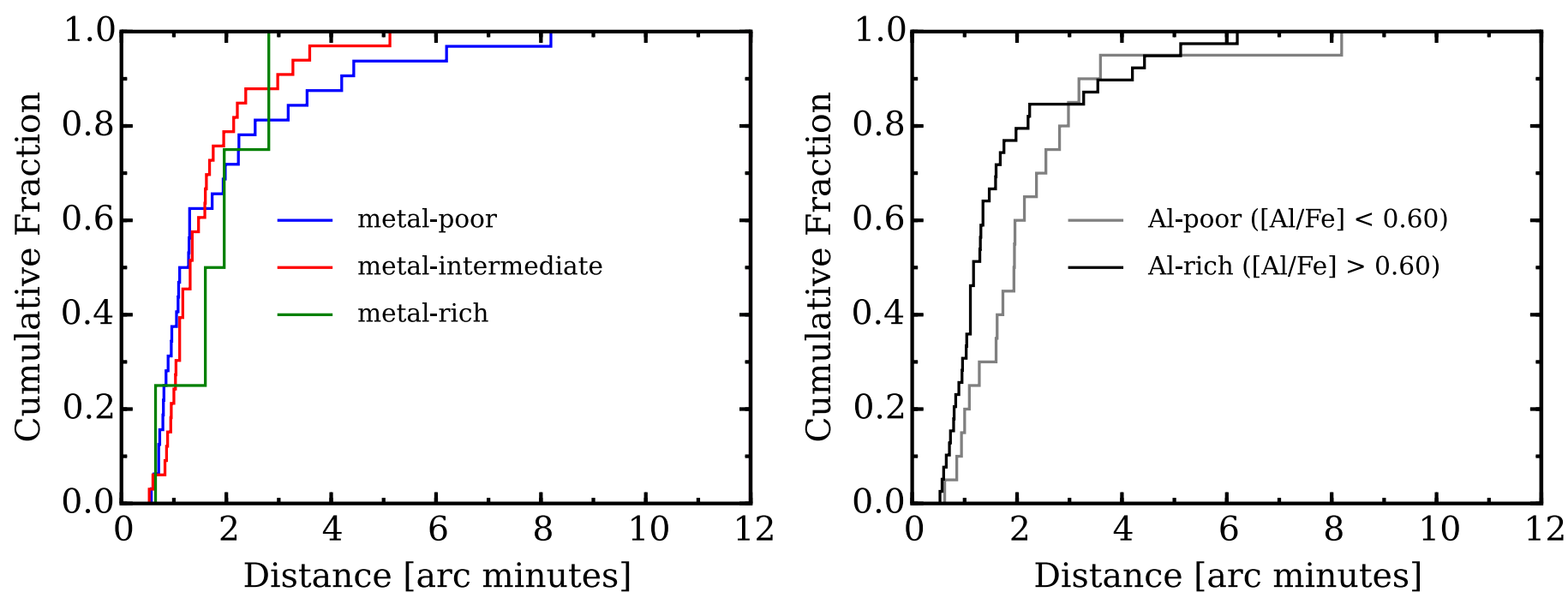

Figure 9. Left: the cumulative distribution functions of the metal-poor (blue), metal-intermediate (red), and metal-rich (green) populations are shown as a function of the projected distance from the cluster center. Note the preferential central concentration of metal-intermediate, and possibly metal-rich, stars at distances $\gtrsim 1$. 5 . Right: a similar plot comparing the radial distributions of Al-poor (gray; "first generation") and Al-rich (black; "second generation") stars from all three major populations. Note the significant central concentration of Al-rich stars.

inside $3^{\prime}$ of the cluster center (see also Figure 3), the sample size is too small to draw any strong conclusions about this population's radial distribution. For the two dominate populations, a difference in their radial distributions is only observed at projected distances $\gtrsim 1.5$ from the cluster center, and a twosided Kolmogorov-Smirnov test indicates that we do not have enough evidence to reject the null hypothesis that the two data sets are drawn from the same radial distribution. However, we note that the radial range where the distributions may differ is within $\sim 1-3$ half-mass radii, which is the region that Vesperini et al. (2013) estimate the local population mixtures may closely match the global ratios. Interestingly, if the radial segregation of stars with different metallicities is confirmed from larger sample sizes, then NGC 6273 would share a similar metallicity gradient morphology with $\omega$ Cen (e.g., Norris et al. 1996; Suntzeff \& Kraft 1996; Rey et al. 2004; Bellini et al. 2009; Johnson \& Pilachowski 2010). Such a gradient would contrast with NGC 1851, where Carretta et al. (2010b) found the metalpoor stars to be the most centrally concentrated.

\subsection{Additional Evidence of a Complex Metallicity Distribution}

Spectroscopic observations have indicated that several clusters, including $\omega$ Cen (e.g., Norris \& Da Costa 1995; Johnson \& Pilachowski 2010; Marino et al. 2011a), NGC 5286 (Marino et al. 2015), M2 (Yong et al. 2014), M54 (Carretta et al. 2010a), Terzan 5 (Origlia et al. 2013; Massari et al. 2014), NGC 1851 (Yong \& Grundahl 2008; Carretta et al. 2011; Lim et al. 2015), 
and M22 (Pilachowski et al. 1982; Da Costa et al. 2009; Marino et al. 2009, 2011b), may host multiple populations with distinct $[\mathrm{Fe} / \mathrm{H}]$ ratios. However, recent studies by Mucciarelli et al. (2015b) and Lardo et al. (2016) claim that at least some of these $[\mathrm{Fe} / \mathrm{H}]$ spreads are spurious detections driven by a disparity between $[\mathrm{Fe} \mathrm{I} / \mathrm{H}]$ and $[\mathrm{Fe} \mathrm{II} / \mathrm{H}]$. Similarly, Ivans et al. (2001), Lapenna et al. (2014), and Mucciarelli et al. (2015c) found that $[\mathrm{Fe} / \mathrm{H}]$ determinations for RGB and AGB stars can differ systematically by $>0.1$ dex, and that mixing RGB and AGB stars in a sample can produce an artificial metallicity spread. A common thread connecting these issues is the method by which a star's surface gravity is determined (spectroscopic versus photometric). Specifically, spectroscopic determinations may produce optimal $\log (g)$ values that correspond to masses that are systematically too low $\left(<0.5 M_{\odot}\right.$ in many cases). Since we utilize a spectroscopic surface gravity method and find that NGC 6273 shares many chemical and morphological characteristics with clusters such as M2 and M22, for which intrinsic $[\mathrm{Fe} / \mathrm{H}]$ spreads are contested, it is prudent to examine alternative lines of evidence that may support or refute NGC 6273 possessing an intrinsic metallicity spread.

Both spectroscopy and photometry unambiguously agree that $\omega$ Cen possesses discrete RGB populations with different $[\mathrm{Fe} / \mathrm{H}]$, and in Figure 10 we directly compare the spectra of NGC 6273 and $\omega$ Cen stars that have physical parameters typical of those in the metal-poor, metal-intermediate, and metal-rich groups. The $\omega$ Cen temperature and gravity parameters were determined entirely from photometric methods (see Johnson \& Pilachowski 2010), assuming masses of 0.8 $M_{\odot}$, and therefore should avoid the potential spectroscopic gravity problems noted above. As can be seen in Figure 10, the nearly identical Fe I and Fe II line profiles suggest that the NGC 6273 and $\omega$ Cen stars share similar compositions across a wide $[\mathrm{Fe} / \mathrm{H}]$ range. We note also that the $\mathrm{CaT}[\mathrm{Fe} / \mathrm{H}]$ distribution shown in Figure 8 for NGC 6273 closely matches the extended metallicity distribution found in $\omega$ Cen (e.g., Norris et al. 1996; Suntzeff \& Kraft 1996).

As shown in Figure 2, the broad color dispersion along the upper RGB provides some evidence that NGC 6273 may harbor an intrinsic metallicity spread. We investigate this further in Figure 11 by examining the upper RGB regions of the $(\mathrm{F} 336 \mathrm{~W})_{\mathrm{o}}$ versus $(\mathrm{F} 336 \mathrm{~W}-\mathrm{F} 555 \mathrm{~W})_{\mathrm{o}}$ and $(\mathrm{F} 555 \mathrm{~W})_{\mathrm{o}}$ versus (F438W-F555W)o color-magnitude diagrams and identifying the Bulge_GC1 spectroscopic targets with different $[\mathrm{Fe} / \mathrm{H}]$. Both color-magnitude diagrams indicate that the two dominant metallicity groups tend to separate on the upper RGB. The $(\mathrm{F} 336 \mathrm{~W})_{\mathrm{o}}$ versus $(\mathrm{F} 336 \mathrm{~W}-\mathrm{F} 555 \mathrm{~W})_{\mathrm{o}}$ plot in particular suggests that the brightest $\sim 0.5$ mag of the RGB-tip may split into at least two sequences with different $[\mathrm{Fe} / \mathrm{H}]$, which is similar to the result found by Marino et al. (2015) for NGC 5286. However, the F336W and F438W filters, and by extension the F336W-F555W and F438W-F555W colors, can be sensitive to both a star's overall metallicity and its $\mathrm{C}+\mathrm{N}+\mathrm{O}$ abundances. Therefore, the color-magnitude diagrams shown in Figure 11 are consistent with an intrinsic metallicity spread, but a detailed examination of the cluster's CNO (and also $\mathrm{He}$ ) abundances is required in order to fully confirm this result. Interestingly, the two metal-rich stars in Figure 11 are located at colors that are bluer than might be expected from their metallicities alone. We note that similar observations have been found for the equivalent "s-poor/Fe-rich" stars in NGC 5286 (Marino et al. 2015) and M2 (Yong et al. 2014). The bluer colors for these stars may reflect lower atmospheric opacities driven by different light element compositions and perhaps lower $[\alpha / \mathrm{Fe}]$ ratios, at least for NGC $6273 .^{23}$

Figure 11 also shows that very few of our Bulge_GC1 targets are on the AGB, indicating that the measured metallicity spread is not caused by systematic differences in the RGB and AGB abundance scales. In fact, the few AGB stars in our sample appear to belong to both the metal-poor and metalintermediate populations, and the two metal-rich stars could also belong to a more metal-rich AGB sequence. Therefore, we regard the combined evidence of separate sub-giant and RGB sequences observed by Han et al. (2015) with the $h k$ filter, the RGB color dispersions seen in Figures 2 and 11 here, the large metallicity spreads detected previously by Johnson et al. (2015b) and Yong et al. (2016), and the spectroscopic data presented here as strong evidence that NGC 6273 possesses an intrinsic metallicity spread.

\subsection{Light and Heavy Element Chemical Abundance Patterns}

\subsubsection{Alpha Element Abundances}

The $\alpha$ elements $\mathrm{Mg}, \mathrm{Si}$, and $\mathrm{Ca}$ are largely produced during hydrostatic and explosive carbon, neon, and oxygen burning in massive stars (e.g., Woosley \& Weaver 1995). In environments where chemical enrichment has been dominated by the products of core-collapse supernovae ( $\mathrm{SNe}$ ), one tends to find stars with $[\alpha / \mathrm{Fe}]$ abundances that are enhanced by about a factor of two to three over the solar ratio (e.g., see the review by McWilliam 1997). In contrast, longer enrichment timescales may produce stars with lower $[\alpha / \mathrm{Fe}]$ ratios as $\mathrm{SNe}$ Ia begin to contribute larger amounts of Fe-peak elements than $\alpha$ elements (Tinsley 1979).

As can be clearly seen in Gratton et al. (2004; their Figure 4), nearly all Galactic globular clusters have $[\alpha / \mathrm{Fe}] \sim$ $0.2-0.4$ dex. Furthermore, the star-to-star scatter of $[\alpha / \mathrm{Fe}]$ within a given cluster is typically $<0.1 \mathrm{dex}$, which suggests that the products of core-collapse SNe were well-mixed. Only a small number of clusters, such as Ruprecht 106, Terzan 7, and Palomar 12 , are known to have abnormally low $[\alpha / \mathrm{Fe}]$ ratios (e.g., Pritzl et al. 2005), and all three of these clusters are thought to have extragalactic/accretion origins (e.g., Cohen 2004; Law \& Majewski 2010; Villanova et al. 2013). Therefore, one does not normally expect to find stars with enhanced and depleted $[\alpha / \mathrm{Fe}]$ ratios within a single globular cluster, beyond the well-known proton-capture nucleosynthesis variations (see Section 5.3.2). In fact, only the massive ironcomplex clusters $\omega$ Cen, NGC 6273, M54, M2, and Terzan 5 show any evidence of hosting stars with different $[\alpha / \mathrm{Fe}]$ ratios (Pancino et al. 2002; Origlia et al. 2003, 2011, 2013; Carretta et al. 2010a; Johnson \& Pilachowski 2010; Yong et al. 2014; Johnson et al. 2015b).

Figure 12 and Table 8 show the $[\mathrm{Mg} / \mathrm{Fe}],[\mathrm{Si} / \mathrm{Fe}],[\mathrm{Ca} / \mathrm{Fe}]$, and averaged $[\alpha / \mathrm{Fe}]$ patterns of NGC 6273's various populations. In agreement with Johnson et al. (2015b), we find that most stars in NGC 6273 have elevated $\alpha$ element abundances, but that the average $[\mathrm{Mg} / \mathrm{Fe}]$ and $[\mathrm{Si} / \mathrm{Fe}]$ ratios may decrease slightly as a function of increasing metallicity. The $[\mathrm{Si} / \mathrm{Fe}]$ abundances in particular may show additional

\footnotetext{
23 We note that CNO variations are likely present in NGC 6273 since Han et al. (2015) found the more Ca-rich (metal-rich) stars to have enhanced $\mathrm{CN}$ and $\mathrm{CH}$. Additionally, a few of the most metal-rich stars in our data set have very strong $\mathrm{CN}$ lines.
} 

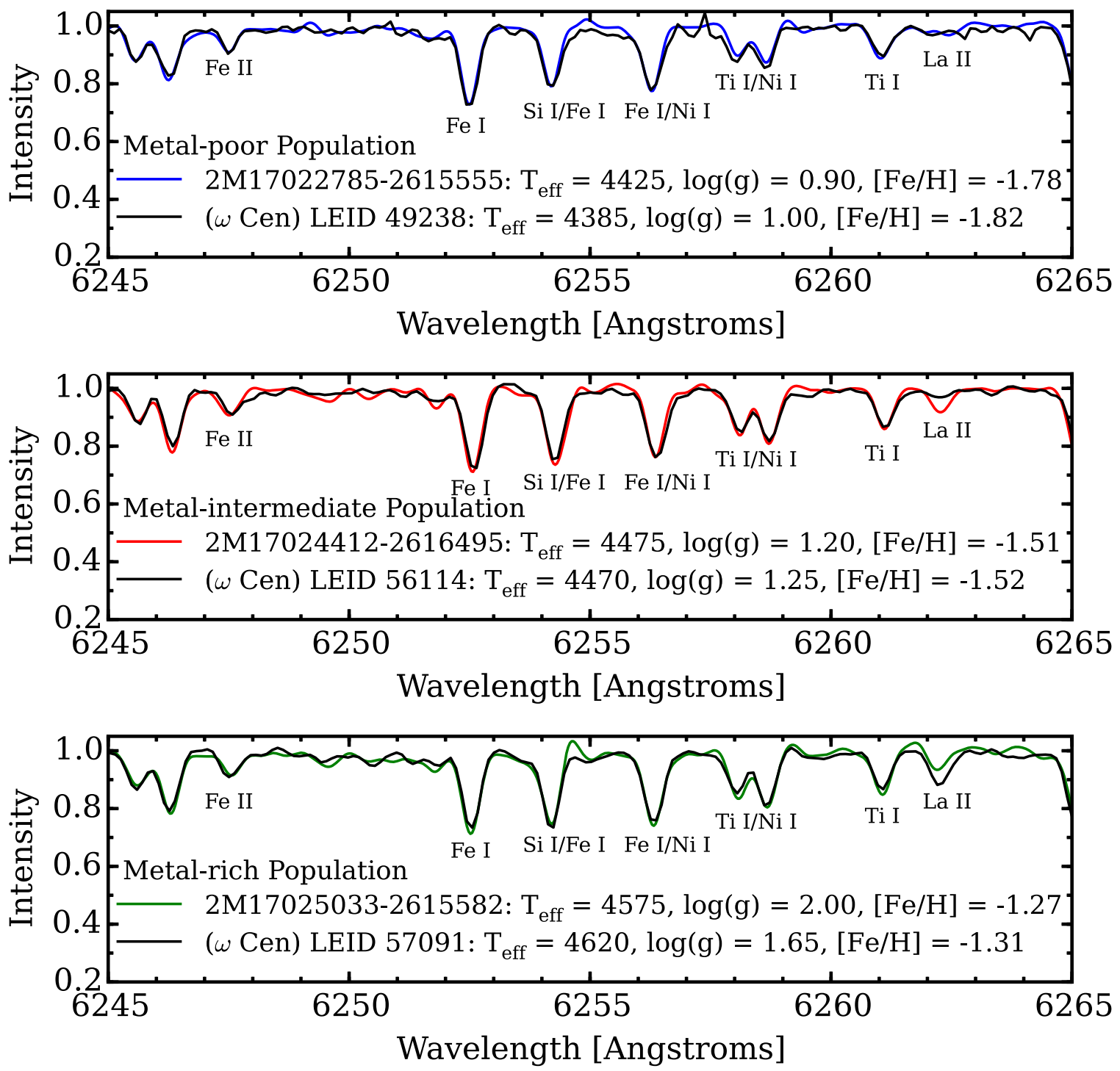

Figure 10. This figure compares the spectra of stars in NGC 6273 and $\omega$ Cen (Johnson \& Pilachowski 2010) that have similar $T_{\text {eff }}, \log (g)$, and [Fe/H]. The top, middle, and bottom panels show stars from the metal-poor, metal-intermediate, and metal-rich groups, respectively. In these panels, the colored spectra are from stars in NGC 6273 and the black spectra are from stars in $\omega$ Cen. The NGC 6273 M2FS spectra have been smoothed to match the resolution of the $\omega$ Cen Hydra spectra $(R \sim 18,000)$.

substructure, and we find some evidence that the average $[\mathrm{Si} / \mathrm{Fe}]$ abundances of the " $\alpha$-enhanced" metal-intermediate stars may be lower than those of the metal-poor and metal-rich groups. We note that a similar change in the $[\mathrm{Si} / \mathrm{Fe}]$ abundances with $[\mathrm{Fe} / \mathrm{H}]$ has been observed in $\omega$ Cen (Johnson \& Pilachowski 2010), which suggests that this trend could be the signature of a particular self-enrichment mode in massive clusters. However, the $[\mathrm{Ca} / \mathrm{Fe}]$ abundances show no significant trends as a function of $[\mathrm{Fe} / \mathrm{H}]$, and the typical dispersion within each sub-population is $\sim 0.1$ dex.

In a previous analysis, Johnson et al. (2015b) discovered that the most metal-rich star in their sample exhibited low $[\mathrm{X} / \mathrm{Fe}]$ ratios for several species, including the $\alpha$ elements. The new data presented here indicate that not all metal-rich stars have low $[\alpha / \mathrm{Fe}]$, but we find at least five "low- $\alpha$ " stars that have approximately solar $[\mathrm{Mg} / \mathrm{Fe}],[\mathrm{Si} / \mathrm{Fe}]$, and $[\mathrm{Ca} / \mathrm{Fe}]$ abundances. As can be seen in Figure 12, all five low- $\alpha$ stars have $[\mathrm{Fe} / \mathrm{H}]>-1.5$ dex. Additionally, the specific frequency of low- $\alpha$ stars increases with metallicity such that these stars constitute $9 \%(3 / 33)$ of the metal-intermediate population and
$50 \%(2 / 4)$ of the metal-rich population. However, we caution that the measured specific frequency values are likely affected by small number statistics and should be confirmed with additional observations.

Although we noted above that $\omega$ Cen, M54, M2, and Terzan 5 also contain stars with higher $[\mathrm{Fe} / \mathrm{H}]$ and lower $[\alpha / \mathrm{Fe}]$, none of these clusters exactly matches the pattern of NGC 6273 . For example, the $\alpha$-poor stars in M2 and Terzan 5 are exclusively found in the most metal-rich populations, but neither cluster appears to contain $\alpha$-enhanced and $\alpha$-poor stars at the same metallicity. Although Johnson \& Pilachowski (2010; see their Figure 10) found several stars with high and low $[\mathrm{Si} / \mathrm{Fe}]$ and $[\mathrm{Ca} / \mathrm{Fe}]$ abundances across a broad range of $[\mathrm{Fe} / \mathrm{H}]$ in $\omega \mathrm{Cen}$, follow-up observations are required to confirm that this pattern matches what is found in NGC 6273.

Interestingly, the M54 cluster and Sagittarius nuclear field star system may provide the closest example to what is observed in NGC 6273 (see Carretta et al. 2010a). In this system, the metal-poor cluster M54 contains a metallicity spread but only $\alpha$-enhanced stars. In contrast, the surrounding 

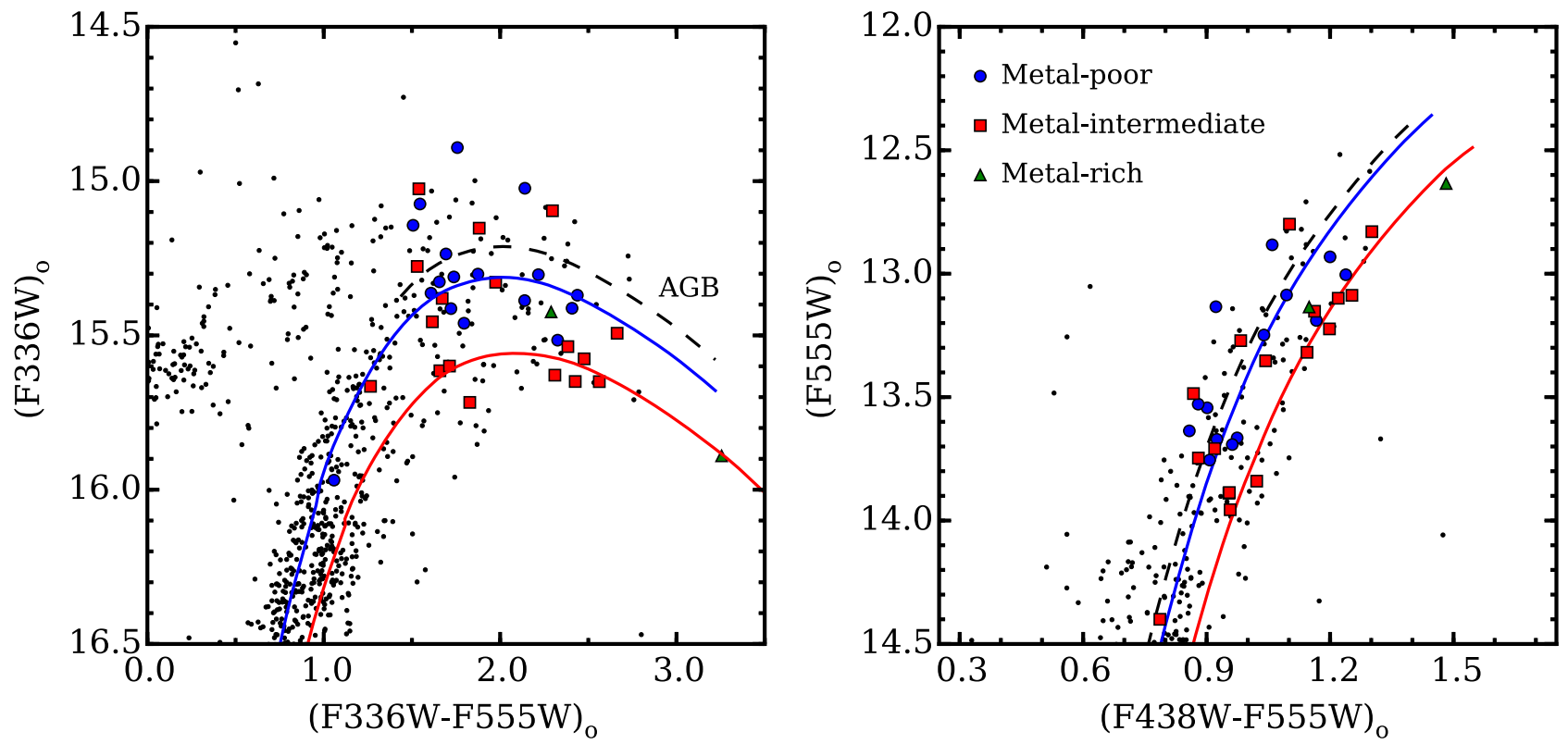

Figure 11. Left and right panels compare the upper RGB and AGB regions of NGC 6273 using combinations of the dereddened F336W, F438W, and F555W bands. The Bulge_GC1 observations that overlap with the WFC3 field of view are distinguished by metallicity using the same criteria, colors, and symbols as those in Figure 7. The two panels include Dartmouth isochrones with ages of $12 \mathrm{Gyr},[\alpha / \mathrm{Fe}]=0.4$ dex, distances of $9 \mathrm{kpc}$, and $[\mathrm{Fe} / \mathrm{H}]=-1.75$ (blue lines) and -1.50 (red lines) dex, which correspond to the metallicities of the two dominant populations. The dashed black lines separate the RGB and AGB stars. The bluer colors of the most metal-rich stars (green triangles) suggest that these stars may have different $\mathrm{He}, \mathrm{C}, \mathrm{N}$, and $\mathrm{O}$ abundances than the metal-intermediate population and/or may be higher metallicity AGB stars.

galaxy field stars are generally more metal-rich and have lower $[\alpha / \mathrm{Fe}]$. Therefore, if NGC 6273 formed in the core of a system similar to the Sagittarius dwarf galaxy, then the cluster may have been able to accrete a small number of metal-rich, $\alpha$-poor field stars from its progenitor population. Alternatively, the low- $\alpha$ stars in NGC 6273 may have been preferentially polluted by the ejecta of SNe Ia, perhaps in a scenario similar to that discussed in D'Antona et al. (2016). However, such a scenario would have to be able to produce low- $\alpha$ stars with different $[\mathrm{Fe} / \mathrm{H}]$ but otherwise similar compositions (see Sections 5.3.2 and 5.3.3), and may even have to occur multiple times in clusters like NGC 6273.

\subsubsection{Light Element Abundances}

As mentioned in Section 1, globular clusters show clear light element abundance variations that extend beyond the effects of first dredge-up and are a result of high temperature (>40 MK; Langer et al. 1993, 1997; Prantzos et al. 2007) proton-capture burning. Since these effects are observed in main-sequence and evolved RGB stars (e.g., Gratton et al. 2001), we know that the gas from which present day cluster stars formed was polluted by a previous generation of more massive stars. Although the exact nucleosynthesis sources remain a mystery, the observed effects include anti-correlations among the element pairs $\mathrm{C}-\mathrm{N}$, $\mathrm{O}-\mathrm{N}, \mathrm{O}-\mathrm{Na}, \mathrm{O}-\mathrm{Al}$, and $\mathrm{Mg}-\mathrm{Al}$ and correlations of $\mathrm{C}-\mathrm{O}$, $\mathrm{N}-\mathrm{Na}$, and $\mathrm{Na}-\mathrm{Al}$ (e.g., Sneden et al. 2004). He enhancements are also likely found in stars with low $\mathrm{O} / \mathrm{Mg}$ and high $\mathrm{Na} / \mathrm{Al}$ (e.g., Bragaglia et al. 2010a, 2010b; Dupree et al. 2011). For this paper, we adopt the common nomenclature that "first generation" stars are those with compositions similar to metalpoor halo field stars (i.e., lower $\mathrm{He}, \mathrm{N}, \mathrm{Na}$, and $\mathrm{Al}$ abundances; higher $\mathrm{C}, \mathrm{O}$, and $\mathrm{Mg}$ abundances) and "second generation" stars are those with enhanced $\mathrm{He}, \mathrm{N}, \mathrm{Na}$, and $\mathrm{Al}$ abundances and depleted $\mathrm{C}, \mathrm{O}$, and possibly $\mathrm{Mg}$ abundances.
The $\mathrm{Mg}-\mathrm{Al}$ anti-correlation is only found in a handful of the most massive clusters, but may be particularly useful for identifying discrete populations (e.g., Carretta 2014, 2015). Since the full $\mathrm{Mg}-\mathrm{Al}$ cycle is activated at a higher temperature than the $\mathrm{O}-\mathrm{N}$ and $\mathrm{Ne}-\mathrm{Na}$ cycles, the presence (or not) of a $\mathrm{Mg}-$ $\mathrm{Al}$ anti-correlation provides important insight into the burning temperatures achieved by the pollution sources. Similarly, a few of the most massive clusters also exhibit abundance variations that extend to elements as heavy as $\mathrm{Si}, \mathrm{K}$, and $\mathrm{Sc}$, which is likely a byproduct of even higher temperature protoncapture burning (Yong et al. 2005; Carretta et al. 2009b, 2013, 2014; Johnson \& Pilachowski 2010; Cohen \& Kirby 2012; Mucciarelli et al. 2012, 2015a; Ventura et al. 2012; Carretta 2015; Roederer \& Thompson 2015). Notably, many of these clusters share similar properties with NGC 6273, such as extended blue HBs.

In Figure 13 and Table 8, we compare the $[\mathrm{Na} / \mathrm{Fe}],[\mathrm{Mg} / \mathrm{Fe}]$, $[\mathrm{Al} / \mathrm{Fe}]$, and $[\alpha / \mathrm{Fe}]$ abundances of the three different metallicity groups in NGC 6273. Similar to the results of Johnson et al. (2015b), we find that both $[\mathrm{Na} / \mathrm{Fe}]$ and $[\mathrm{Al} / \mathrm{Fe}]$ vary by about factors of 5 and 10 , respectively, and that clear $\mathrm{Na}-\mathrm{Al}$ correlations are independently present in the metal-poor, metal-intermediate, and metal-rich populations. Therefore, NGC 6273 shares a common feature observed in other iron-complex clusters: each population with a unique metallicity was able to generate its own independent spread of light element abundances that closely resembles the patterns exhibited by monometallic clusters.

The $\mathrm{Na}-\mathrm{Al}$ correlation in Figure 13 shows a paucity of stars near $[\mathrm{Al} / \mathrm{Fe}] \sim 0.6 \mathrm{dex}$. If we adopt this cut-off as the discriminator between first and second generation stars, then we find that approximately two-thirds of the cluster stars can be classified as second generation stars. The metal-poor and metalintermediate populations each favor second generation stars with first:second generation ratios of 35\%:65\% and 28\%:72\%, 

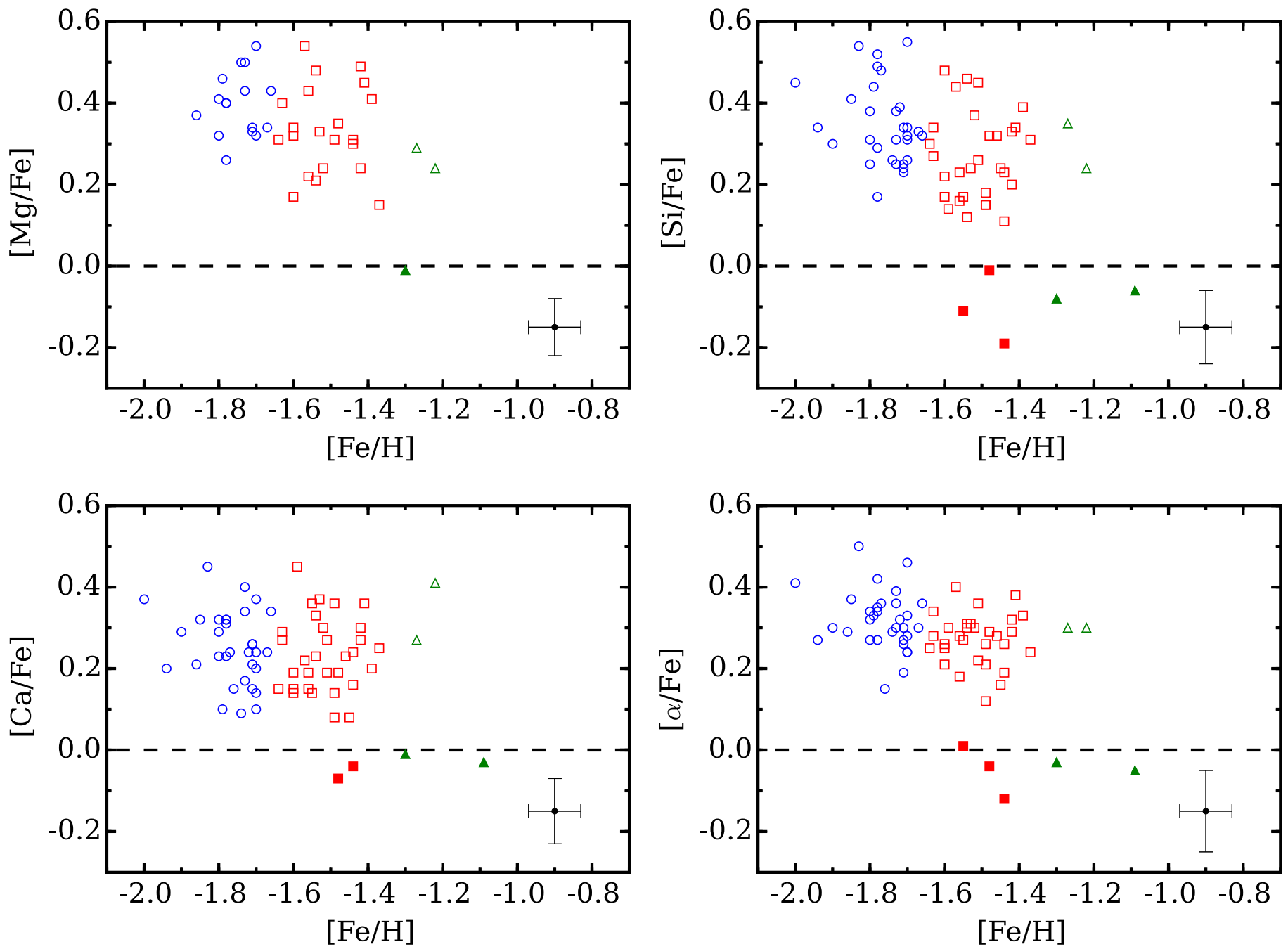

Figure 12. $[\mathrm{Mg} / \mathrm{Fe}],[\mathrm{Si} / \mathrm{Fe}],[\mathrm{Ca} / \mathrm{Fe}]$, and $[\alpha / \mathrm{Fe}]$ ratios for NGC 6273 stars observed in this work and Johnson et al. (2015b) are plotted as a function of [Fe/H]. The open blue circles, red boxes, and green triangles designate stars belonging to the metal-poor, metal-intermediate, and metal-rich populations, respectively. The filled symbols indicate stars that have low $[\mathrm{Mg} / \mathrm{Fe}],[\mathrm{Si} / \mathrm{Fe}],[\mathrm{Ca} / \mathrm{Fe}]$, and $[\alpha / \mathrm{Fe}]$ abundances. The dashed black lines indicate the solar $[\mathrm{X} / \mathrm{Fe}]$ ratios, and the $[\alpha / \mathrm{Fe}]$ abundances represent the average values of $[\mathrm{Mg} / \mathrm{Fe}],[\mathrm{Si} / \mathrm{Fe}]$, and $[\mathrm{Ca} / \mathrm{Fe}]$ measured in each star. Typical error bars are included in the bottom right corner of each plot.

Table 8

Mean Composition Properties

\begin{tabular}{|c|c|c|c|c|c|c|c|c|c|c|}
\hline Statistic & $\begin{array}{c}{[\mathrm{Fe} / \mathrm{H}]} \\
(\mathrm{dex})\end{array}$ & $\begin{array}{c}{[\mathrm{Na} / \mathrm{Fe}]} \\
(\mathrm{dex})\end{array}$ & $\begin{array}{c}{[\mathrm{Mg} / \mathrm{Fe}]} \\
(\mathrm{dex})\end{array}$ & $\begin{array}{c}{[\mathrm{Al} / \mathrm{Fe}]} \\
(\mathrm{dex})\end{array}$ & $\begin{array}{c}{[\mathrm{Si} / \mathrm{Fe}]} \\
(\mathrm{dex})\end{array}$ & $\begin{array}{c}{[\mathrm{Ca} / \mathrm{Fe}]} \\
(\mathrm{dex})\end{array}$ & $\begin{array}{c}{[\mathrm{Cr} / \mathrm{Fe}]} \\
(\mathrm{dex})\end{array}$ & $\begin{array}{c}{[\mathrm{Ni} / \mathrm{Fe}]} \\
(\mathrm{dex})\end{array}$ & $\begin{array}{c}{[\mathrm{La} / \mathrm{Fe}]} \\
(\mathrm{dex})\end{array}$ & $\begin{array}{c}{[\mathrm{Eu} / \mathrm{Fe}]} \\
(\mathrm{dex})\end{array}$ \\
\hline \multicolumn{11}{|c|}{ Metal-poor Population } \\
\hline Average & -1.77 & 0.30 & 0.40 & 0.74 & 0.35 & 0.25 & 0.00 & -0.03 & 0.15 & 0.39 \\
\hline$\sigma$ & 0.08 & 0.19 & 0.08 & 0.34 & 0.10 & 0.09 & 0.10 & 0.10 & 0.24 & 0.15 \\
\hline \multicolumn{11}{|c|}{ Metal-intermediate Population } \\
\hline Average & -1.51 & 0.26 & 0.34 & 0.75 & 0.23 & 0.22 & 0.02 & -0.06 & 0.47 & 0.36 \\
\hline$\sigma$ & 0.07 & 0.20 & 0.10 & 0.27 & 0.15 & 0.11 & 0.13 & 0.10 & 0.28 & 0.12 \\
\hline \multicolumn{11}{|c|}{ Metal-rich Population } \\
\hline Average & -1.22 & 0.19 & 0.17 & 0.46 & 0.11 & 0.16 & 0.02 & -0.11 & 0.57 & 0.42 \\
\hline$\sigma$ & 0.09 & 0.34 & 0.16 & 0.41 & 0.21 & 0.21 & 0.21 & 0.21 & 0.40 & 0.21 \\
\hline
\end{tabular}

respectively. On the other hand, the metal-rich population has a ratio of $75 \%: 25 \%$, but this measurement is based on only four stars. Therefore, the numerical dominance of second generation stars in NGC 6273 fits a common trend observed in many Galactic globular clusters (e.g., Carretta et al. 2009c, see their Figure 10). Similarly, Figure 9 shows that the second generation stars in NGC 6273 are more centrally concentrated than the first generation stars, which again matches a pattern 

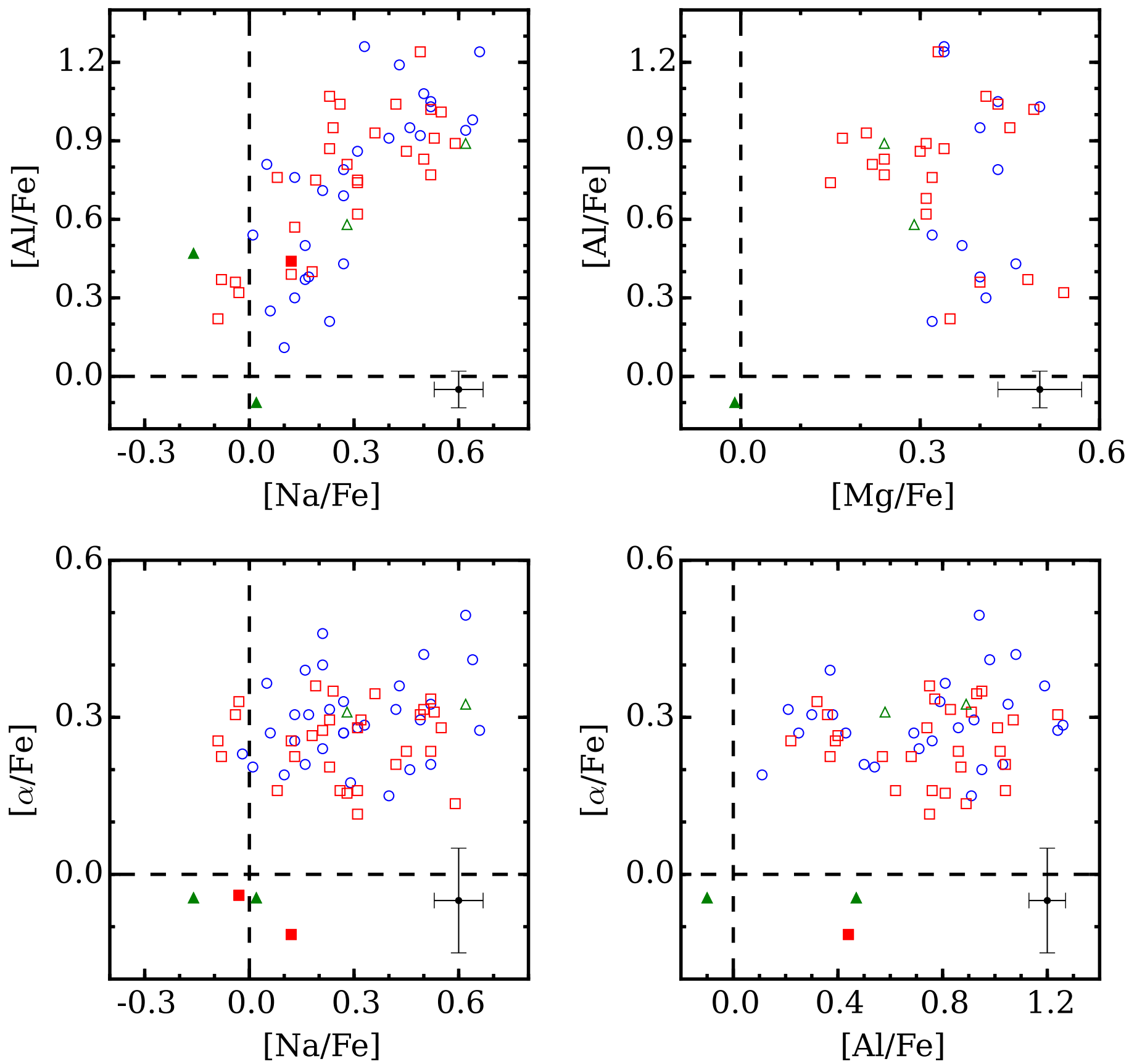

Figure 13. Top: these panels show the NGC 6273 [Al/Fe] abundances from this work and Johnson et al. (2015b) plotted as a function of [Na/Fe] (left) and [Mg/Fe] (right). A clear $\mathrm{Na}-\mathrm{Al}$ correlation exists for all three metallicity groups, but a $\mathrm{Mg}-\mathrm{Al}$ anti-correlation may only be present in the metal-intermediate and metal-rich populations. The colors and symbols are the same as those in Figure 12. Bottom: these panels plot the $[\alpha / \mathrm{Fe}]$ ratios as a function of $[\mathrm{Na} / \mathrm{Fe}]$ (left) and $[\mathrm{Al} / \mathrm{Fe}]$ (right). Note that all of the "low- $\alpha$ " stars have low $[\mathrm{Na} / \mathrm{Fe}]$ and $[\mathrm{Al} / \mathrm{Fe}]$ abundances.

observed in many iron-complex and monometallic clusters (e.g., Lardo et al. 2011). We note also that an additional paucity of stars may be present near $[\mathrm{Na} / \mathrm{Fe}] \sim 0.35 \mathrm{dex}$, which may further distinguish the most $\mathrm{Na} / \mathrm{Al}$-rich stars. These stars, which constitute $\sim 33 \%$ of our sample, are likely equivalent to the "extreme" population found by Carretta et al. (2009c) in several clusters, the "E" population of NGC 2808 (Milone et al. 2015b), and the faint sub-giant branch stars of 47 Tuc (Marino et al. 2016). We note that a similarly high fraction of very $\mathrm{Na} / \mathrm{Al}$-rich stars is also found in $\omega$ Cen (Johnson \& Pilachowski 2010; Marino et al. 2011a) and M54 (Carretta et al. 2010a).
For $[\mathrm{Mg} / \mathrm{Fe}]$ and $[\mathrm{Al} / \mathrm{Fe}]$, Figure 13 shows that the behavior of the element pair may change for stars of different metallicity in NGC 6273. The metal-poor component shows no correlation between $[\mathrm{Mg} / \mathrm{Fe}]$ and $[\mathrm{Al} / \mathrm{Fe}]$, but the metal-intermediate population shows evidence of a $\mathrm{Mg}-\mathrm{Al}$ anti-correlation for stars with $[\mathrm{Al} / \mathrm{Fe}]<1.0$ dex. A similar $\mathrm{Mg}-\mathrm{Al}$ anti-correlation may also be present for the metal-rich stars, but the sample size (four stars) is too small to draw any clear conclusions. Since ${ }^{24} \mathrm{Mg}$ is only significantly depleted at temperatures $\gtrsim 65 \mathrm{MK}$ (e.g., see Prantzos et al. 2007; their Figure 2), the different $\mathrm{Mg}-\mathrm{Al}$ relations for the metal-poor and metal-intermediate stars suggest that the gas from which each population's second 
generation stars formed was processed at different temperatures. However, we did not find any residual correlations between $\mathrm{Mg}$ or $\mathrm{Al}$ and the heavier elements like $\mathrm{Si}$, which indicates that the pollution source(s) responsible for the $\mathrm{Mg}-\mathrm{Al}$ anti-correlation in NGC 6273 likely did not reach temperatures high enough to significantly activate the ${ }^{27} \mathrm{Al}(\mathrm{p}, \gamma){ }^{28} \mathrm{Si}$ reaction related to the $\mathrm{Mg}-\mathrm{Al}$ chain.

An examination of $\mathrm{Mg}-\mathrm{Al}$ trends in the iron-complex clusters $\omega$ Cen, M54, M2, M22, NGC 1851, and NGC 5286 revealed that only $\omega$ Cen (Norris \& Da Costa 1995; Smith et al. 2000; Da Costa et al. 2013), M54 (Carretta et al. 2010a), and NGC 1851 (Carretta et al. 2011, 2012) exhibit evidence of $\mathrm{Mg}-\mathrm{Al}$ anti-correlations. Unlike NGC 6273, none of these clusters show evidence that the presence of a $\mathrm{Mg}-\mathrm{Al}$ anti-correlation depends on a population's metallicity. However, we note that in $\omega$ Cen the metalintermediate and metal-rich stars exhibit clear changes in their light element patterns (e.g., Norris \& Da Costa 1995; Johnson \& Pilachowski 2010; Marino et al. 2011a). For example, the very O-poor/Na-rich stars that dominate by number at higher metallicity are not found at $[\mathrm{Fe} / \mathrm{H}] \lesssim-1.8$, and $\mathrm{O}$ and $\mathrm{Na}$ are actually correlated in the most metal-rich stars. Additionally, for M54, Carretta et al. (2010a) found that the light element variations are more extended for the metal-rich stars than the metal-poor population. Therefore, NGC 6273, $\omega$ Cen, and M54 provide evidence that a cluster's enrichment signature can change with time, and that multiple pollution sources may be able to produce chemical patterns that are similar for some element pairs (e.g., $\mathrm{Na}-\mathrm{Al}$ ) but not others (e.g., $\mathrm{Mg}-\mathrm{Al}$ ).

Interestingly, Figure 13 shows that the metal-intermediate stars with $[\mathrm{Al} / \mathrm{Fe}]>1.0$ dex have $[\mathrm{Mg} / \mathrm{Fe}] \sim 0.4$ dex, rather than the $[\mathrm{Mg} / \mathrm{Fe}] \sim 0.0$ dex abundances that might be expected. The reason for this discrepancy is not immediately clear, but we note that many similar metallicity clusters have stars with $[\mathrm{Mg} / \mathrm{Fe}] \sim$ 0.4 dex and $[\mathrm{Al} / \mathrm{Fe}] \sim 1.0$ dex (e.g., Carretta et al. 2010b; see their Figure 6). Additionally, we note that Norris \& Da Costa (1995) found that intermediate metallicity stars in $\omega$ Cen could have $[\mathrm{Al} / \mathrm{Fe}]>1.0$ dex but $[\mathrm{Mg} / \mathrm{Fe}]$ could range from about $0.6 \mathrm{dex}$ (no $\mathrm{Mg}-\mathrm{Al}$ anti-correlation) to $0.0 \mathrm{dex}$ (clear $\mathrm{Mg}-\mathrm{Al}$ anticorrelation; see also Carretta et al. 2010a, their Figure 18). In this context, an examination of the ${ }^{24} \mathrm{Mg},{ }^{25} \mathrm{Mg}$, and ${ }^{26} \mathrm{Mg}$ abundances in NGC 6273, similar to the analysis of Da Costa et al. (2013) in $\omega$ Cen, could be particularly illuminating. However, we also caution that the $6319 \AA \mathrm{Mg}$ I lines used here are relatively weak, especially in stars with intrinsically low $[\mathrm{Mg} / \mathrm{Fe}]$, so the exact shape of the $\mathrm{Mg}-\mathrm{Al}$ anti-correlation should be confirmed with additional analyses.

Finally, we note that all of the low $\alpha$ stars have $[\mathrm{Na} / \mathrm{Fe}]$ and $[\mathrm{Al} / \mathrm{Fe}]$ compositions that are consistent with those of first generation stars. Although the sample size of low- $\alpha$ stars is small, a simulation of $10^{5}$ random draws from our $\alpha$-enhanced population indicated that there is only about a $0.05 \%$ chance that we would randomly draw five stars that have $[\mathrm{Na} / \mathrm{Fe}]<0.25 \mathrm{dex}$ and $[\mathrm{Al} / \mathrm{Fe}]<0.50$ dex. Therefore, we speculate that the low $-\alpha$ population may have been unable to form second generation stars. The pattern of low $[\mathrm{Na} / \mathrm{Fe}]$ and $[\mathrm{Al} / \mathrm{Fe}]$ abundances in the low- $\alpha$ stars of NGC 6273 mirrors the composition differences found by Carretta et al. (2010a) when comparing the M54 cluster and Sagittarius galaxy field stars. The similar composition patterns of the low- $\alpha$ stars in NGC 6273 and the Sagittarius field stars strengthens the idea that NGC 6273 may have accreted its low- $\alpha$ stars from a surrounding field population that was once part of a now dispersed dwarf galaxy.

\subsubsection{RGB Versus AGB Abundance Patterns}

As noted by Gratton et al. (2010) and many previous authors (e.g., Mallia 1978; Norris et al. 1981; Suntzeff 1981; Smith \& Norris 1993; Pilachowski et al. 1996b; Ivans et al. 1999; Sneden et al. 2000), some globular clusters may contain RGB and AGB populations with different light element abundances. Specifically, RGB stars that evolve onto the HB with masses $\lesssim 0.55 M_{\odot}$, presumably those with the highest $\mathrm{He}, \mathrm{N}, \mathrm{Na}$, and $\mathrm{Al}$ abundances and lowest $\mathrm{C}, \mathrm{O}$, and $\mathrm{Mg}$ abundances, may not ascend the AGB and instead end their lives as AGB-manqué stars (e.g., Greggio \& Renzini 1990). As a result, we expect to find that the light element abundance distributions of AGB stars should exhibit a paucity of second generation stars when compared to the RGB ratios.

Renewed interest in this field has produced somewhat conflicting results with the missing second generation fraction ranging from 100\% (Campbell et al. 2013; MacLean et al. 2016) to only a few percent (Johnson \& Pilachowski 2012; García-Hernández et al. 2015; Johnson et al. 2015a; Lapenna et al. 2016; Wang et al. 2016). However, the growing consensus is that only the most extreme second generation stars probably fail to ascend the AGB.

Since Figure 14 shows that NGC 6273 contains a very extended blue $\mathrm{HB}$, and that $\sim 30 \%$ of the cluster's HB stars have masses $\lesssim 0.55 M_{\odot}$, we investigate here whether any second generation stars may have failed to ascend the AGB. We restrict the comparison to only the targets shown in Figure 11 since these are the only stars in our sample that can be reliably assigned to either the RGB or AGB sequences. Although the sample sizes are small (9 AGB; 28 RGB), we find similar first:second generation ratios of $24 \%: 76 \%$ and 14\%:86\% for the RGB and AGB samples, respectively. However, further inspection of the $[\mathrm{Na} / \mathrm{Fe}]$ and $[\mathrm{Al} / \mathrm{Fe}]$ distributions in Figure 15 reveals that the AGB sample does not contain stars with $[\mathrm{Na} / \mathrm{Fe}]>0.5 \mathrm{dex}$ nor $[\mathrm{Al} / \mathrm{Fe}]>$ 1.0 dex. In other words, only the most $\mathrm{Na} / \mathrm{Al}-\mathrm{rich}$, and presumably He-enhanced, stars may have failed to ascend the AGB.

It is possible that the paucity of extreme Na/Al-rich AGB stars is a product of our small sample size. To investigate this, we performed $10^{5}$ random draws of an equivalent AGB sample from the RGB distribution, and we found about a $7 \%$ chance that the missing Na/Al-rich AGB stars could be due to the small sample size. Interestingly, the missing $\mathrm{Na} / \mathrm{Al}$-rich AGB stars account for $\sim 30 \%$ of the RGB sample, which is comparable to the fraction of extreme $\mathrm{HB}$ and blue hook stars found on the HB (see Figure 14 and Section 5.4). Therefore, we conclude that the NGC 6273 RGB stars with $[\mathrm{Na} / \mathrm{Fe}]>0.5$ dex and $[\mathrm{Al} / \mathrm{Fe}]>1.0$ dex likely evolve to become extreme $\mathrm{HB}$ or blue hook stars and fail to ascend the AGB.

\subsubsection{Fe-peak Element Abundances}

The Fe-peak elements $\mathrm{Cr}$ and $\mathrm{Ni}$ are largely produced in the late burning stages of massive stars, but include some production by $\mathrm{SNe}$ Ia as well (e.g., Timmes et al. 1995). Within a single globular cluster, the star-to-star scatter in $[\mathrm{Ni} / \mathrm{Fe}]$ and $[\mathrm{Cr} / \mathrm{Fe}]$ is typically $\lesssim 0.1$ dex (e.g., Gratton et al. 2004, see their Figure 2). Similarly, the average $[\mathrm{Cr} / \mathrm{Fe}]$ and $[\mathrm{Ni} / \mathrm{Fe}]$ ratios are about solar 

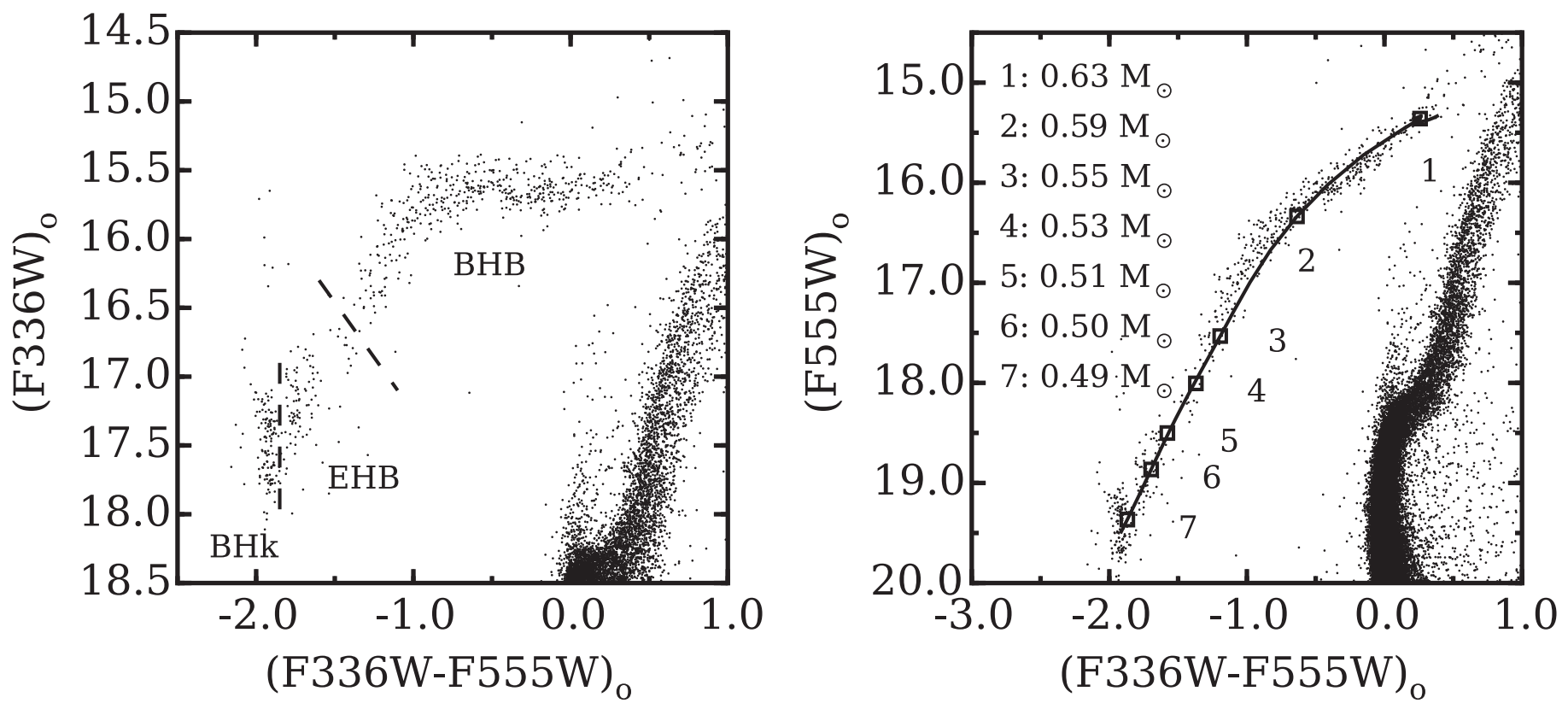

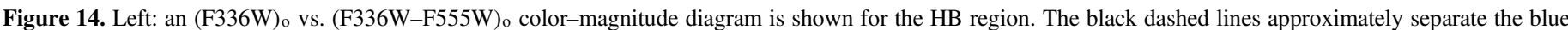

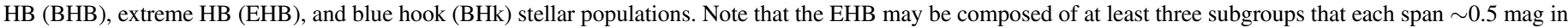

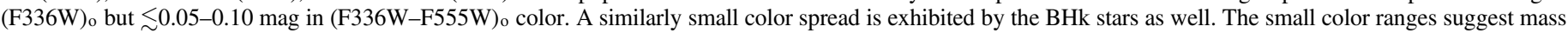

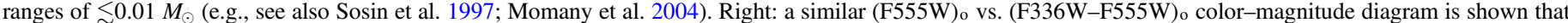

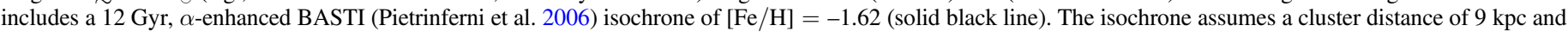

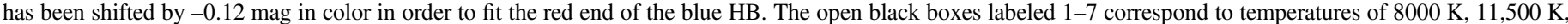
$16,675 \mathrm{~K}, 20,000 \mathrm{~K}, 22,500 \mathrm{~K}, 25,800 \mathrm{~K}$, and $32,000 \mathrm{~K}$, respectively.
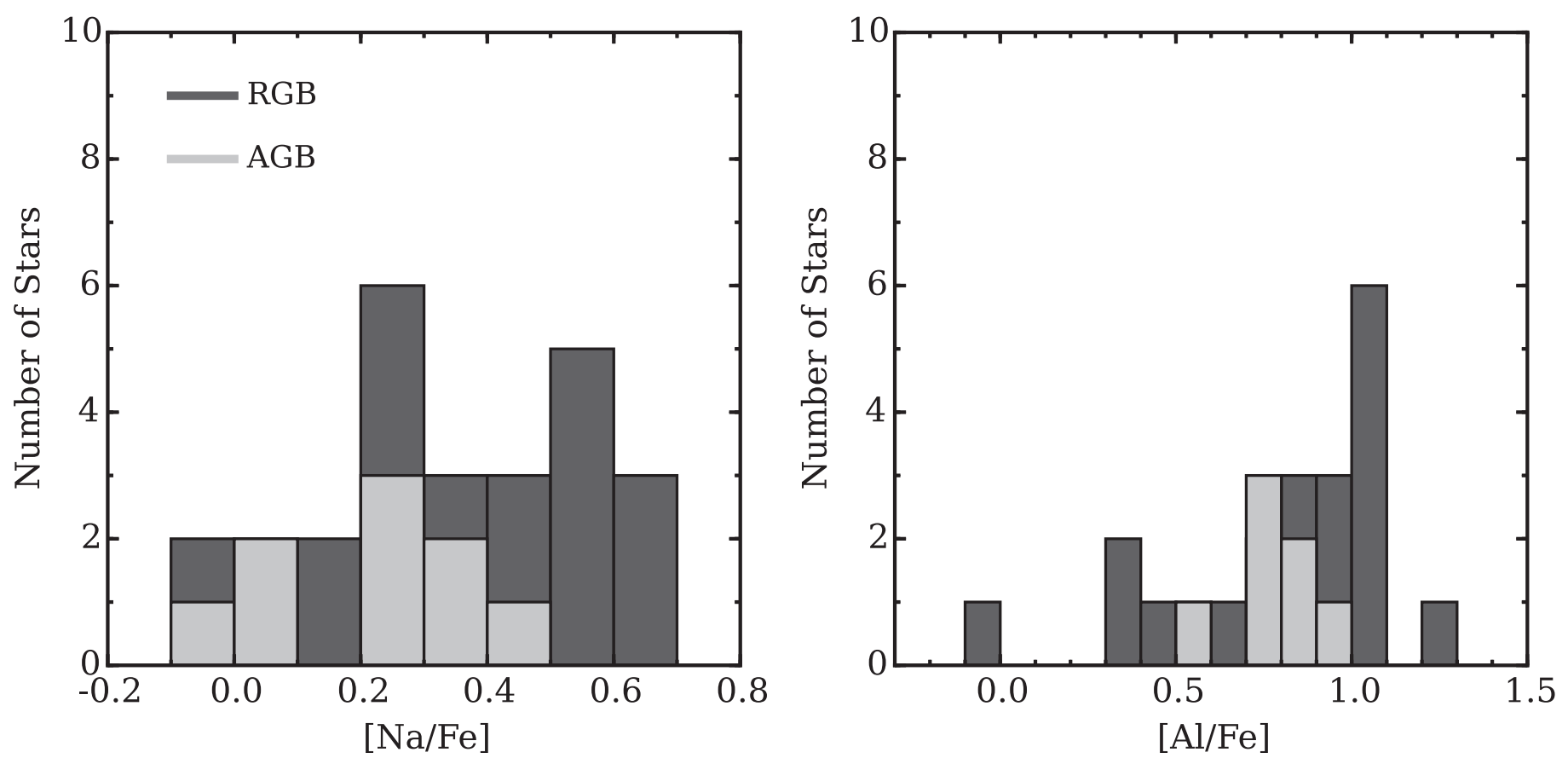

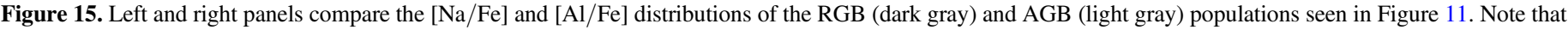
the AGB stars span a smaller range in both $[\mathrm{Na} / \mathrm{Fe}]$ and $[\mathrm{Al} / \mathrm{Fe}]$, and that no $\mathrm{AGB}$ stars were observed to have $[\mathrm{Na} / \mathrm{Fe}]>0.50 \mathrm{dex}$ and $[\mathrm{Al} / \mathrm{Fe}]>1.0 \mathrm{dex}$.

across the entire metallicity range spanned by clusters in the Galaxy.

In Figure 16 and Table 8, we show the abundance patterns of $[\mathrm{Cr} / \mathrm{Fe}]$ and $[\mathrm{Ni} / \mathrm{Fe}]$ for NGC 6273. Overall, we find $\langle[\mathrm{Cr} / \mathrm{Fe}]\rangle=0.01 \mathrm{dex}(\sigma=0.12 \mathrm{dex})$ and $\langle[\mathrm{Ni} / \mathrm{Fe}]\rangle=$ $-0.05 \operatorname{dex}(\sigma=0.11 \mathrm{dex})$, which is in agreement with Johnson et al. (2015b). An examination of Figure 16 shows that the metal-poor, metal-intermediate, and metal-rich stars all exhibit nearly identical $[\mathrm{Cr} / \mathrm{Fe}]$ and $[\mathrm{Ni} / \mathrm{Fe}]$ abundances and dispersions. However, we note that several (but not all) of the low- $\alpha$ stars have $[\mathrm{Cr}, \mathrm{Ni} / \mathrm{Fe}] \lesssim-0.2 \mathrm{dex}$, similar to what is found in some clusters associated with the Sagittarius dwarf galaxy. A detailed examination of key Fe-peak elements that are sensitive to nucleosynthesis processes operating in different 

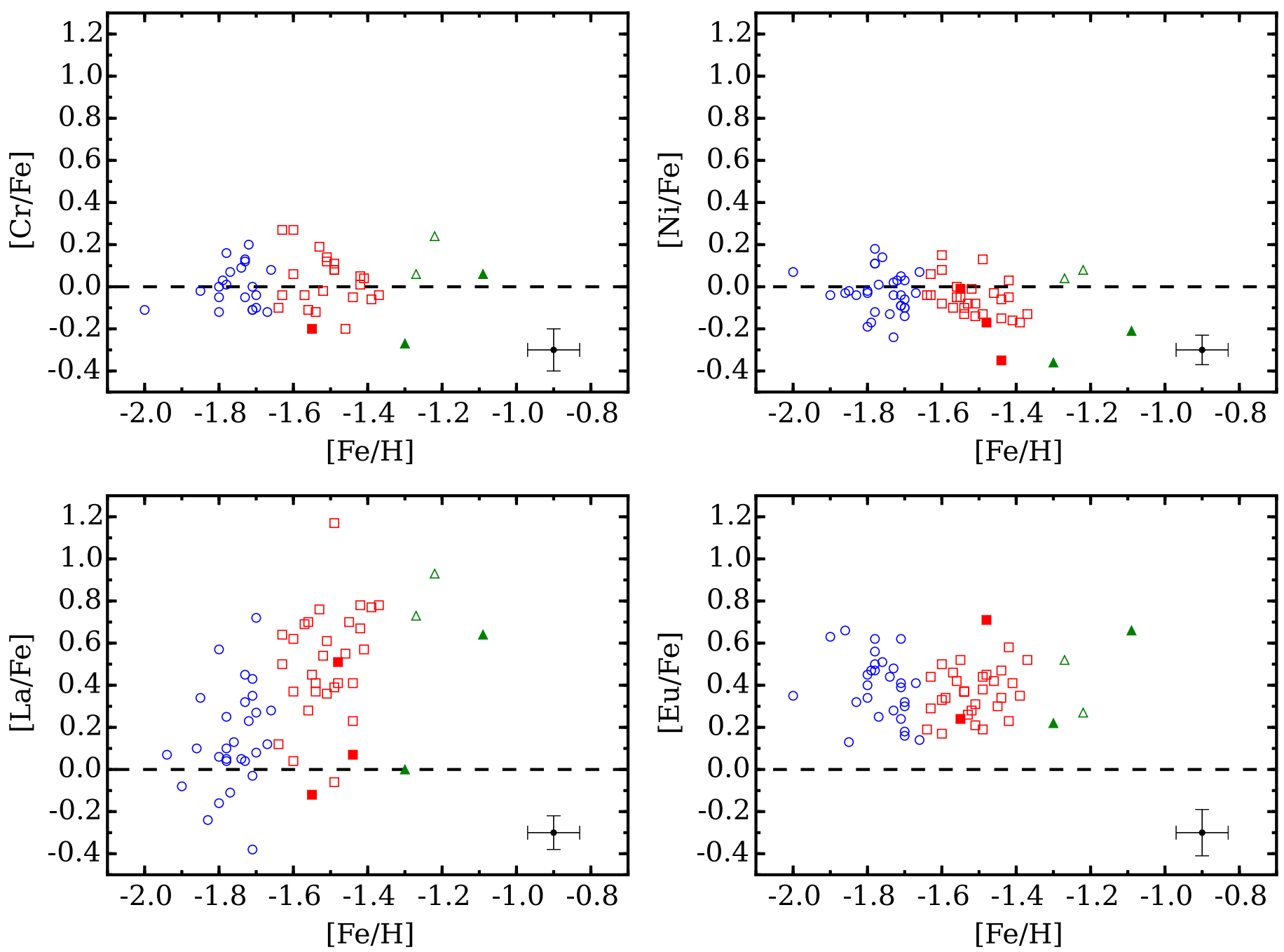

Figure 16. $[\mathrm{Cr} / \mathrm{Fe}],[\mathrm{Ni} / \mathrm{Fe}],[\mathrm{La} / \mathrm{Fe}]$, and $[\mathrm{Eu} / \mathrm{Fe}]$ abundances are plotted as a function of $[\mathrm{Fe} / \mathrm{H}]$ for all three major populations in $\mathrm{NGC} 6273$. The colors and symbols are the same as those in Figure 12.

environments, such as $\mathrm{Mn}, \mathrm{Co}, \mathrm{Zn}$, and $\mathrm{Cu}$ (e.g., Nomoto et al. 2006), may provide additional insight into whether the stars with low $[\alpha / \mathrm{Fe}],[\mathrm{Cr} / \mathrm{Fe}]$, and $[\mathrm{Ni} / \mathrm{Fe}]$ have similar origins.

\subsubsection{Neutron-capture Element Abundances}

Most of the stable isotopes heavier than the Fe-peak are produced either by the r-process over short timescales or by the s-process over much longer timescales (e.g., see the review by Sneden et al. 2008). As a result, old globular clusters tend to have heavy element compositions that are dominated by rprocess nucleosynthesis, which is evidenced by their characteristically low [La/Eu] ratios (e.g., see Gratton et al. 2004, their Figure 6). Although the Galactic globular cluster system exhibits a trend of increasing s-process contributions at higher $[\mathrm{Fe} / \mathrm{H}]$ (e.g., James et al. 2004), a small number of clusters, such as M4 (e.g., Ivans et al. 1999), deviate from this trend and exhibit significantly higher $[\mathrm{La} / \mathrm{Eu}]$ ratios. In these clusters, the gas from which their stars formed likely experienced additional, but uniform, pollution from a previous generation of 1.5-4 $M_{\odot}$ AGB stars (e.g., Busso et al. 1999).

As mentioned in Section 1, one of the "chemical tags" of iron-complex clusters is that they exhibit clear correlations between $[\mathrm{Fe} / \mathrm{H}]$ and the products of s-process enrichment. All iron-complex clusters for which the heavy elements have been measured contain populations of $\mathrm{Fe} / \mathrm{s}$-poor and $\mathrm{Fe} / \mathrm{s}$-rich stars with similar $\mathrm{Ba}$ and La enhancements (Johnson et al. 2015b; Marino et al. 2015). As a result, merger scenarios seem unlikely for every case because each cluster would have had to form from the coalescence of populations with nearly identical $\mathrm{Fe} /$ s-poor and $\mathrm{Fe} /$ s-rich compositions (but see also Gavagnin et al. 2016). Instead, we regard the combination of $[\mathrm{Fe} / \mathrm{H}]$ and s-process enhancements as a sign that iron-complex clusters were able to sustain extended star formation and selfenrichment, and that the time frame was long enough for low and intermediate mass $\mathrm{AGB}$ stars to contribute to the composition of the more metal-rich stars.

Figure 16 and Table 8 show a clear increase in [La/Fe] with $[\mathrm{Fe} / \mathrm{H}]$ for NGC 6273 , in agreement with the results of Johnson et al. (2015b). Therefore, we confirm that NGC 6273 possesses the same s-process enrichment profiles as other iron-complex clusters. We also find for $\mathrm{Eu}$ that the cluster average is about $[\mathrm{Eu} / \mathrm{Fe}]=0.4$ dex, regardless of a star's metallicity. This suggests that massive stars were largely responsible for the increase in $[\mathrm{Fe} / \mathrm{H}]$ within the cluster, and that the production rate of $\mathrm{Fe}$ and Eu was approximately constant. In Figure 17, we update the analysis of Johnson et al. (2015b) with a sample size that is $\sim 3 \times$ larger and confirm that the rise in $[\mathrm{La} / \mathrm{Fe}]$, and thus the $[\mathrm{La} / \mathrm{Eu}]$ 

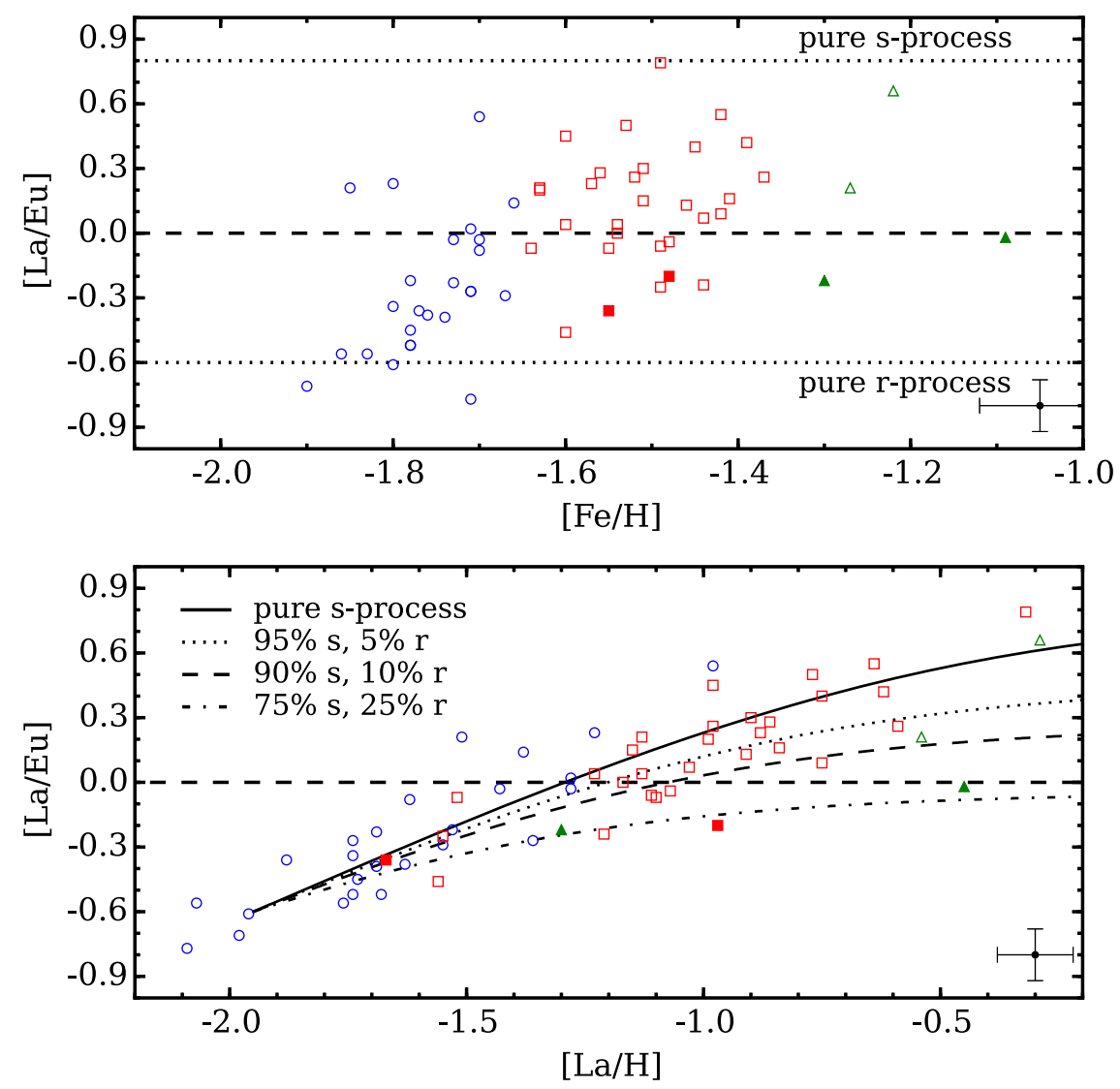

Figure 17. Top: this panel shows the correlation between $[\mathrm{La} / \mathrm{Eu}]$ and $[\mathrm{Fe} / \mathrm{H}]$ for all NGC 6273 stars observed in this work and Johnson et al. (2015b). The colors and symbols are the same as in Figure 12. The dotted lines indicate the pure r-process and pure s-process [La/Eu] abundances from Kappeler et al. (1989) and Bisterzo et al. (2010), respectively. Bottom: similar to Figure 10 in Johnson et al. (2015b) and following McWilliam et al. (2013), this panel plots [La/Eu] as a function of $[\mathrm{La} / \mathrm{H}]$. The solid black line indicates the expected change in $[\mathrm{La} / \mathrm{Eu}]$ as a function of $[\mathrm{La} / \mathrm{H}]$ when pure s-process material is added to an initial composition of pure r-process material. The dotted, dashed, and dotted-dashed dilution curves represent constant mixtures of $95 \%(\mathrm{~s}) / 5 \%(\mathrm{r}), 90 \%(\mathrm{~s}) / 10 \%(\mathrm{r})$, and $75 \%(\mathrm{~s}) / 25 \%(\mathrm{r})$ material added to an initial r-process composition. Note that the "low- $\alpha$ " stars tend to have low $[\mathrm{La} / \mathrm{Eu}]$ ratios compared to stars with similar $[\mathrm{La} / \mathrm{H}]$ or $[\mathrm{Fe} / \mathrm{H}]$.

ratio, with metallicity is due to almost pure s-process enrichment. In fact, if we assume that the most La-poor stars represent the initial pure r-process composition of the cluster, a simple dilution model shows that nearly all of the stars can be accounted for by adding $\sim 90 \%$ s-process material and $\sim 10 \%$ r-process material to the initial r-process composition. The constant r-process contribution is qualitatively in agreement with the $[\mathrm{Eu} / \mathrm{Fe}]$ observations of Figure 16 because some level of r-process enrichment is required to maintain the cluster's overall $\mathrm{Eu}$ enhancement at higher $[\mathrm{Fe} / \mathrm{H}]$.

Interestingly, the low- $\alpha$ stars in Figure 16 either have $[\mathrm{La} / \mathrm{Fe}] \sim 0.6 \mathrm{dex}$ and $[\mathrm{Eu} / \mathrm{Fe}] \sim 0.7 \mathrm{dex}$ or $[\mathrm{La} / \mathrm{Fe}] \sim 0.0 \mathrm{dex}$ and $[\mathrm{Eu} / \mathrm{Fe}] \sim 0.2$ dex. Although the origin of these stars is not clear, it is tempting to speculate that two different formation channels may exist (e.g., in situ versus accretion). We note in particular that low- $\alpha$ stars with high $[\mathrm{La} / \mathrm{Fe}]$ and $[\mathrm{Eu} / \mathrm{Fe}]$ are found in the Sagittarius field (e.g., McWilliam et al. 2013), albeit at higher $[\mathrm{Fe} / \mathrm{H}]$. The existence of these stars further strengthens the idea that at least some of the low- $\alpha$ stars in NGC 6273 could have been accreted from a surrounding field population. The low- $\alpha$ stars with lower $[\mathrm{La} / \mathrm{Fe}]$ and $[\mathrm{Eu} / \mathrm{Fe}]$ are perhaps a bigger puzzle, but they could have been formed in situ and preferentially enriched by SNe Ia or massive stars with peculiar enrichment signatures. However, Figure 17 shows that all of the low- $\alpha$ stars have about the same $[\mathrm{La} / \mathrm{Eu}]$ ratios, and may even fall on a separate enrichment sequence. In any case, the simple dilution model shown in
Figure 17 suggests that the low- $\alpha$ stars experienced significant r-process enrichment compared to a majority of the $\alpha$-enhanced metal-intermediate and metal-rich cluster stars.

\subsection{A Connection between Blue Hook Stars and Cluster Formation?}

NGC 6273 has long been known to exhibit a peculiar HB morphology that includes a very extended blue HB, a clear gap near temperatures of $\sim 20,000 \mathrm{~K}$, and a large population of blue hook stars (Piotto et al. 1999; Brown et al. 2001, 2010; Momany et al. 2004). We confirm these features with new HST color-magnitude diagrams in Figure 14, and find that NGC 6273's HB includes several distinct groups. ${ }^{24}$ Although a detailed examination of each HB group is beyond the scope of this paper, we draw attention to NGC 6273's large blue hook population in the context of its complex formation history.

Blue hook stars are among the hottest core He burning stars in old globular clusters, and are thought to form when stars reach the RGB-tip with masses low enough to delay the core He flash until after a star reaches the white dwarf cooling sequence (e.g., D’Cruz et al. 1996; Moehler et al. 2000; Brown

\footnotetext{
${ }^{24}$ We adopt the common notation that blue $\mathrm{HB}$ stars have $T_{\text {eff }} \gtrsim 8000 \mathrm{~K}$, extreme $\mathrm{HB}$ stars have $20,000 \mathrm{~K} \lesssim T_{\text {eff }} \lesssim 32,000 \mathrm{~K}$, and blue hook stars have $T_{\text {eff }} \gtrsim 32,000 \mathrm{~K}$. In Figure 14, the Grundahl jump (Grundahl et al. 1998, 1999) and Momany jump (Momany et al. 2002, 2004) are found near (F336W$\mathrm{F} 555 \mathrm{~W})_{\mathrm{o}} \sim-0.5$ and $-1.25 \mathrm{mag}$, respectively.
} 

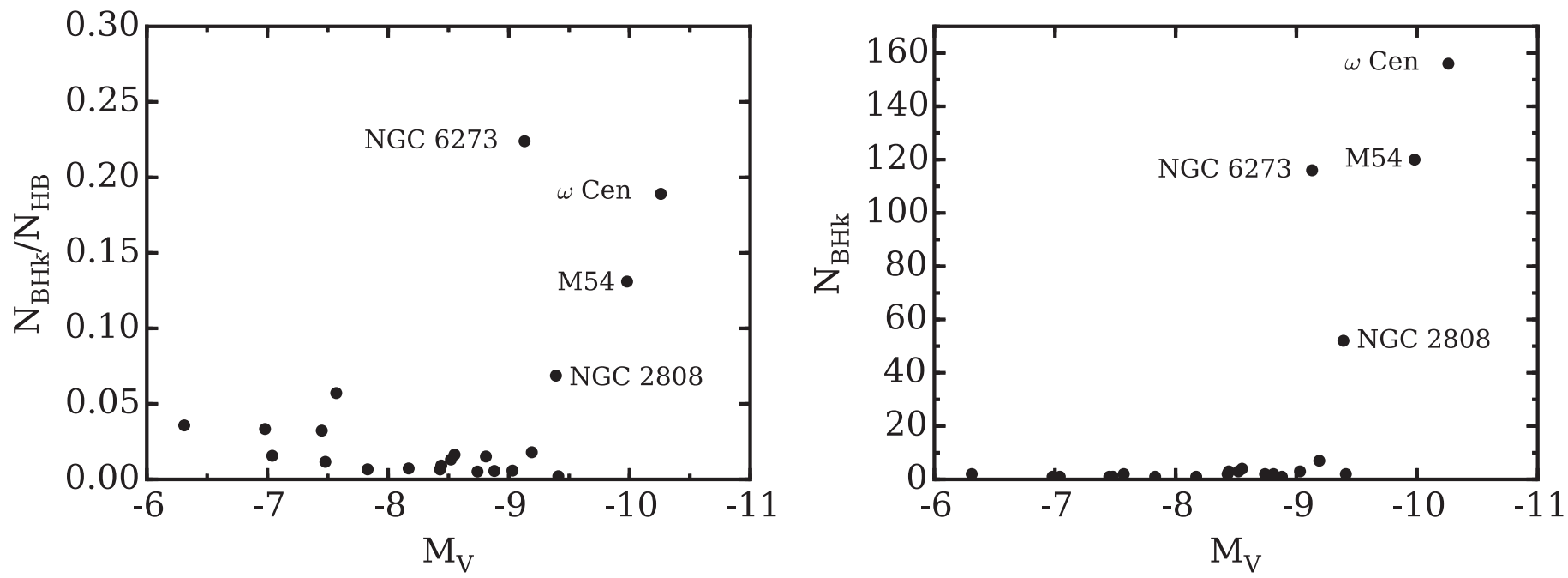

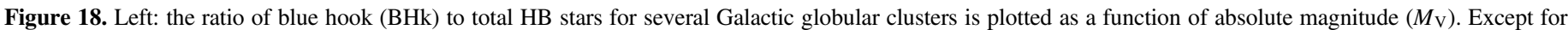

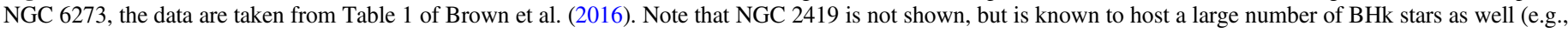

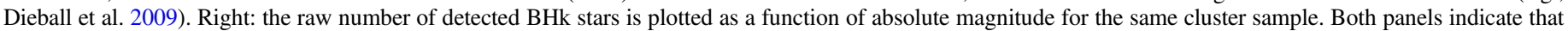
high masses and large populations of BHk stars distinguish NGC 6273, $\omega$ Cen, M54, and NGC 2808 from most clusters in the Galaxy.

et al. 2010). The presence of blue hook stars is known to correlate with cluster mass (Rosenberg et al. 2004; Dieball et al. 2009; Brown et al. 2010, 2016), which we illustrate in Figure 18 by showing that both the ratio of blue hook to canonical HB stars $\left(\frac{N_{\mathrm{BHk}}}{N_{\mathrm{HB}}}\right)$ and the raw number of blue hook stars $\left(N_{\mathrm{BHk}}\right)$ is higher in the more massive clusters. However, He enhancement is also likely tied to blue hook formation (e.g., D'Antona et al. 2002; Tailo et al. 2015).

Although present day cluster mass and the level of Heenrichment strongly correlate with the presence of blue hook stars, neither parameter nor a combination of the two parameters seems adequate to completely predict blue hook formation. For example, He-enrichment scenarios (e.g., D'Antona et al. 2010) are presently unable to explain the significant carbon enhancements that are found in He-enhanced blue hook stars (Moehler et al. 2007, 2011; Latour et al. 2014), and Figure 18 shows that clusters with similar absolute magnitudes (proxies for masses) can have vastly different blue hook populations. To illustrate this point, we note that the ironcomplex clusters NGC 6273 and M2 differ by only 0.1 mag in $M_{\mathrm{V}}$, have similarly extended blue HB morphologies, exhibit comparable light and heavy element abundance variations, have similar average metallicities and ages, and have total HB counts that agree to within $0.5 \%$, but $\mathrm{M} 2$ has a $\frac{N_{\mathrm{BHk}}}{N_{\mathrm{HB}}}$ ratio of 0.006 (3 blue hook stars) whereas NGC 6273 has $\frac{N_{\mathrm{BHk}}}{N_{\mathrm{HB}}}=0.224$ ( $\sim 120$ blue hook stars). ${ }^{25}$ Furthermore, dynamical and binary star evolutionary processes may be ruled out as explanations because the blue hook stars in clusters with large $\frac{N_{\mathrm{BHk}}}{N_{\mathrm{HB}}}$ ratios, including NGC 6273, do not exhibit radial gradients (e.g., Bedin et al. 2000; Brown et al. 2010). Therefore, additional parameters must play a role in producing blue hook stars.

Interestingly, the three objects in Figure 18 that contain $>100$ blue hook stars and have $\frac{N_{\mathrm{BHk}}}{N_{\mathrm{HB}}}>0.10$ are the ironcomplex clusters NGC 6273, M54, and $\omega$ Cen. All three clusters have about the same average metallicity, have large metallicity spreads, and exhibit extreme variations in light

\footnotetext{
${ }^{25}$ The HB and blue hook data for M2 are from Brown et al. (2016).
}

element, heavy element, and (most likely) He abundances. However, at least $\omega$ Cen and M54 are particularly noteworthy because these clusters are strongly suspected to have extragalactic origins (e.g., Bekki \& Freeman 2003; Mackey $\&$ van den Bergh 2005). The similar chemical pattern and HB morphology that NGC 6273 shares with $\omega$ Cen and M54 suggest that NGC 6273 may have also been accreted by the Milky Way. If these clusters are all remnants of dwarf galaxy systems, then it is reasonable to assume that each cluster has experienced significant mass loss. Therefore, a cluster's formation environment and initial mass may play critical roles in forming large populations of blue hook stars, and the different blue hook populations of NGC 6273 and M2 could be explained if NGC 6273 was initially much more massive than M2 and/or formed in a different environment. In this context, we note that NGC $2419^{26}$ and NGC 2808 would also be candidates that may have formed with much larger initial masses, and their higher and lower $\frac{N_{\mathrm{BHk}}}{N_{\mathrm{HB}}}$ ratios compared to NGC 6273 could be driven by their lower and higher respective metallicities. At least for NGC 2419, there are also some indications that the cluster may have an extragalactic origin (Mackey \& van den Bergh 2005).

\section{Summary}

We have measured detailed abundances, CaT metallicities, and/or radial velocities for $>800$ RGB stars ( $>300$ members) near the massive bulge globular cluster NGC 6273. The abundances and velocities are based on an analysis of highresolution $(R \approx 27,000)$ spectra obtained with the MagellanM2FS multi-fiber instrument, and includes additional metallicity and velocity measurements of $R \approx 18,000$ archival VLTFLAMES CaT spectra. The new data extend the spectroscopic work of Johnson et al. (2015b) and Yong et al. (2016) and span a broad range in luminosity and color. These data are complemented by photometric measurements of new HSTWFC3/UVIS data in the F336W, F438W, F555W, and F814W

\footnotetext{
26 NGC 2419 is omitted from Figure 18 because it was not included in the compilation by Brown et al. (2016), but likely also has $>100$ blue hook stars (Dieball et al. 2009).
} 
bands that extend from the RGB-tip down to at least 2 mag below the main-sequence turn-off.

A simple kinematic analysis indicates that $\sim 40 \%$ of our spectroscopic targets are cluster members and have heliocentric radial velocities between +120 and $+170 \mathrm{~km} \mathrm{~s}^{-1}$. We find a cluster average velocity of $+144.71 \mathrm{~km} \mathrm{~s}^{-1}$ and a dispersion of $8.57 \mathrm{~km} \mathrm{~s}^{-1}$. The cluster exhibits net rotation with a mean projected amplitude of $3.83 \mathrm{~km} \mathrm{~s}^{-1}$. A Plummer model fit to the projected radial velocity dispersion profile suggests that NGC 6273 has a central velocity dispersion of at least $10-12 \mathrm{~km} \mathrm{~s}^{-1}$ and an $A_{\text {rot. }} / \sigma_{0}$ ratio of $\sim 0.30-0.35$.

The $[\mathrm{Fe} / \mathrm{H}]$ abundances presented here follow the results of Johnson et al. (2015b), Han et al. (2015), and Yong et al. (2016) that suggest an intrinsic metallicity spread exists in NGC 6273. Using EW measurements of individual Fe I and Fe II lines, we find evidence that at least three stellar populations with different $[\mathrm{Fe} / \mathrm{H}]$ may exist: (1) a metal-poor group with $[\mathrm{Fe} / \mathrm{H}] \leqslant-1.65$; (2) a metal-intermediate group with $-1.65<[\mathrm{Fe} / \mathrm{H}] \leqslant-1.35$; and (3) a metal-rich group with $[\mathrm{Fe} / \mathrm{H}]>-1.35$. The metal-poor and metal-intermediate populations may be associated with different giant branches, and both populations may contain roughly equivalent numbers of stars. In contrast, the metal-rich population only constitutes $6 \%$ of our sample. The metal-intermediate stars may also be more centrally concentrated than the metal-poor stars, but the radial distribution differences are only observed at projected distances $\gtrsim 1$.5 from the cluster center. Similar to Yong et al. (2016), our CaT measurements extend the metal-rich tail to at least $[\mathrm{Fe} / \mathrm{H}]=-1.0$ to $-0.5 \mathrm{dex}$, but it is possible that some (or all) of these comparatively very metal-rich stars could be bulge field stars with velocities in the membership range.

The cluster's chemical abundance patterns indicate that all three major populations contain distinct sets of first (Na/Al-poor) and second $(\mathrm{Na} / \mathrm{Al}$-rich) generation stars. All three populations exhibit similar $\mathrm{Na}-\mathrm{Al}$ correlations, but the $[\mathrm{Mg} / \mathrm{Fe}]$ and $[\mathrm{Al} / \mathrm{Fe}]$ distributions suggest a complex enrichment scenario. For example, $[\mathrm{Al} / \mathrm{Fe}]$ spans about a factor of 10 in abundance for the metal-poor and metal-intermediate populations, but only the metal-intermediate stars show evidence of a $\mathrm{Mg}-\mathrm{Al}$ anticorrelation. The metal-rich stars may also exhibit a $\mathrm{Mg}-\mathrm{Al}$ anticorrelation, but the sample size is too small to draw any strong conclusions. If confirmed, a change in the $\mathrm{Mg}-\mathrm{Al}$ distribution as a function of metallicity may suggest that the gas from which the metal-intermediate and metal-rich second generation stars formed was processed at higher temperatures than the gas from which the metal-poor second generation stars formed. Notably, we did not observe any significant correlations between $\mathrm{Mg} / \mathrm{Al}$ and $\mathrm{Si}$ that would have indicated burning temperatures significantly higher than $\sim 65-70 \mathrm{MK}$, as is the case in several other massive clusters. Interestingly, the metal-intermediate and metal-rich stars with $[\mathrm{Al} / \mathrm{Fe}]>1.0$ dex have higher than expected $[\mathrm{Mg} / \mathrm{Fe}]$ abundances, which could indicate that the gas from which these stars formed was polluted by a different class or mass range of objects.

Further examination of the light element abundances indicates that the RGB and AGB stars may not have identical $[\mathrm{Na} / \mathrm{Fe}]$ and $[\mathrm{Al} / \mathrm{Fe}]$ distributions. In particular, we did not find any $\mathrm{AGB}$ stars with $[\mathrm{Na} / \mathrm{Fe}]>0.5 \mathrm{dex}$ or $[\mathrm{Al} / \mathrm{Fe}]>$ 1.0 dex. The "missing" AGB stars account for $\sim 30 \%$ of the RGB sample, which is close to the fraction of extreme HB and blue hook stars relative to the total $\mathrm{HB}$ population. We speculate that the RGB stars with the highest $[\mathrm{Na} / \mathrm{Fe}]$ and
[Al/Fe] abundances likely evolve to become extreme $\mathrm{HB}$ or blue hook stars and do not ascend the AGB.

The overall $[\alpha / \mathrm{Fe}]$ ratios may slowly decline with increasing metallicity, but most stars have $[\alpha / \mathrm{Fe}] \sim 0.3$ dex. Additionally, the Fe-peak elements exhibit solar $[\mathrm{X} / \mathrm{Fe}]$ ratios, regardless of metallicity, and the star-to-star dispersion is $\sim 0.1 \mathrm{dex}$ in all three populations. In contrast, the heavy s-process element $\mathrm{La}$ exhibits a correlated increase with metallicity that ranges from $[\mathrm{La} / \mathrm{Fe}] \sim-0.2 \mathrm{dex}$ at the lowest metallicities to $[\mathrm{La} / \mathrm{Fe}] \sim$ $0.8 \mathrm{dex}$ at the highest metallicities. However, the r-process element Eu maintains a constant abundance of $[\mathrm{Eu} / \mathrm{Fe}] \sim$ 0.4 dex across the full $[\mathrm{Fe} / \mathrm{H}]$ range. In agreement with Johnson et al. (2015b), we find that the correlated increase in $[\mathrm{La} / \mathrm{Eu}]$ with metallicity is consistent with a nearly pure sprocess enrichment pattern. Constant r-process production is required to maintain the flat $[\mathrm{Eu} / \mathrm{Fe}]$ abundance distribution, but the r-process contribution likely does not significantly exceed $\sim 10 \%$. Therefore, we confirm that NGC 6273 shares an almost identical s-process enrichment pattern with other ironcomplex clusters such as $\omega$ Cen, M2, M22, and NGC 5286.

We have also identified a population of at least five peculiar "low- $\alpha$ " stars that have $[\alpha / \mathrm{Fe}] \sim 0.0$ dex, low $[\mathrm{Na} / \mathrm{Fe}]$ and $[\mathrm{Al} / \mathrm{Fe}]$ abundances (all are first generation stars), and low $[\mathrm{La} / \mathrm{Eu}]$ ratios. Many, but not all, of the low- $\alpha$ stars also exhibit low $[\mathrm{Cr} / \mathrm{Fe}]$ and $[\mathrm{Ni} / \mathrm{Fe}]$ abundances. Although the metal-poor population does not contain any low- $\alpha$ stars, the specific frequency of low- $\alpha$ stars increases from $9 \%$ in the metal-intermediate population to $50 \%$ in the metal-rich population. However, the ratios of low- $\alpha$ stars in each population should be confirmed with future large sample observations. Interestingly, the combination of $\alpha$-enhanced and $\alpha$-poor stars in NGC 6273 closely resembles the M54 and Sagittarius field star system, and we speculate that some or all of the low- $\alpha$ stars may have been accreted from a former field population that surrounded the NGC 6273 core but had different chemistry. We note that similar populations may also be present in at least $\omega$ Cen, M2, and NGC 5286.

An examination of NGC 6273's HB revealed a particularly complex morphology. We find that the $\mathrm{HB}$ is composed of several distinct groups of stars with different masses, and that the mass range within each of the extreme $\mathrm{HB}$ and blue hook populations varies by $\lesssim 0.01 M_{\odot}$. Interestingly, the $H S T$ data show that NGC 6273 may have one of the largest blue hook populations in the Galaxy. In particular, the ratio of blue hook to canonical HB stars is $\sim 0.22$, which is a trait shared only by $\omega$ Cen, M54, NGC 2419, and NGC 2808. Since all of these clusters are very massive, and at least $\omega$ Cen, M54, and NGC 2419 are suspected to have extragalactic origins, we speculate that a cluster's initial mass and formation environment are likely critical factors in the production of blue hook stars, at least in large numbers.

This research has made use of NASA's Astrophysics Data System Bibliographic Services. This publication has made use of data products from the Two Micron All Sky Survey, which is a joint project of the University of Massachusetts and the Infrared Processing and Analysis Center/California Institute of Technology, funded by the National Aeronautics and Space Administration and the National Science Foundation. C.I.J. gratefully acknowledges support from the Clay Fellowship, administered by the Smithsonian Astrophysical Observatory. M.M. is grateful for support from the National Science Foundation to develop 
M2FS (AST-0923160) and carry out the observations reported here (AST-1312997), and to the University of Michigan for its direct support of M2FS construction and operation. M.G.W. is supported by National Science Foundation grants AST-1313045 and AST-1412999. R.M.R. acknowledges support from grant AST-1413755 from the National Science Foundation. E.W.O. acknowledges support from the National Science Foundation under grant AST-1313006. C.I.J. would like to thank David Yong for providing electronic data tables of his work, and Antonino Milone for kindly providing differential reddening measurements for this paper. Support for program \#GO-14197 was provided by NASA through a grant from the Space Telescope Science Institute, which is operated by the Association of Universities for Research in Astronomy, Inc., under NASA contract NAS 526555.

Software: IRAF, girBLDRS, DOLPHOT (Dolphin 2000), XCSAO (Kurtz \& Mink 1998), MOOG14 (Sneden 1973; 2014 version).

\section{References}

Alonso-García, J., Mateo, M., Sen, B., et al. 2012, AJ, 143, 70 Armandroff, T. E., \& Da Costa, G. S. 1991, AJ, 101, 1329

Bastian, N., Cabrera-Ziri, I., \& Salaris, M. 2015, MNRAS, 449, 3333 Bastian, N., \& Lardo, C. 2015, MNRAS, 453, 357

Battaglia, G., Irwin, M., Tolstoy, E., et al. 2008, MNRAS, 383, 183 Bedin, L. R., Piotto, G., Zoccali, M., et al. 2000, A\&A, 363, 159 Bekki, K., \& Freeman, K. C. 2003, MNRAS, 346, L11

Bellazzini, M., Bragaglia, A., Carretta, E., et al. 2012, A\&A, 538, A18 Bellazzini, M., Ibata, R. A., Chapman, S. C., et al. 2008, AJ, 136, 1147 Bellini, A., Piotto, G., Bedin, L. R., et al. 2009, A\&A, 507, 1393 Bensby, T., Yee, J. C., Feltzing, S., et al. 2013, A\&A, 549, A147 Bianchini, P., Varri, A. L., Bertin, G., \& Zocchi, A. 2013, ApJ, 772, 67 Bisterzo, S., Gallino, R., Straniero, O., Cristallo, S., \& Kappeler, F. 2010, MNRAS, 404, 1529

Bragaglia, A., Carretta, E., Gratton, R., et al. 2010a, A\&A, 519, A60 Bragaglia, A., Carretta, E., Gratton, R. G., et al. 2010b, ApJL, 720, L41 Bragaglia, A., Sneden, C., Carretta, E., et al. 2014, ApJ, 796, 68 Brown, T. M., Cassisi, S., D'Antona, F., et al. 2016, ApJ, 822, 44 Brown, T. M., Sweigart, A. V., Lanz, T., et al. 2010, ApJ, 718, 1332 Brown, T. M., Sweigart, A. V., Lanz, T., Landsman, W. B., \& Hubeny, I. 2001, ApJ, 562, 368

Busso, M., Gallino, R., \& Wasserburg, G. J. 1999, ARA\&A, 37, 239 Campbell, S. W., D’Orazi, V., Yong, D., et al. 2013, Natur, 498, 198 Carrera, R., Gallart, C., Pancino, E., \& Zinn, R. 2007, AJ, 134, 1298 Carrera, R., Pancino, E., Gallart, C., \& del Pino, A. 2013, MNRAS, 434, 1681 Carretta, E. 2014, ApJL, 795, L28

Carretta, E. 2015, ApJ, 810, 148

Carretta, E., Bragaglia, A., Gratton, R., D’Orazi, V., \& Lucatello, S. 2009a, A\&A, 508, 695

Carretta, E., Bragaglia, A., Gratton, R., \& Lucatello, S. 2009b, A\&A, 505, 139

Carretta, E., Bragaglia, A., Gratton, R. G., et al. 2007, A\&A, 464, 967

Carretta, E., Bragaglia, A., Gratton, R. G., et al. 2009c, A\&A, 505, 117

Carretta, E., Bragaglia, A., Gratton, R. G., et al. 2010a, A\&A, 520, A95

Carretta, E., Bragaglia, A., Gratton, R. G., et al. 2014, A\&A, 564, A60

Carretta, E., D'Orazi, V., Gratton, R. G., \& Lucatello, S. 2012, A\&A, 543, A117

Carretta, E., Gratton, R. G., Bragaglia, A., et al. 2013, ApJ, 769, 40

Carretta, E., Gratton, R. G., Lucatello, S., et al. 2010b, ApJL, 722, L1

Carretta, E., Lucatello, S., Gratton, R. G., Bragaglia, A., \& D’Orazi, V. 2011, A\&A, 533, A69

Castelli, F., \& Kurucz, R. L. 2004, arXiv:astro-ph/0405087

Chen, C. W., \& Chen, W. P. 2010, ApJ, 721, 1790

Cohen, J. G. 1978, ApJ, 223, 487

Cohen, J. G. 2004, AJ, 127, 1545

Cohen, J. G., \& Kirby, E. N. 2012, ApJ, 760, 86

Cohen, J. G., \& Meléndez, J. 2005, AJ, 129, 303

Cole, A. A., Smecker-Hane, T. A., Tolstoy, E., Bosler, T. L., \& Gallagher, J. S. 2004, MNRAS, 347, 367

Cordero, M. J., Pilachowski, C. A., Johnson, C. I., et al. 2014, ApJ, 780, 94
Côté, P., Welch, D. L., Fischer, P., \& Gebhardt, K. 1995, ApJ, 454, 788 Cottrell, P. L., \& Da Costa, G. S. 1981, ApJL, 245, L79

Da Costa, G. S. 2016a, in IAU Symp. 317, The General Assembly of Galaxy Halos: Structure, Origin and Evolution, ed. A. Bragaglia et al. (Cambridge: Cambridge Univ. Press), 110

Da Costa, G. S. 2016b, MNRAS, 455, 199

Da Costa, G. S., Held, E. V., Saviane, I., \& Gullieuszik, M. 2009, ApJ, 705,1481

Da Costa, G. S., Norris, J. E., \& Yong, D. 2013, ApJ, 769, 8

D'Antona, F., Caloi, V., Montalbán, J., Ventura, P., \& Gratton, R. 2002, A\&A, 395, 69

D'Antona, F., Caloi, V., \& Ventura, P. 2010, MNRAS, 405, 2295

D'Antona, F., Vesperini, E., D'Ercole, A., et al. 2016, MNRAS, 458, 2122

Davidge, T. J. 2000, AJ, 120, 1853

D’Cruz, N. L., Dorman, B., Rood, R. T., \& O’Connell, R. W. 1996, ApJ, 466, 359

Dieball, A., Knigge, C., Maccarone, T. J., et al. 2009, MNRAS, 394, L56

Dolphin, A. E. 2000, PASP, 112, 1383

D'Orazi, V., Gratton, R., Lucatello, S., et al. 2010, ApJL, 719, L213

Dotter, A., Chaboyer, B., Jevremović, D., et al. 2008, ApJS, 178, 89

Dupree, A. K., Avrett, E. H., \& Kurucz, R. L. 2016, ApJL, 821, L7

Dupree, A. K., Strader, J., \& Smith, G. H. 2011, ApJ, 728, 155

Ferraro, F. R., Dalessandro, E., Mucciarelli, A., et al. 2009, Natur, 462, 483

García-Hernández, D. A., Mészáros, S., Monelli, M., et al. 2015, ApJL, 815, L4

Gavagnin, E., Mapelli, M., \& Lake, G. 2016, MNRAS, 461, 1276

Girardi, L., Dalcanton, J., Williams, B., et al. 2008, PASP, 120, 583

Gosling, A. J., Bandyopadhyay, R. M., \& Blundell, K. M. 2009, MNRAS, 394, 2247

Gratton, R., Sneden, C., \& Carretta, E. 2004, ARA\&A, 42, 385

Gratton, R. G., Bonifacio, P., Bragaglia, A., et al. 2001, A\&A, 369, 87

Gratton, R. G., D’Orazi, V., Bragaglia, A., Carretta, E., \& Lucatello, S. 2010 , A\&A, 522, A77

Greggio, L., \& Renzini, A. 1990, ApJ, 364, 35

Grillmair, C. J., Freeman, K. C., Irwin, M., \& Quinn, P. J. 1995, AJ, 109, 2553

Grundahl, F., Catelan, M., Landsman, W. B., Stetson, P. B., \& Andersen, M. I. 1999, ApJ, 524, 242

Grundahl, F., VandenBerg, D. A., \& Andersen, M. I. 1998, ApJL, 500, L179

Han, S.-I., Lim, D., Seo, H., \& Lee, Y.-W. 2015, ApJL, 813, L43

Harris, W. E. 1996, AJ, 112, 1487

Harris, W. E., Racine, R., \& de Roux, J. 1976, ApJS, 31, 13

Idiart, T. P., Thevenin, F., \& de Freitas Pacheco, J. A. 1997, AJ, 113, 1066

Ivans, I. I., Kraft, R. P., Sneden, C., et al. 2001, AJ, 122, 1438

Ivans, I. I., Sneden, C., Kraft, R. P., et al. 1999, AJ, 118, 1273

James, G., François, P., Bonifacio, P., et al. 2004, A\&A, 427, 825

Johnson, C. I., McDonald, I., Pilachowski, C. A., et al. 2015a, AJ, 149, 71

Johnson, C. I., \& Pilachowski, C. A. 2010, ApJ, 722, 1373

Johnson, C. I., \& Pilachowski, C. A. 2012, ApJL, 754, L38

Johnson, C. I., Rich, R. M., Kobayashi, C., et al. 2013, ApJ, 765, 157

Johnson, C. I., Rich, R. M., Pilachowski, C. A., et al. 2015b, AJ, 150, 63

Kacharov, N., Bianchini, P., Koch, A., et al. 2014, A\&A, 567, A69

Kappeler, F., Beer, H., \& Wisshak, K. 1989, RPPh, 52, 945

Kimmig, B., Seth, A., Ivans, I. I., et al. 2015, AJ, 149, 53

Kraft, R. P., Sneden, C., Smith, G. H., et al. 1997, AJ, 113, 279

Kunder, A., Koch, A., Rich, R. M., et al. 2012, AJ, 143, 57

Kurtz, M. J., \& Mink, D. J. 1998, PASP, 110, 934

Kuzma, P. B., Da Costa, G. S., Mackey, A. D., \& Roderick, T. A. 2016, MNRAS, 461, 3639

Lane, R. R., Kiss, L. L., Lewis, G. F., et al. 2009, MNRAS, 400, 917

Lane, R. R., Kiss, L. L., Lewis, G. F., et al. 2010a, MNRAS, 401, 2521

Lane, R. R., Kiss, L. L., Lewis, G. F., et al. 2010b, MNRAS, 406, 2732

Langer, G. E., Hoffman, R., \& Sneden, C. 1993, PASP, 105, 301

Langer, G. E., Hoffman, R. E., \& Zaidins, C. S. 1997, PASP, 109, 244

Lapenna, E., Lardo, C., Mucciarelli, A., et al. 2016, ApJL, 826, L1

Lapenna, E., Mucciarelli, A., Lanzoni, B., et al. 2014, ApJ, 797, 124

Lardo, C., Bellazzini, M., Pancino, E., et al. 2011, A\&A, 525, A114

Lardo, C., Mucciarelli, A., \& Bastian, N. 2016, MNRAS, 457, 51

Lardo, C., Pancino, E., Bellazzini, M., et al. 2015, A\&A, 573, A115

Latour, M., Randall, S. K., Fontaine, G., et al. 2014, ApJ, 795, 106

Law, D. R., \& Majewski, S. R. 2010, ApJ, 718, 1128

Lawler, J. E., Wickliffe, M. E., den Hartog, E. A., \& Sneden, C. 2001, ApJ, 563,1075

Lee, J.-W. 2015, ApJS, 219, 7

Lee, J.-W. 2016, ApJS, 226, 16

Lim, D., Han, S.-I., Lee, Y.-W., et al. 2015, ApJS, 216, 19 
Lind, K., Bergemann, M., \& Asplund, M. 2012, MNRAS, 427, 50 Mackey, A. D., \& van den Bergh, S. 2005, MNRAS, 360, 631

MacLean, B. T., Campbell, S. W., De Silva, G. M., et al. 2016, MNRAS, 460, L69

Mallia, E. A. 1978, A\&A, 70, 115

Marino, A. F., Milone, A. P., Casagrande, L., et al. 2016, MNRAS, 459, 610

Marino, A. F., Milone, A. P., Karakas, A. I., et al. 2015, MNRAS, 450, 815

Marino, A. F., Milone, A. P., Piotto, G., et al. 2009, A\&A, 505, 1099

Marino, A. F., Milone, A. P., Piotto, G., et al. 2011a, ApJ, 731, 64

Marino, A. F., Milone, A. P., Przybilla, N., et al. 2014a, MNRAS, 437, 1609

Marino, A. F., Milone, A. P., Yong, D., et al. 2014b, MNRAS, 442, 3044

Marino, A. F., Sneden, C., Kraft, R. P., et al. 2011b, A\&A, 532, A8

Massari, D., Mucciarelli, A., Ferraro, F. R., et al. 2014, ApJ, 795, 22

Mateo, M., Bailey, J. I., Crane, J., et al. 2012, Proc. SPIE, 8446, 84464Y

Mauro, F., Moni Bidin, C., Geisler, D., et al. 2014, A\&A, 563, A76

McWilliam, A. 1997, ARA\&A, 35, 503

McWilliam, A., Wallerstein, G., \& Mottini, M. 2013, ApJ, 778, 149

Mészáros, S., Martell, S. L., Shetrone, M., et al. 2015, AJ, 149, 153

Milone, A. P., Marino, A. F., Piotto, G., et al. 2013, ApJ, 767, 120

Milone, A. P., Marino, A. F., Piotto, G., et al. 2015a, MNRAS, 447, 927

Milone, A. P., Marino, A. F., Piotto, G., et al. 2015b, ApJ, 808, 51

Milone, A. P., Piotto, G., Bedin, L. R., et al. 2012, A\&A, 540, A16

Moehler, S., Dreizler, S., Lanz, T., et al. 2007, A\&A, 475, L5

Moehler, S., Dreizler, S., Lanz, T., et al. 2011, A\&A, 526, A136

Moehler, S., Sweigart, A. V., Landsman, W. B., \& Heber, U. 2000, A\&A, 360,120

Momany, Y., Bedin, L. R., Cassisi, S., et al. 2004, A\&A, 420, 605

Momany, Y., Piotto, G., Recio-Blanco, A., et al. 2002, ApJL, 576, L65

Mucciarelli, A., Bellazzini, M., Ibata, R., et al. 2012, MNRAS, 426, 2889

Mucciarelli, A., Bellazzini, M., Merle, T., et al. 2015a, ApJ, 801, 68

Mucciarelli, A., Carretta, E., Origlia, L., \& Ferraro, F. R. 2008, AJ, 136, 375

Mucciarelli, A., Lapenna, E., Massari, D., et al. 2015b, ApJ, 809, 128

Mucciarelli, A., Lapenna, E., Massari, D., Ferraro, F. R., \& Lanzoni, B. 2015c, ApJ, 801, 69

Mucciarelli, A., Lovisi, L., Lanzoni, B., \& Ferraro, F. R. 2014, ApJ, 786, 14

Nataf, D. M., Gonzalez, O. A., Casagrande, L., et al. 2016, MNRAS, 456, 2692

Nataf, D. M., Gould, A., Fouqué, P., et al. 2013, ApJ, 769, 88

Navin, C. A., Martell, S. L., \& Zucker, D. B. 2015, MNRAS, 453, 531

Navin, C. A., Martell, S. L., \& Zucker, D. B. 2016, arXiv:1606.06430

Ness, M., Freeman, K., Athanassoula, E., et al. 2013a, MNRAS, 432, 2092

Ness, M., Freeman, K., Athanassoula, E., et al. 2013b, MNRAS, 430, 836

Nomoto, K., Tominaga, N., Umeda, H., Kobayashi, C., \& Maeda, K. 2006, $\mathrm{NuPhA}, 777,424$

Norris, J., Cottrell, P. L., Freeman, K. C., \& Da Costa, G. S. 1981, ApJ, 244,205

Norris, J. E., \& Da Costa, G. S. 1995, ApJ, 447, 680

Norris, J. E., Freeman, K. C., \& Mighell, K. J. 1996, ApJ, 462, 241

Olszewski, E. W., Saha, A., Knezek, P., et al. 2009, AJ, 138, 1570

Olszewski, E. W., Schommer, R. A., Suntzeff, N. B., \& Harris, H. C. 1991, AJ, 101,515

Origlia, L., Ferraro, F. R., Bellazzini, M., \& Pancino, E. 2003, ApJ, 591, 916

Origlia, L., Massari, D., Rich, R. M., et al. 2013, ApJL, 779, L5

Origlia, L., Rich, R. M., Ferraro, F. R., et al. 2011, ApJL, 726, L20

Pancino, E., Pasquini, L., Hill, V., Ferraro, F. R., \& Bellazzini, M. 2002, ApJL, 568, L101

Pasquini, L., Mauas, P., Käufl, H. U., \& Cacciari, C. 2011, A\&A, 531, A35

Peterson, R. C. 1980, ApJL, 237, L87

Pietrinferni, A., Cassisi, S., Salaris, M., \& Castelli, F. 2006, ApJ, 642, 797

Pilachowski, C., Leep, E. M., Wallerstein, G., \& Peterson, R. C. 1982, ApJ, 263,187

Pilachowski, C. A., Sneden, C., \& Kraft, R. P. 1996a, AJ, 111, 1689
Pilachowski, C. A., Sneden, C., Kraft, R. P., \& Langer, G. E. 1996b, AJ, 112,545

Piotto, G., Bedin, L. R., Anderson, J., et al. 2007, ApJL, 661, L53

Piotto, G., Milone, A. P., Bedin, L. R., et al. 2015, AJ, 149, 91

Piotto, G., Zoccali, M., King, I. R., et al. 1999, AJ, 118, 1727

Plummer, H. C. 1911, MNRAS, 71, 460

Prantzos, N., Charbonnel, C., \& Iliadis, C. 2007, A\&A, 470, 179

Pritzl, B. J., Venn, K. A., \& Irwin, M. 2005, AJ, 130, 2140

Racine, R. 1973, AJ, 78, 180

Renzini, A., D’Antona, F., Cassisi, S., et al. 2015, MNRAS, 454, 4197

Rey, S.-C., Lee, Y.-W., Ree, C. H., et al. 2004, AJ, 127, 958

Roederer, I. U. 2011, ApJL, 732, L17

Roederer, I. U., \& Thompson, I. B. 2015, MNRAS, 449, 3889

Rosenberg, A., Recio-Blanco, A., \& García-Marín, M. 2004, ApJ, 603, 135

Rutledge, G. A., Hesser, J. E., Stetson, P. B., et al. 1997, PASP, 109, 883

Salinas, R., \& Strader, J. 2015, ApJ, 809, 169

Saviane, I., da Costa, G. S., Held, E. V., et al. 2012, A\&A, 540, A27

Shetrone, M. D., \& Keane, M. J. 2000, AJ, 119, 840

Simmerer, J., Sneden, C., Ivans, I. I., et al. 2003, AJ, 125, 2018

Skrutskie, M. F., Cutri, R. M., Stiening, R., et al. 2006, AJ, 131, 1163

Smith, G. H., \& Norris, J. E. 1993, AJ, 105, 173

Smith, V. V., Suntzeff, N. B., Cunha, K., et al. 2000, AJ, 119, 1239

Sneden, C. 1973, ApJ, 184, 839

Sneden, C., Cowan, J. J., \& Gallino, R. 2008, ARA\&A, 46, 241

Sneden, C., Ivans, I. I., \& Kraft, R. P. 2000, MmSAI, 71, 657

Sneden, C., Kraft, R. P., Guhathakurta, P., Peterson, R. C., \& Fulbright, J. P. 2004, AJ, 127, 2162

Sneden, C., Kraft, R. P., Prosser, C. F., \& Langer, G. E. 1991, AJ, 102, 2001

Sneden, C., Lucatello, S., Ram, R. S., Brooke, J. S. A., \& Bernath, P. 2014, ApJS, 214, 26

Sosin, C., Dorman, B., Djorgovski, S. G., et al. 1997, ApJL, 480, L35

Starkenburg, E., Hill, V., Tolstoy, E., et al. 2010, A\&A, 513, A34

Suntzeff, N. B. 1981, ApJS, 47, 1

Suntzeff, N. B., \& Kraft, R. P. 1996, AJ, 111, 1913

Tailo, M., D’Antona, F., Vesperini, E., et al. 2015, Natur, 523, 318

Timmes, F. X., Woosley, S. E., \& Weaver, T. A. 1995, ApJS, 98, 617

Tinsley, B. M. 1979, ApJ, 229, 1046

Udalski, A. 2003, ApJ, 590, 284

Valcarce, A. A. R., \& Catelan, M. 2011, A\&A, 533, A120

Valenti, E., Ferraro, F. R., \& Origlia, L. 2007, AJ, 133, 1287

Vásquez, S., Zoccali, M., Hill, V., et al. 2015, A\&A, 580, A121

Ventura, P., D'Antona, F., Di Criscienzo, M., et al. 2012, ApJL, 761, L30

Vesperini, E., McMillan, S. L. W., D’Antona, F., \& D’Ercole, A. 2013, MNRAS, 429, 1913

Villanova, S., Geisler, D., Carraro, G., Moni Bidin, C., \& Muñoz, C. 2013, ApJ, 778, 186

Villanova, S., Geisler, D., Piotto, G., \& Gratton, R. G. 2012, ApJ, 748, 62

Walker, A. R., Kunder, A. M., Andreuzzi, G., et al. 2011, MNRAS, 415, 643

Wang, Y., Primas, F., Charbonnel, C., et al. 2016, A\&A, 592, A66

White, R. E., \& Shawl, S. J. 1987, ApJ, 317, 246

Williams, B. F., Lang, D., Dalcanton, J. J., et al. 2014, ApJS, 215, 9

Woosley, S. E., \& Weaver, T. A. 1995, ApJS, 101, 181

Worley, C. C., \& Cottrell, P. L. 2010, MNRAS, 406, 2504

Yong, D., Da Costa, G. S., \& Norris, J. E. 2016, MNRAS, 460, 1846

Yong, D., \& Grundahl, F. 2008, ApJL, 672, L29

Yong, D., Grundahl, F., Nissen, P. E., Jensen, H. R., \& Lambert, D. L. 2005, A\&A, 438, 875

Yong, D., Meléndez, J., Grundahl, F., et al. 2013, MNRAS, 434, 3542

Yong, D., Roederer, I. U., Grundahl, F., et al. 2014, MNRAS, 441, 3396

Zoccali, M., Gonzalez, O. A., Vasquez, S., et al. 2014, A\&A, 562, A66

Zoccali, M., Hill, V., Lecureur, A., et al. 2008, A\&A, 486, 177 